\title{
GWTC-2: Compact Binary Coalescences Observed by LIGO and Virgo during the First Half of the Third Observing Run
}

\author{
R. Abbott et al. \\ (LIGO Scientific Collaboration and Virgo Collaboration)
} (Received 30 October 2020; revised 23 February 2021; accepted 20 April 2021; published 9 June 2021;
corrected 1 September 2021 and 27 April 2022)

\begin{abstract}
We report on gravitational-wave discoveries from compact binary coalescences detected by Advanced LIGO and Advanced Virgo in the first half of the third observing run (O3a) between 1 April 2019 15:00 UTC and 1 October 2019 15:00 UTC. By imposing a false-alarm-rate threshold of two per year in each of the four search pipelines that constitute our search, we present 39 candidate gravitational-wave events. At this threshold, we expect a contamination fraction of less than 10\%. Of these, 26 candidate events were reported previously in near-real time through gamma-ray coordinates network notices and circulars; 13 are reported here for the first time. The catalog contains events whose sources are black hole binary mergers up to a redshift of approximately 0.8 , as well as events whose components cannot be unambiguously identified as black holes or neutron stars. For the latter group, we are unable to determine the nature based on estimates of the component masses and spins from gravitational-wave data alone. The range of candidate event masses which are unambiguously identified as binary black holes (both objects $\geq 3 M_{\odot}$ ) is increased compared to GWTC-1, with total masses from approximately $14 M_{\odot}$ for GW190924_021846 to approximately $150 M_{\odot}$ for GW190521. For the first time, this catalog includes binary systems with significantly asymmetric mass ratios, which had not been observed in data taken before April 2019. We also find that 11 of the 39 events detected since April 2019 have positive effective inspiral spins under our default prior (at $90 \%$ credibility), while none exhibit negative effective inspiral spin. Given the increased sensitivity of Advanced LIGO and Advanced Virgo, the detection of 39 candidate events in approximately 26 weeks of data (approximately 1.5 per week) is consistent with GWTC-1.
\end{abstract}

DOI: 10.1103/PhysRevX.11.021053

\section{INTRODUCTION}

Since the discovery of gravitational waves from a binary black hole (BBH) coalescence in 2015 [1], the Advanced LIGO [2] and Advanced Virgo [3] gravitational-wave detectors have opened a new window on our Universe [4-8]. Binary black hole observations allow us to probe gravity in the strong-field regime $[9,10]$ and to establish the rate and population properties of $\mathrm{BBH}$ coalescences [11]. In addition to BBHs, Advanced LIGO and Advanced Virgo detected the first gravitational-wave signal from a binary neutron star (BNS) coalescence, GW170817 [12], which is also the first joint detection of gravitational waves and electromagnetic emission [13,14]. Gravitational-wave discoveries have a profound impact on physics, astronomy, and astrophysics [13,15-19], and the public release of LIGO and Virgo data $[20,21]$ enables groups other than the

${ }^{*}$ Full author list given at the end of the article.

Published by the American Physical Society under the terms of the Creative Commons Attribution 4.0 International license. Further distribution of this work must maintain attribution to the author(s) and the published article's title, journal citation, and DOI.
Subject Areas: Gravitation

LIGO Scientific Collaboration and Virgo Collaboration (LVC) to perform analyses searching for gravitational-wave signals [22-28] and to report additional candidate events in some cases.

We present the results of searches for compact binaries in Advanced LIGO and Advanced Virgo data taken between 1 April 2019 15:00 UTC and 1 October 2019 15:00 UTC. This period, referred to as O3a, is the first six months of Advanced LIGO and Advanced Virgo's 11-month-long third observing run. The first gravitational-wave transient catalog (GWTC-1) of compact binary coalescences (CBCs) includes candidate events observed by Advanced LIGO and Advanced Virgo during the first $(\mathrm{O} 1)$ and second $(\mathrm{O} 2)$ observing runs [8]. The increased sensitivity of Advanced LIGO and Advanced Virgo during O3a enables us to increase the number of confident gravitational-wave detections more than threefold over GWTC-1. Together, GWTC-1 and the new candidate events presented here comprise GWTC-2. Figure 1 shows this consistent increase in both the effective binary neutron star volume time (BNS $\mathrm{VT}$ ) of the gravitational-wave network and the number of detections across these observing runs. The BNS VT is a Euclidean sensitive volume of the detector network [29,30] multiplied by the live time of the network and should be approximately proportional to the total number of 


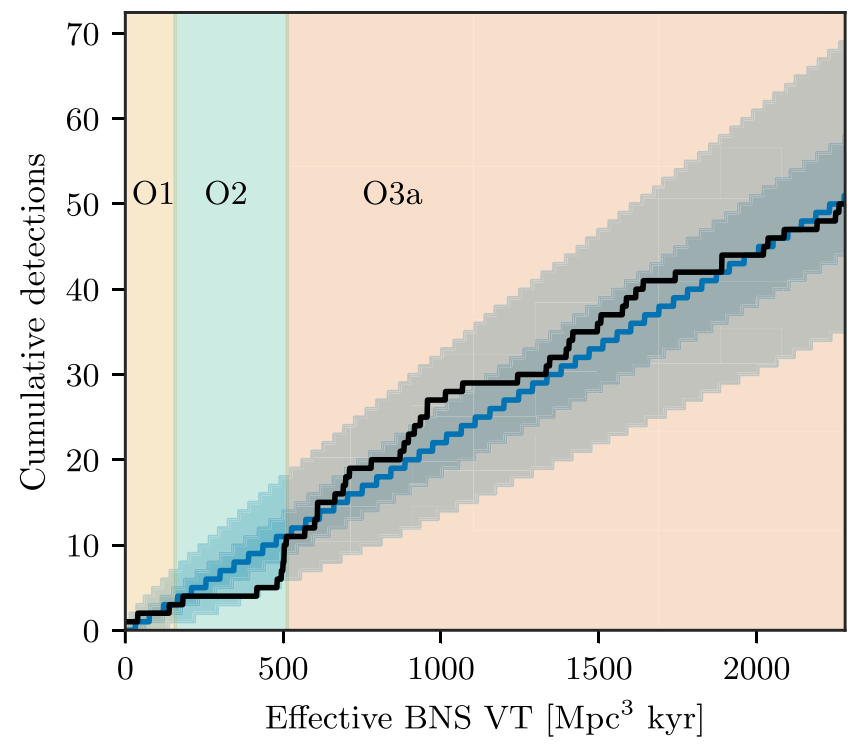

FIG. 1. The number of compact binary coalescence detections versus the effective VT to which the gravitational-wave network is sensitive to BNS coalescences. The effective VT is defined as the Euclidean sensitive volume [30] of the second-most-sensitive detector in the network at a given time, multiplied by the live time of that network configuration. The Euclidean sensitive volume of the network is the volume of a sphere with a radius given by the BNS inspiral range [29,30] (shown in Fig. 3) of the second-mostsensitive detector in the network. To account for the addition of single-detector candidates in O3a, a single-detector Euclidean sensitive volume is also included, defined using the inspiral range of the most sensitive detector divided by 1.5 . The effective BNS VT does not account for differences in sensitivity across the entire population of signals detected, necessary cosmological corrections, or changes to analysis pipeline efficiency between observing runs but, as shown in this figure, is consistent with the currently observed rate of detections. The colored bands indicate the three runs: $\mathrm{O} 1, \mathrm{O} 2$, and O3a. The black line is the cumulative number of confident detections of all compact binary coalescences (including black holes and neutron stars) for GWTC-1 [8] and this catalog. The blue line, dark blue band, and light blue band are the median, $50 \%$ confidence interval, and $90 \%$ confidence interval, respectively, of draws from a Poisson fit to the number of detections at the end of O3a. The increase in detection rate is dominated by improvements to the sensitivity of the LIGO and Virgo detectors with changes in analysis methods between observing runs being subdominant.

detections. Our analysis of the $\mathrm{O} 3 \mathrm{a}$ dataset results in 39 gravitational-wave candidate events passing our false alarm rate (FAR) threshold of 2.0 per year. Given our use of multiple search pipelines to identify candidate events, we expect approximately three false alarms, i.e., candidate events caused by instrumental noise, to be present in this catalog. It is not possible to determine with certainty which specific candidate events are due to noise; instead, we provide statistical measures of false alarm rate and probability of astrophysical origin. Among these candidate events, 26 have been reported previously in real-time processing via GCN notices and circulars [31]. Furthermore, four gravitational-wave candidate events from $\mathrm{O} 3 \mathrm{a}$ are already published separately due to their interesting properties: GW190425 [32] is the second gravitational-wave event consistent with a BNS coalescence; GW190412 [32] is the first BBH observation with definitively asymmetric component masses, which also produced detectable gravitational radiation beyond the leading quadrupolar order; GW190814 [33] is an even more asymmetric system having an approximately $23 M_{\odot}$ object merging with an approximately $2.6 M_{\odot}$ object, making the latter either the lightest black hole or heaviest neutron star known to be in a double compact object system; GW190521 [34,35] is a BBH with total mass of approximately $150 M_{\odot}$ having a primary mass above $65 M_{\odot}$ at $99 \%$ credibility.

Here, we present 13 candidate events for the first time along with the 26 previously reported candidates. Among the 39 candidates, we find gravitational-wave emission consistent with the coalescence of BBHs, BNSs, and neutron star-black hole binaries (NSBHs).

We report on the status of the Advanced LIGO and Advanced Virgo gravitational-wave detectors (Sec. II) and the properties and quality of the data taken during the analyzed period (Sec. III). We then describe the analysis methods that lead to the identification of the 39 gravitational-wave candidates (Sec. IV), as well as the inference of their parameters (Sec. V). Next, we report the significance of the identified candidates, as well as a comparison to the public gravitational-wave alerts (Sec. VI). Finally, we discuss the properties (Sec. VII) and the reconstructed waveforms (Sec. VIII) of each event. Further interpretation of the binary population is conducted in companion papers [36,37]. We will analyze the second half of Advanced LIGO and Advanced Virgo's third observing run (O3b) in future publications.

\section{INSTRUMENTS}

The Advanced LIGO [2] and Advanced Virgo [3] detectors are kilometer-scale laser interferometers [38]. The current generation of detectors started operations in 2015 and since then have been alternating periods of observation with periods of tuning and improvement. Since O1 [39] and O2 [8], the sensitivity and robustness of the detectors improved significantly.

The LIGO detectors underwent several upgrades between the end of $\mathrm{O} 2$ and the start of O3a [40]. The main laser sources were replaced to allow for higher operating powers. The LIGO Hanford detector operate with $37 \mathrm{~W}$ of input power and the Livingston detector with $40 \mathrm{~W}$. Those levels can be compared to 30 and $25 \mathrm{~W}$ during $\mathrm{O} 2$ for Hanford and Livingston, respectively. The laser sources replacement also reduces fluctuations in the input beam pointing and size that were previously detrimental for the detector sensitivity [41]. At both LIGO detectors, the 
two end test masses were replaced with mirrors with lower scattering losses [42], allowing for higher circulating power. Additionally, annular test masses were installed to reduce noise induced by residual gas damping [43]. In the Hanford interferometer, one of the two input test masses was also replaced, because the one which was previously installed had a large point absorber [44] that limited the amount of power that could be handled in the arm cavities.

The buildup of electric charge on the test masses was also an issue during previous runs; therefore, several actions were undertaken to mitigate the contribution of this noise source: Electric field meters were installed in end stations to monitor the local electric field; baffles were installed in front of the vacuum system ion pumps to mitigate charging, and a test mass discharging system was put in operation and successfully deployed on all LIGO Hanford test masses.

Parametric instabilities [45], i.e., radiation-pressureinduced excitation of the test masses' mechanical modes, also limit the maximum power allowed into the interferometer. This problem is mitigated with the installation of acoustic mode dampers [46] that reduce the mechanical quality factor of the test mass resonant modes and, thus, suppress parametric instabilities.

The high-frequency $(\gtrsim 1 \mathrm{kHz})$ sensitivity of both detectors is significantly improved compared to the $\mathrm{O} 2$ observing run (a factor of 1.68 for the Hanford detector and 1.96 for the Livingston detector), partially due to the increased circulating power made possible by the improvements already discussed and by the installation of squeezed light sources $[47,48]$ to reduce the quantum vacuum noise entering the interferometers [49], making O3 the first observing run of the LIGO detectors that routinely implements quantum noise-reduction techniques. GEO600 has been using the same approach since 2011 [50,51] but has not detected gravitational waves so far due to an overall too-low sensitivity.

Additionally, many beam dumps and baffles were installed at both LIGO sites, to mitigate the effect of scattered light [52] that can be the source of nonstationary disturbances. Finally, the feedback control systems for the seismic isolation and for the angular and longitudinal control of the instruments were improved, increasing the detectors' duty cycle and robustness against external disturbances. With respect to O2, the LIGO Hanford median BNS inspiral range, as defined in Ref. [29], increased by a factor 1.64 (from 66 to $108 \mathrm{Mpc}$ ) and the LIGO Livingston median range by 1.53 (from 88 to $135 \mathrm{Mpc}$ ).

Also, in Virgo between the $\mathrm{O} 2$ and the $\mathrm{O} 3 \mathrm{a}$ observing runs, many upgrades have been implemented to boost the sensitivity. The most important upgrade is the replacement of the steel wires suspending the four test masses with fused-silica fibers [53] to improve the sensitivity below $100 \mathrm{~Hz}$. This upgrade was achieved by changing the design of the final stage of the mirror suspension to improve the screening of the fused-silica fibers [54] from residual particles injected by the vacuum system. In parallel, the vacuum system was modified to avoid dust pollution of the environment.

Another upgrade to improve the low-frequency sensitivity is the suspension of the external injection bench (used to manipulate and steer the input laser beam into the interferometer). In this way, the seismic motion of the optics is reduced and, consequently, the beam jitter noise contribution [55,56].

The major upgrade to boost the Virgo high-frequency sensitivity is the installation of a more powerful laser that can deliver more than $65 \mathrm{~W}$ output power. After some commissioning activities at different power values, the laser power injected into the interferometer is set to $19 \mathrm{~W}$, improving both the sensitivity and the stability of the interferometer at the same time. The laser power is almost doubled compared to the $10 \mathrm{~W}$ injected during $\mathrm{O} 2$ [57]. In this configuration, due to the marginally stable power-recycling cavity, the aberration induced by thermal effects prevents a reliable and robust interferometer longitudinal control and worsens the alignment performances. Therefore, the thermal compensation system actuators were used to stabilize the powerrecycling cavity. The end test masses' radii of curvature were tuned with the ring heaters, maximizing the power circulating in the arm cavities $[59,60]$.

The squeezing technique adopted by LIGO to improve the high-frequency sensitivity of the detectors is also implemented in the Virgo interferometer [61]. This system was fully operational also in Virgo for the first time during O3. To reduce the optical losses optimizing the squeezing performance, the photodiodes installed at the interferometer dark port to measure the gravitational-wave signals were replaced by high quantum efficiency ones [62].

Moreover, complementary activities have been carried out in parallel to the main upgrades, reducing the overall contribution of the various technical noises. In particular, the improvement of the control strategy for the suspended benches allows the reduction of the noise contribution below $30 \mathrm{~Hz}$, and the installation of baffles and diaphragms on the optical benches and inside the vacuum tanks reduces the impact of the scattered light on the sensitivity. Finally, sources of environmental noises have been identified and removed. All these upgrades increase the Virgo median BNS range by 1.73 (from 26 to $45 \mathrm{Mpc}$ ) with respect to the $\mathrm{O} 2$ run.

Figure 2 shows representative sensitivities of the three detectors during $\mathrm{O} 3 \mathrm{a}$, as measured by the amplitude spectral density of the calibrated strain output. Figure 3 shows the evolution of the detectors' sensitivity over time, as measured by the binary neutron star range. The up time of the detectors is kept as high as possible but is nevertheless limited by many factors, such as earthquakes, instrumental failures, and planned maintenance periods. The duty cycle for the three detectors is $76 \%$ (139.5 days) 


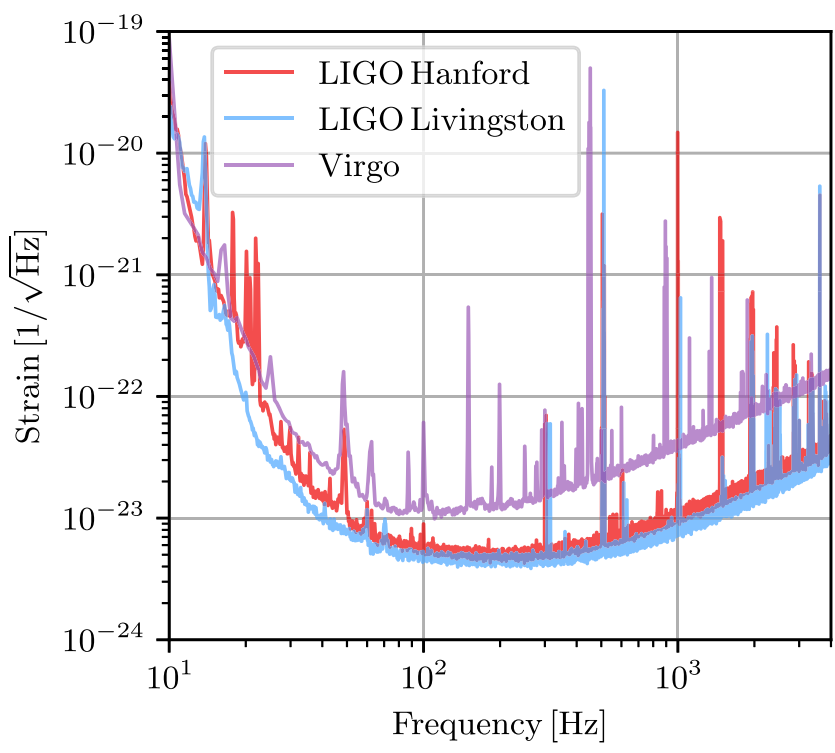

FIG. 2. Representative amplitude spectral density of the three detectors' strain sensitivity (LIGO Livingston 5 September 2019 20:53 UTC, LIGO Hanford 29 April 2019 11:47 UTC, and Virgo 10 April 2019 00:34 UTC). From these spectra, we compute BNS inspiral ranges of 109, 136, and $50 \mathrm{Mpc}$ for LIGO Hanford, LIGO Livingston, and Virgo, respectively.

for Virgo, 71\% (130.3 days) for LIGO Hanford, and 76\% (138.5 days) for LIGO Livingston. With these duty cycles, the full three-detector network is in observing mode for $44.5 \%$ of the time (81.4 days). Moreover, for $96.9 \%$ of the time (177.3 days), at least one detector is observing, and for $81.9 \%$ (149.9 days) at least two detectors are observing. For comparison, during the $\mathrm{O} 2$ run, the duty cycles are $62 \%$ for LIGO Hanford and $61 \%$ for LIGO Livingston, so that two detectors are in observing mode $46.4 \%$ of the time and at least one detector is in observing mode $75.6 \%$ of the time. The goal is, of course, to obtain the highest possible duty cycle. The current performance is limited by planned maintenance periods and the time needed to recover the control of the interferometers after large transients triggered, for example, by earthquakes. Work is ongoing at all detectors to improve the duty cycle.

\section{DATA}

Before analyzing LIGO and Virgo time-domain data for gravitational waves, we apply multiple data-conditioning steps to accurately calibrate the data into strain and mitigate periods of poor data quality [63]. Segments of data where each interferometer is operating in a nominal state, free from external intervention, are recorded [64]. Data from outside these time periods are not used in analyses unless additional investigations are completed to understand the state of the interferometer [6,33]. The data-conditioning process involves calibration of the data, both in near-real time and in higher latency; subtraction of noise from known instrumental sources; and identification of short-duration noise transients, which we refer to as glitches [65], that should be excluded from any searches for astrophysical candidates either by not considering the data containing the glitches or mitigating the glitches with methods such as gating.

For time periods containing gravitational-wave candidate events, additional investigation of the data quality is completed as a part of event validation to evaluate if instrumental artifacts could impact the detection and analysis of the candidate events [66]. These investigations sometimes lead to additional data-processing steps such as

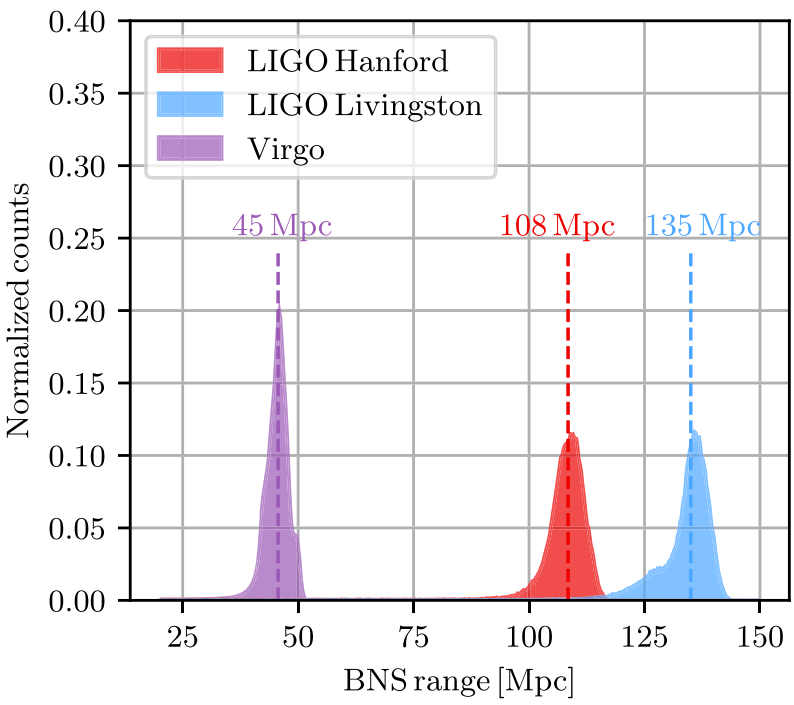

FIG. 3. The BNS range of the LIGO and Virgo detectors. Left: the evolution in time of the range over the entire duration of O3a. Each data point corresponds to the median value of the range over one-hour-long time segments. Right: distribution of the range and the median values for the entire duration of $\mathrm{O} 3 \mathrm{a}$. 
modeling and subtraction of glitches beyond what is completed to mitigate glitches before searching the data.

This section further outlines the procedures used to calibrate the data collected by LIGO and Virgo, characterize the data quality, validate any identified gravitationalwave candidates, and subtract glitches that may impact the analysis of candidates.

\section{A. Calibration and noise subtraction}

The optical power variations at the gravitational-wave readout ports of the LIGO and Virgo detectors are calibrated into a time series of dimensionless strain measured by the detectors before use by astrophysical analyses $[67,68]$. The calibration process requires data-conditioning filters whose response is complex valued, frequency dependent, and informed by detailed modeling of the feedback control system along with the interferometric, optomechanical response of the detectors [69]. Some control system model parameters vary slowly with time throughout operation of the interferometer. These parameters must be monitored, and, when possible, the filters are corrected in near-real time (low latency) [70]. Other parameters may change at discrete times and cause systematic error in the data stream that cannot be accounted for in low latency. Examples of such an error can arise from poorly compensated changes in electronics configurations, accidental application of incorrect control parameter values, model errors not yet known at the start of the observing period, and hardware problems such as failures of analog electronics within the control system. Most of these sources of error are subtle and can be assessed only once they are measured and quantified a posteriori.

All three detectors use photon calibrator (Pcal) systems [71-73] for absolute reference. These reference systems are used to develop each static detector model, measure parametric time dependence, and establish residual levels of systematic error in each strain data stream once constructed. Each of these measurements required to evaluate the systematic error is done by using the Pcal systems to drive forces on the end test masses via radiation pressure, creating displacement above other detector noise. Validating the strain data stream in low latency for all time, by establishing carefully quantified estimates of the systematic error with these excitations, competes with the desire for unhampered astrophysical sensitivity. As a compromise, systematic error is measured continuously only at a select few frequencies at the edges of the sensitive frequency band of the detector with monochromatic excitations during observation. The data stream is validated only at high-frequency resolution, and across the entire detection band, at roughly weekly cadence: The detectors are fully functional but are declared out of observation mode, and swept-sinusoid and colored-random-noise Pcal excitations are driven above the noise. These measurements can provide only approximate, point-estimate bounds on the data stream's error in low latency; they cannot reflect the error distribution for all time.

Once an observing period with a stable detector configuration is completed, estimates of the probability distribution of systematic error for all observation time are created [74]. These estimates leverage the power of hindsight, the collection of measurements mentioned above, and other measurements of individual components gathered while the detector is offline. During this error characterization process, if any identified systematic error is egregious and well quantified, where possible, the control system model and data-conditioning filters are modified to remove the error. The data stream is then regenerated offline from the optical power variations and control signals, and the systematic error estimate is updated accordingly [67].

Results in this paper are derived from either low-latency (C00) or offline, recalibrated (C01) strain data, depending on whether offline data were available and whether the results are sensitive to calibration error. Detection algorithms for gravitational-wave candidates, described in Sec. IV, are insensitive to typical levels of systematic error in calibration [75], so low-latency data may be used for additional offline analyses without concern. However, if available, offline data are preferred for improved accuracy and completeness. At the time the data were searched for candidate events, offline data were available only for a portion of O3a. The candidate events presented in this paper detected prior to 5 June 2019 are identified using LIGO offline data, whereas those from 5 June 2019 until 1 October 2019 are identified using LIGO low-latency data.

Once candidate events are found by detection algorithms using either LIGO data stream, all estimations of the candidates' astrophysical parameters use the C01 LIGO version of strain data using methods described in Sec. V. The C01 LIGO version of strain data was available for the entirety of $\mathrm{O} 3 \mathrm{a}$ at the time these additional analyses were completed. As such analyses are more sensitive to calibration error [76], it is advantageous to use the definitive characterization of error at the time of each event available with LIGO C01 data. The probability distributions of error for LIGO C01 strain data in O3a are characterized in Ref. [74]. Analysis of Virgo's collection of validation measurements during the run does not lead to a significant improvement to the low-latency strain data stream offline. As such, only lowlatency strain and its bounds on systematic error from pointestimate measurements are used for all detection and astrophysical parameter estimation results presented in this paper. The bounds of systematic error of Virgo strain in O3a are reported in Ref. [77].

Numerous noise sources that limit detector sensitivity are measured and linearly subtracted from the data using witness auxiliary sensors that measure the source of the noise $[41,78,79]$. In O3a, the sinusoidal excitations used for calibration and noise from the harmonics of the power mains are subtracted from LIGO data as a part of the 
calibration procedure [79]. This subtraction is completed for both online and offline versions of the calibration. For time periods around a subset of identified candidate events, additional noise contributions due to nonstationary couplings of the power mains are subtracted [80].

The Virgo online strain data production also performs broadband noise subtraction during O3a [77]. The subtracted noises include frequency noise of the input laser, noise introduced controlling the displacement of the beam splitter, and amplitude noise of the $56 \mathrm{MHz}$ modulation frequency. An additional offline strain dataset is produced for 14 September 2019 through 1 October 2019 using the same calibration as the online data but with improved noise subtraction, resulting in a BNS range increase of up to $3 \mathrm{Mpc}$ [81], and is used in source parameter estimation of candidate events that occur during this time period.

\section{B. Data quality}

During O3a, the data quality is closely monitored using summarized information from the detectors and their subsystems [82,83]. Deeper studies are conducted to identify the causes of data quality issues, which enable instrumental mitigation of the sources during the run. For example, at Livingston, glitches from a mechanical camera shutter and beats of varying radio-frequency signals are identified and eliminated. At Hanford and Livingston, strong frequency peaks that wander in time are tracked down to the amplitude stabilizer for the laser used to provide squeezed light. At Hanford, broad features in the spectrum at $48 \mathrm{~Hz}$ and multiples are tracked to scattered light from vacuum chamber doors and mitigated with absorptive black glass. These studies are a part of ongoing efforts to improve the data quality and the up time of the detectors $[40,82]$.

For analyses of gravitational-wave transients, the most common data quality issue is the presence of glitches. The rate of glitches with signal-to-noise ratio $(\mathrm{SNR})>6.5$ in the LIGO and Virgo detectors in O3a is shown in Fig. 4. In all three detectors, the glitch rate is dominated by glitches with peak frequencies below $100 \mathrm{~Hz}$. This rate is higher than in previous observing runs [66] for both LIGO detectors and is especially problematic at LIGO Livingston, where the rate of glitches is 0.8 per minute in $\mathrm{O} 3 \mathrm{a}$, compared to 0.2 per minute in $\mathrm{O} 2$. The Virgo glitch rate decreases significantly between $\mathrm{O} 2$ and $\mathrm{O} 3 \mathrm{a}$, thanks to the work done during the $\mathrm{O} 2-\mathrm{O} 3$ a shutdown to improve the accuracy of Virgo's operating point control and to identify, fix, or mitigate several sources of noise. The increased rate of glitches in the LIGO detectors limits the overall sensitivity of searches for gravitational waves in O3a and creates challenges for analysis of candidate events.

The most problematic source of glitches in $\mathrm{O} 3 \mathrm{a}$ is caused by laser light scattered out of the main laser beam, which is reflected off walls of the vacuum systems and other equipment back into the main beam [52,86-88].
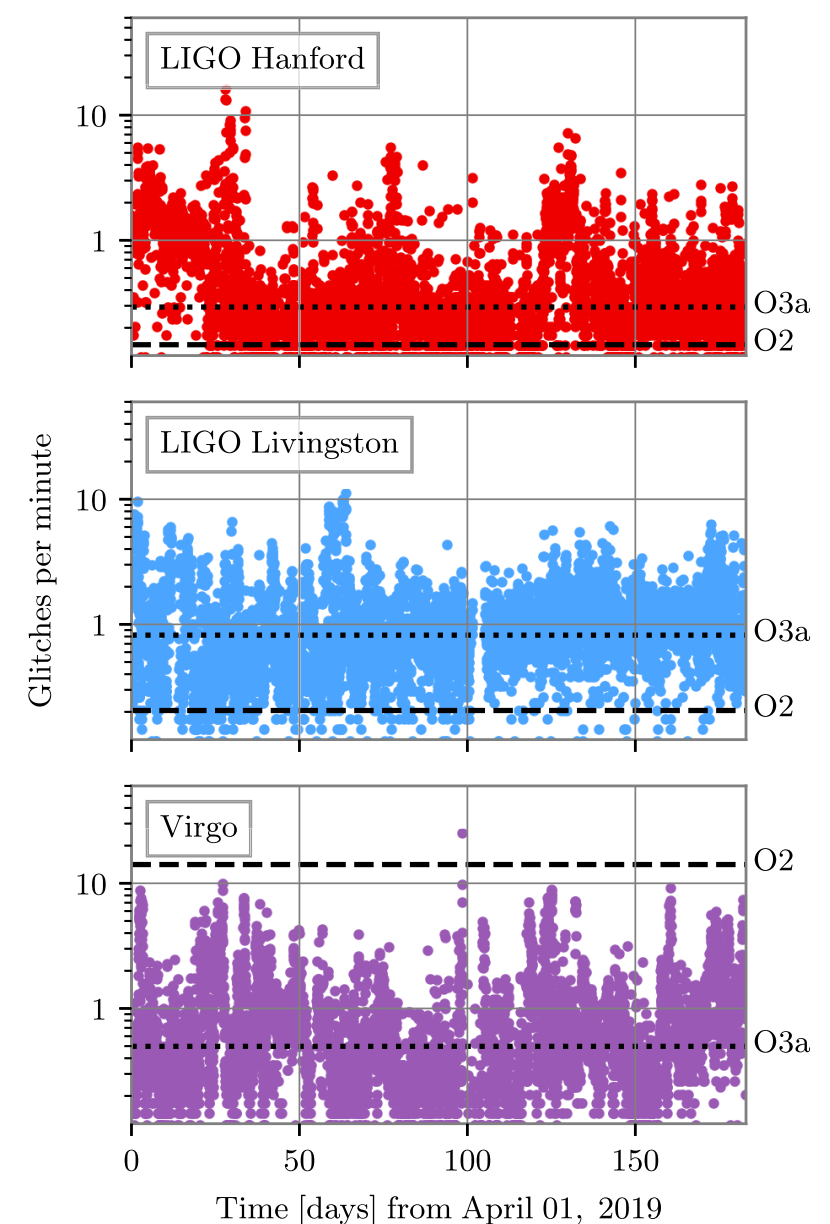

FIG. 4. The rate of single interferometer glitches with SNR $>6.5$ and frequency between 10 and $2048 \mathrm{~Hz}$ identified by Omicron $[84,85]$ in each detector during O3a. Each point represents the average rate over a 2048 s interval. Dotted black lines show the median glitch rate for each detector in $\mathrm{O} 2$ and $\mathrm{O} 3 \mathrm{a}$.

Scattered light noise is correlated with periods of high seismic activity. For this reason, daily cycles of scattered light glitches are present throughout $\mathrm{O} 3 \mathrm{a}$, especially at LIGO Livingston, tied to ground motion driven by human activity. This noise is often visible as arch-shaped features in time frequency [86,89], as shown in Fig. 5, and is present at or near the time of many of the candidate events in this catalog. A significant portion of the increase in glitch rate between $\mathrm{O} 2$ and $\mathrm{O} 3 \mathrm{a}$ at the LIGO detectors can be accounted for by the increased rate of scattered light glitches [88]. A potential major source of this noise for O3a is light scattered from the gold-coated electrostatic drives mounted to the fused silica reaction masses that are suspended directly behind the LIGO test masses to provide a stable platform for low-noise actuation [88]. Changes are implemented during $\mathrm{O} 3 \mathrm{~b}$ that reduce scattered light noise entering through this path [88].

Broadband short-duration glitches also occur often in all detectors during O3a. A subclass of those, blip 


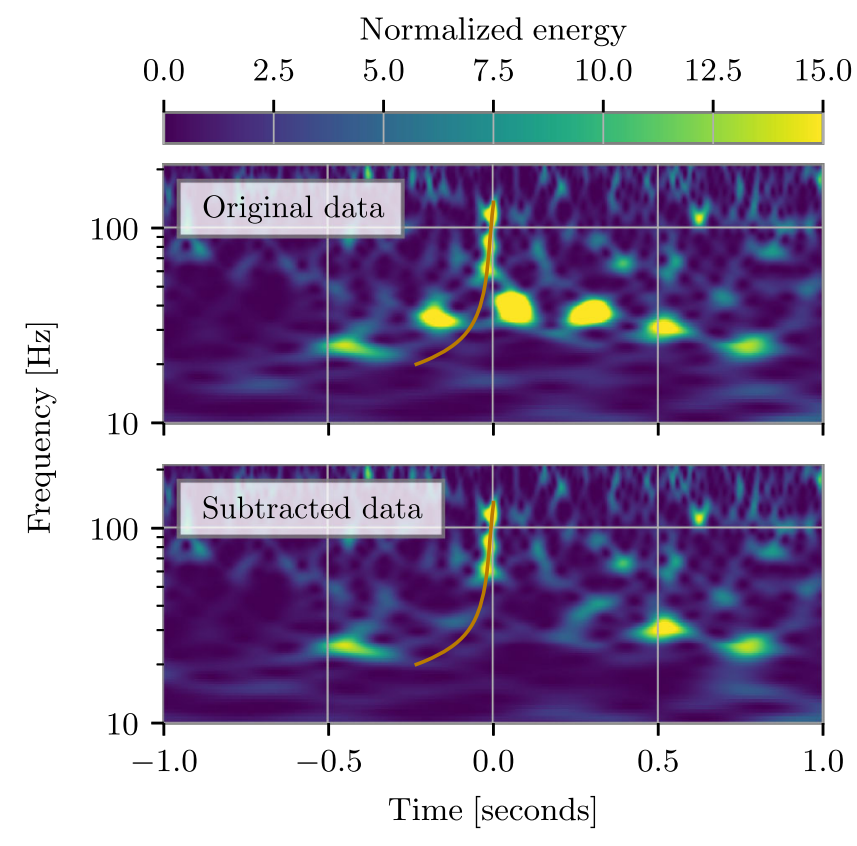

FIG. 5. Top: time-frequency representation of the data surrounding event GW190701_203306 at LIGO Livingston, showing the presence of scattered light glitches (modulated arches). Bottom: the same data after glitch identification and subtraction as described in Sec. III D. In both plots, the time-frequency track of the matched-filter template used to identify GW190701_ 203306 is overlaid in orange. Investigations identify seven candidate events in coincidence with similar scattering glitches and require mitigation before further analysis. Despite the clear overlap of the signal with the glitch, the excess power from the glitch is successfully modeled and subtracted.

glitches [90], is one of the most problematic sources of transient noise in previous observing runs and is still present in $\mathrm{O} 3 \mathrm{a}$ at a rate of 1.4 per hour. These glitches are one of the limiting sources of noise for searches for gravitational waves from high-mass compact binaries [91], and no sources or witnesses for the majority of these glitches have been identified. In O3a, there was also an additional population of short-duration glitches with SNR > 100. These loud glitches occur in both LIGO detectors, with unknown origin. Additional description of these glitches, along with details of potential sources that have been ruled out, can be found in Ref. [82].

Many glitches in LIGO and Virgo data have wellunderstood sources and couplings, making it possible to identify short time periods where excess power from environmental or technical sources is present in the strain data. Flagging these time periods as containing poor data quality, either by removing the data from the search or decreasing the significance of any candidate events identified, is shown to improve the overall sensitivity of searches for gravitational waves from compact binaries $[82,92,93]$. While a select number of LIGO and Virgo data quality issues are flagged in low latency, such as hardware
TABLE I. Percent of single-detector time removed by each category of veto for each detector. Category 1 vetoes are applied in all analyses described in Sec. IV. CBC category 2 vetoes are applied only by the РусвC search. Burst categories 2 and 3 are applied only by the cWB search.

\begin{tabular}{lcccc}
\hline \hline Detector & $\begin{array}{c}\text { Category } \\
1\end{array}$ & $\begin{array}{c}\text { CBC } \\
\text { category }\end{array}$ & $\begin{array}{c}\text { Burst } \\
\text { category }\end{array}$ & $\begin{array}{c}\text { Burst } \\
\text { category } 3\end{array}$ \\
\hline $\begin{array}{l}\text { LIGO } \\
\text { Hanford }\end{array}$ & $0.27 \%$ & $0.37 \%$ & $0.83 \%$ & $0.19 \%$ \\
$\begin{array}{c}\text { LIGO } \\
\text { Livingston }\end{array}$ & $0.08 \%$ & $0.10 \%$ & $0.64 \%$ & $0.15 \%$ \\
Virgo & $0.15 \%$ & $\ldots$ & $\ldots$ & $\ldots$ \\
\hline \hline
\end{tabular}

injections and digital signal overflows, the majority of data quality flags are available only for offline searches.

Before performing searches for gravitational-wave signals, periods of poor data quality are flagged at various levels, called categories $[63,66,82]$. In O3a, data from an observatory not operating in a nominal state are flagged (category 1) and not used in any search. Additional periods likely to contain excess power in each LIGO detector are flagged based on detailed follow-up of identified sources of noise (category 2), statistical correlation between witness sensors (category 3), and machine-learning-based predictions (iDQ) $[93,94]$. No additional data quality products for Virgo are used beyond category 1 . The specific set of data quality products used in O3a is search specific, as described in Sec. IV. Category 2 flags are tuned separately for searches for both gravitational waves from $\mathrm{CBC}$ and minimally modeled (burst) sources. Category 3 flags are tuned only for burst searches. The amount of time removed by each category of veto in O3a is shown in Table I.

\section{Event validation}

Event validation procedures similar to those used for previous gravitational-wave candidate events $[8,66,82]$ are used for all candidate events in this catalog to evaluate if instrumental artifacts could impact analysis. Within tens of minutes of low-latency candidate event identification, timefrequency visualizations and monitors of the gravitationalwave strain data [95-100] are used to identify any data quality issues present. On the same timescale, data from hundreds of auxiliary sensors monitoring the detectors and their environments are used to identify potential auxiliary witnesses to instrumental artifacts [94,101,102]. Tools that rely upon deeper information about glitches and data nonstationarity gathered offline, such as long-term monitors of the instruments and their subsystems [83,103,104] and identification of likely sources of glitches by correlation with auxiliary sensors $[85,105,106]$, are also used to vet candidate events in this catalog. These procedures do not identify evidence of instrumental origin for any of the candidate events in this catalog but do identify a number of 
data quality issues that could potentially impact analyses of these candidate events.

Candidate events with data quality issues identified by these event validation procedures require further mitigation before analysis. In cases when glitches occur in time coincidence with a candidate event (but cannot account for the candidate event itself), additional data-processing steps are completed to mitigate the effect of those glitches on estimation of the candidate event parameters. If possible, the identified glitches are subtracted using the methods described in Sec. III D. In cases when sufficient subtraction is not possible, customized configurations of parameter estimation analyses are used to exclude the time period or frequency bandwidth impacted by glitches. An example of a glitch coincident with a signal that requires glitch subtraction is shown in Fig. 5. While the presence of excess power from transient noise does not prevent confident identification of this event, glitch subtraction is required before the source properties of the event can be evaluated. Although only data recorded from detectors in observing mode are used to identify candidate events in this catalog, some candidate events occur at times when one detector in the network is operating but not in observing mode. If it is concluded that data from the additional detector would substantially impact the scientific conclusions reached from analyzing the candidate, the additional data are investigated. For those cases, the data quality and calibration for the nonobserving detector are evaluated to determine whether the data could be used in the estimation of candidate event source properties. Such data are used for one candidate event, GW190814 [33]. The full list of candidate events requiring specific mitigation steps, due to either the presence of glitches or the state of a detector, is found in Sec. VII. The total number of candidate events requiring mitigation is consistent with the number expected based on the glitch rate in each detector.

\section{Glitch subtraction}

Data containing gravitational-wave candidate events and glitches in the same time-frequency volume are preprocessed through a glitch-subtraction procedure prior to being analyzed by the parameter estimation pipelines. The glitchsubtraction procedure evolved from the BayesWave (BW) algorithm [107-109] used for glitch subtraction in the Livingston detector at the time of the GW170817 binary neutron star merger [12,110], where the non-Gaussian, incoherent, noise is modeled as a linear combination of wavelets which is subtracted from the data. The number of wavelets used in the fit is determined using a transdimensional Markov chain Monte Carlo (MCMC) algorithm that balances using fewer wavelets against the quality of the fit [107].

To prevent the glitch-subtraction procedure from corrupting the signal candidate event, the time segment and bandwidth of the wavelet-based analysis are chosen, when possible, to exclude from subtraction the strongest part of the signal. For cases where the signal and glitch overlap in time-frequency space, a more robust application of the glitch-subtraction algorithm is used which simultaneously fits for the signal and the glitch. In the signalplus-glitch application, signal wavelets are included in the model if they are coherent over the detector network (marginalizing over sky location, etc.), while the glitch wavelets are independent in each detector [108]. Only the glitch model wavelets are then used in the subtraction. The signal-plus-glitch procedure is tested by injecting simulated coherent BBH signals onto known singledetector glitches from $\mathrm{O} 2$ and verifying that the signals are unaffected in the process.

The glitch-subtraction procedure is used only as a preprocessing step for the parameter estimation analysis (described in Sec. V) and is not part of the analyses that determine the presence, or significance, of gravitationalwave candidate events. As shown in Fig. 5, the glitchsubtraction methods described here are able to successfully remove excess power caused by glitches present near the time of candidate events.

\section{CANDIDATE IDENTIFICATION}

Candidate identification happens on two timescales. First, five low-latency gravitational-wave pipelines [111-115] process the data immediately after acquisition with the goal of generating public detection alerts to the broader astronomical community within minutes [116]. Second, an offline reanalysis of gravitational-wave data is conducted to produce the curated candidate event list here. The offline analysis may benefit from updated data calibration, data quality vetoes, the ability to estimate event significance from the full data, and further algorithmic development that takes place over the course of an observing run. Although the candidate events presented here are derived from offline analysis, we provide a comparison with the public alerts in Sec. VI. Candidates are identified using two methods. The first method searches for minimally modeled sources. The second method searches for signals from a bank of template waveforms [117] modeled after the expected gravitational-wave emission from coalescing compact binaries in general relativity. In Sec. VI, we present results from one search for minimally modeled transient sources, coherent WaveBurst (cWB) [112,118-121], and two searches for modeled sources, GstLAL [111,122,123] and РуСвC [29,124-127]. cWB, GstLAL, and РyСBC are also three of the five low-latency pipelines in $\mathrm{O} 3$. The two remaining low-latency pipelines, MBTAOnline [114,128] and SPIIR [115], were not configured for offline reanalysis at the time of this publication and are, therefore, not included in GWTC-2. Below, we summarize the methods used by each of cWB, GstLAL, and РyСBC to identify candidate events. 


\section{A. Coherent WaveBurst search for minimally modeled transient sources}

$\mathrm{cWB}$ is a search pipeline for detection and reconstruction of transient GW signals that operates without a specific waveform model [129] and was used in previous searches by the LVC $[1,8,130]$. cWB identifies coincident signal power in multiple detectors, searching for transient signals with durations up to a few seconds in the detector bandwidth. The analysis is performed on the timefrequency data obtained with the Wilson-DaubechiesMeyer wavelet transform $[118,131]$ and normalized by the amplitude spectral density of the detector noise. cWB selects the time-frequency data samples above fluctuations of the detector noise and groups them into clusters. For clusters correlated in multiple detectors, cWB reconstructs the source sky location and signal waveforms with the constrained maximum likelihood method [112]. The signal SNR is estimated from the signal waveforms reconstructed by cWB, and the network SNR is calculated combining the SNRs of individual detectors.

The cWB detection statistic is based on the coherent energy $E_{c}$ obtained by cross-correlating the normalized signal waveforms reconstructed in different detectors. It is normalized by a chi-squared statistic $\chi^{2}=E_{n} / N_{\mathrm{df}}$, where $E_{n}$ is the residual noise energy estimated after the reconstructed waveforms are subtracted from the data and $N_{\mathrm{df}}$ is the number of independent wavelet amplitudes describing the event. The cWB detection statistic is $\eta_{c} \propto\left[E_{c} / \max \left(\chi^{2}, 1\right)\right]^{1 / 2}$, where the $\chi^{2}$ correction is applied to reduce the contribution of non-Gaussian noise. To improve the robustness of the algorithm against glitches, cWB uses signal-independent vetoes, which reduce the FAR of the pipeline; this includes category 2 burst data quality flags in the processing step and hierarchical vetoes in the postproduction phase [105,132]. Other vetoes applied to candidate events are on the network correlation coefficient $c_{c}=E_{c} /\left(E_{c}+E_{n}\right)$ and the $\chi^{2}$. To further reduce the background, the cWB analysis employs additional signal-dependent vetoes based on the properties of the time-frequency evolution of compact binary signals: (a) The frequency of the signal is increasing in time [133], and (b) the central frequency of the signal $f_{c}$ is inversely proportional to the total mass of the system [134]. cWB searches are performed with two pipeline configurations targeting detection of high-mass $\left(f_{c}<80 \mathrm{~Hz}\right)$ and lowmass $\left(f_{c}>80 \mathrm{~Hz}\right)$ BBH systems. They use different signal-dependent vetoes defined a priori to alleviate the large variability of nonstationary noise in the detectors' bandwidth.

We estimate the significance of candidate events by systematically time shifting the data of one detector with respect to the other in each detector pair, with a time lag so large that actual astrophysical events are excluded, and repeating this for a large number of different time lags over a total time $T_{\text {bkg }}$. We count the number of events $N$ due to instrumental noise that have a ranking statistic value, such as the SNR that is at least as large as that of the candidate event, and we compute the FAR as the number of background events divided by the total background time [135]. The detection significance of a candidate event identified by either configuration in a single-frequency range is determined by its FAR measured by the corresponding cWB configuration. Whenever the low-mass and high-mass configurations overlap, the trials factor of 2 is included to determine the final FAR [34]. In the end, each configuration reports the selected candidate events and their FAR.

The sensitivity of the cWB search pipeline approaches that of matched-filter methods for coalescing stellar mass BBHs with high chirp masses, where most of the signal energy is concentrated in just a few wavelets of the cWB representation [136]. It is less competitive for low-chirpmass events, where the signal power is spread over large time-frequency areas. cWB can also detect sources that are not well represented in current template banks (e.g., eccentric systems or high-mass ratio precessing systems) [137]. Tests with cWB show that the detection efficiency for a given FAR threshold is slightly smaller with the inclusion of Virgo. Therefore, also to reduce computing time, all cWB detection candidates and waveform consistency tests reported in this catalog use the HanfordLivingston network only.

\section{B. GstLAL and PyCBC searches for modeled sources}

Both the GstLAL pipeline $[111,122,123]$ and the PyCBC [138] pipeline [29,124-127] implement independently designed matched-filter analyses. Both are used in previous LVC searches for gravitational waves [1,4-8,39].

The matched-filter method relies on a model of the signal, dependent on the source physical parameters. Most important for the phase evolution of the source (and, therefore, the matched filter) are the intrinsic parameters: two individual component masses $m_{1}$ and $m_{2}$ and two dimensionless spin vectors $\vec{\chi}_{\{1,2\}}$, where the dimensionless spin is related to each component's spin angular momentum $\vec{S}$ by $\vec{\chi}_{i}=c \vec{S}_{i} /\left(G m_{i}^{2}\right)$.

We also make use of combinations of these intrinsic parameters that are typically well constrained by gravitational-wave measurements; the binary chirp mass [139]

$$
\mathcal{M}=\frac{\left(m_{1} m_{2}\right)^{3 / 5}}{\left(m_{1}+m_{2}\right)^{1 / 5}}
$$

determines to lowest order the phase evolution during the inspiral and is typically better constrained than the component masses. At higher orders, the mass ratio $q=m_{2} / m_{1}$ (where $m_{2} \leq m_{1}$ ) and effective inspiral spin $\chi_{\text {eff }}$ affect the binary phase evolution. The effective inspiral spin is defined as [140] 


$$
\chi_{\mathrm{eff}}=\frac{\left(m_{1} \vec{\chi}_{1}+m_{2} \vec{\chi}_{2}\right) \cdot \hat{L}_{N}}{M},
$$

where $M=m_{1}+m_{2}$ is the total mass and $\hat{L}_{N}$ is the unit vector along the Newtonian orbital angular momentum. The spin tilt angle for each component object $\theta_{\mathrm{LS}_{i}}=$ $\cos ^{-1}\left(\vec{\chi}_{i} \cdot \hat{L}_{N} /\left|\vec{\chi}_{i}\right|\right)$ quantifies the angle between the orbital angular momentum vector and its spin vector. Since the spin and angular momentum vectors vary if the system precesses, by convention we use the spin parameters at a reference frequency of $20 \mathrm{~Hz}$, with the exception of GW190521, where we use $11 \mathrm{~Hz}$ for consistency with previous publications $[34,35]$. Additional intrinsic parameters are needed to describe eccentricity, which we assume to be zero in our modeled analyses. The timescale for circularization of isolated binaries with nonzero eccentricity at birth is sufficiently short that sources are expected to have negligible eccentricity when they enter the sensitive bands of the LIGO and Virgo detectors [141]. However, dynamically formed binaries may have residual eccentricity as the signal enters the sensitive band of the detector. These systems are the target of unmodeled searches of previous observing runs but with no candidate events reported [137].

Seven extrinsic parameters provide the orientation and position of the source in relation to Earth: the luminosity distance $D_{L}$, two-dimensional sky position (right ascension $\alpha$ and declination $\delta$ ), inclination between total angular momentum and line of sight $\theta_{J N}$, time of merger $t_{c}$, a reference phase $\phi$, and polarization angle $\psi$.

In general, as the signal travels from the source to the detector, its frequency is redshifted by a factor of $(1+z)$. For a system involving only black holes, the observed signal is identical to that from a source in the rest frame of the detector with total mass $M^{\text {det }}=(1+z) M[142,143]$. For convenience, the templates used by the modeled searches are defined in the rest frame of the detectors which subsumes the factor $(1+z)$ into the definition of masses.

For this work, the GstLAL analysis uses a template bank with component masses between $1 M_{\odot}$ and $400 M_{\odot}$ with total masses $M^{\text {det }}$ between $2 M_{\odot}$ and $758 M_{\odot}$ and spins that are aligned or antialigned with the binary's orbital angular momentum, such that only the spin components $\chi_{i, z}=\vec{\chi}_{i} \cdot \hat{L}_{N}$ are nonzero. The bank is constructed in five regions via a stochastic placement algorithm [144,145] satisfying different minimal match (MM) [117] criteria with waveforms starting at $f_{\min }$ as described in Table II. Template placement is augmented to improve the collection of background statistics in the last region shown in Table II by a grid of templates distributed uniformly in the logarithm of component mass to improve detection efficiency for systems with primary mass $m_{1}^{\text {det }}$ above $50 M_{\odot}[8,146]$. The TaylorF2 waveform approximant [139,147-156] is used for templates with $\mathcal{M}^{\text {det }}<1.73 M_{\odot}$ and the
TABLE II. GstLAL template bank parameters. Low spin denotes the range -0.05 to 0.05 , and high spin denotes the range -0.999 to 0.999 .

\begin{tabular}{cccccccc}
\hline \hline MM & $m_{1}$ & $m_{2}$ & $M$ & $q$ & $\chi_{1, z}$ & $\chi_{2, z}$ & $f_{\min }(\mathrm{Hz})$ \\
\hline 0.99 & 1,3 & 1,3 & $<6$ & $0.33,1$ & Low & Low & 15 \\
0.97 & 3,150 & 1,3 & $<153$ & $0.02,1$ & High & Low & 15 \\
0.99 & 3,91 & 3,50 & $<100$ & $0.1,1$ & High & High & 15 \\
0.97 & 30,392 & 3,36 & $<400$ & $0.02,0.1$ & High & High & 15 \\
0.99 & 50,400 & 9,400 & $>100$ & $0.1,1$ & High & High & 10 \\
\hline \hline
\end{tabular}

SEOBNRv4_ROM waveform approximant [157] for templates with $\mathcal{M} \geq 1.73 M_{\odot}$.

The $\mathrm{PyCBC}$ analysis uses a template bank covering the same parameter space as for GWTC-1 [8] shown in Figs. 3 and 7 of Ref. [158]. Unlike the previous work, the template bank here is created using a hybrid geometric-random method described in Refs. $[159,160]$. This new method provides a more efficient template bank-in terms of covering the full parameter space with fewer template waveforms-than the stochastic method $[144,145]$. This bank is broadly similar to the parameter space covered in the GstLAL search described above with a key difference being that only templates longer than $0.15 \mathrm{~s}$ are kept. Details about this cut and its effect on the explored mass and spin range can be found in Ref. [158].

Both GstLAL and PyCBC scan data from each gravitational-wave detector against the above-described banks of template waveforms to produce SNR time series [29]. The SNR time series are maximized over short time windows to produce a set of triggers for each template and each detector. Triggers that pass an SNR threshold of 4 in one detector form the basis of candidate events according to the procedures for each pipeline described below. РуСВС removes the time period during category 2 veto flags from the final results, while GstLAL uses only iDQ for singledetector triggers and no data quality products for coincident triggers.

GstLAL defines a candidate event as consisting of triggers from one or more gravitational-wave detectors ranked by the SNRs of the triggers, signal-consistency tests, time delays between each detector, phase differences between detectors, the (possibly zero) time-averaged volumetric sensitivity of each detector, and the signal population model. These parameters are used as variables in the likelihood-ratio ranking statistic $\mathcal{L}[111,122,123,161]$, which is a monotonic function of the inverse false alarm probability [162].

There are two differences between the ranking statistic used here and in $\mathrm{O} 2$ [8]. First, we implement a template likelihood $p(T \mid$ signal, SNR) [163], which is the probability that a trigger is recovered by a template $T$, given the trigger SNR and that the signal belongs to some population. Previous versions of GstLAL approximate $p(T \mid$ signal, SNR) by a constant in $\mathcal{L}[111,122,161]$, implying all templates are equally likely to recover a signal. Now, the 
template likelihood is informed by the template bank (to account for the fact that templates are not uniformly distributed in parameter space [164]) and a signalpopulation model, which for this search considers $\vec{\theta}=$ $\left\{m_{1}^{\text {det }}, m_{2}^{\text {det }}, \chi_{1, z}, \chi_{2, z}\right\}$ and is given by $p(\vec{\theta} \mid$ signal $) d \vec{\theta} \propto$ $1 /\left(4 m_{1}^{\text {det }} m_{2}^{\text {det }}\right) d \vec{\theta}$. The distribution $p(\vec{\theta} \mid$ signal $)$ is deliberately broad to minimize the number of missed signals. Second, single-detector candidate events are ranked using both an empirically determined penalty and information from iDQ [94] as described previously. The penalty from $\mathrm{iDQ}$ is added to the denominator of $\mathcal{L}$. Candidates from the GstLAL search have their likelihood ratios and significance estimated using the entire approximately 6-month dataset.

РуСВC identifies candidate events by requiring triggers in both LIGO Hanford and LIGO Livingston with a time delay smaller than the light travel time between observatories. These candidate events are then ranked using a set of signal-based vetoes, data quality information, and by comparing the properties of the event against those expected from astrophysical signals [127]. A FAR is then computed for each of these candidate events by estimating the background noise distribution using timeshifted analyses similarly to cWB $[29,126,165]$, triggers from LIGO Livingston being time shifted relative to LIGO Hanford by multiples of $0.1 \mathrm{~s}$. Virgo data are not searched with the РуСвС pipeline due to three-detector searches not being completely integrated in the version of code used.

A focused search for BBH coalescences [27] is also used here, denoted later as $\mathrm{PyCBC} \mathrm{BBH}$. This search is motivated by the fact that all signals observed in $\mathrm{O} 1$ and $\mathrm{O} 2$, with the single exception of the binary neutron star merger GW170817, are consistent with BBH coalescences with mass ratio close to 1 and effective inspiral spins close to 0 . The full parameter space search used for GWTC-1 [8], in contrast, is tuned to observe signals anywhere in the possible space of signal parameters, which might include signals that do not have very high matches with search templates. The PyCBC BBH search uses a recently developed detection statistic $[27,166]$ which includes a number of tuning choices to reject triggers that do not match the filter waveforms well and also includes a template weighting implementing a prior that signals detectable in any given range of SNR are uniformly distributed in chirp mass. This search enables PyCBC to identify more BBHs in the O1 and $\mathrm{O} 2$ datasets than reported in the GWTC-1 paper $[8,27]$. This search includes some of the BBHs first reported in Refs. [23,25] by independently developed searches $[24,167]$. We use the focused BBH search in this work to better extract $\mathrm{BBH}$ coalescences from the data: This search considers only a reduced set of filter templates defined prior to the analysis of $\mathrm{O} 3$ data, namely, systems with mass ratio $q>1 / 3$ and with both component masses (in detector frame) larger than $5 M_{\odot}$.

\section{Estimation of modeled search sensitivity}

In order to estimate the sensitivity of the GstLAL and PyCBC searches, we conduct a campaign of simulated signals injected into the $\mathrm{O} 3 \mathrm{a}$ gravitational-wave data and analyzed by both matched-filter pipelines. The simulated population, intended to cover (or overcover) the detected population of stellar-mass BBHs [11,37], contains component masses $m_{1}$ and $m_{2}$ between $2 M_{\odot}$ and $100 M_{\odot}$ and extends out to a maximum redshift of 2.3. In order to reduce statistical uncertainties, the mass, spin, and redshift distributions should be sufficiently similar to population models for which we intend to estimate merger rates: See Ref. [37] (Appendix A) for further discussion of selection functions in population inference. For the simulations, we choose $p\left(m_{1}\right) \propto m_{1}^{-2.35}, p\left(m_{2} \mid m_{1}\right) \propto m_{2}^{2}$ (for $\left.m_{2}<m_{1}\right)$, and $\chi_{i, z}$ values distributed uniformly between -0.998 and 0.998 . The cosmological distribution of sources simulates a merger rate in the comoving frame that evolves as $R(z)=R(0)(1+z)^{2}$; thus, the source redshift distribution follows $p(z) \propto(1+z) d V_{c} / d z$, where $V_{c}$ is the comoving volume (see, e.g., Ref. [168] for further discussion of cosmological effects). The simulation set is generated in two stages: First, points are chosen according to this distribution out to $z=2.3$; then, these $7.70 \times 10^{7}$ samples are reduced to a set of potentially detectable signals by imposing that the expected LIGO HanfordLIGO Livingston network SNR, calculated using representative noise power spectral density (PSD), be above a threshold of 6. The approximately 157000 signals remaining after this cut are assigned merger times ranging uniformly over the duration of $\mathrm{O} 3$ for analysis by the searches. The SEOBNRv4_opt aligned-spin waveform model [157] is used for the simulated signals.

The expected number of signals from such a population detected by a given analysis may be written as

$$
\hat{N}=\mathcal{V} R(0),
$$

where $R(0)$ is the rate of signals per unit volume and unit observing time at present and $\mathcal{V}$ is the effective surveyed hypervolume, a measure of analysis sensitivity for the injected population [169]. Since each analysis recovers over $10^{4}$ injections, statistical counting uncertainties in the hypervolumes $\mathcal{V}$ are at the subpercent level. Our estimates of sensitivity are, though, affected by possible systematic uncertainties in calibration of the strain data. Strain calibration affects the detectability of simulated signals via the magnitude of the response function, which is affected by uncertainties of at most a few percent over the frequency range where the SNR of binary merger signals is accumulated; see Ref. [74] for details. The hypervolume $\mathcal{V}$ surveyed by each analysis, at a detection FAR threshold of 2.0 per year, is $0.456 \mathrm{Gpc}^{3} \mathrm{yr}$ for GstLAL, $0.296 \mathrm{Gpc}^{3} \mathrm{yr}$ for $\mathrm{PyCBC}$, and $0.386 \mathrm{Gpc}^{3} \mathrm{yr}$ for $\mathrm{PyCBC}$ $\mathrm{BBH}$. For a combination of all matched-filter analyses 
presented in this catalog, with any injection found in one or more analysis below the FAR threshold considered as detected, we find a surveyed hypervolume $0.567 \mathrm{Gpc}^{3} \mathrm{yr}$.

\section{Estimation of signal probability}

For each candidate event, the probability of origin from an astrophysical source $p_{\text {astro }}$ and corresponding probability of terrestrial noise origin $p_{\text {terr }}=1-p_{\text {astro }}$ may be estimated using the outputs of search pipelines. We obtain these probabilities for the candidate events in this catalog consistent with a $\mathrm{BBH}$, via the Poisson mixture model formalism [170] used in O1 [39,168,171]. Only BBH candidate events are considered because, other than GW190425 [172] whose component masses are consistent with those of NSs, all significant detections can be classified as BBHs [173]. A low-significance candidate NSBH event, GW190426_152155, is reported in low latency [174]; however, its astrophysical probability is strongly dependent on prior assumptions of the rate of such signals. We, therefore, do not estimate its $p_{\text {astro }}$ here.

We start by collecting the ranking statistics $\vec{x}=$ $\left\{x_{1}, x_{2}, \ldots, x_{N}\right\}$ of all candidate events more significant than a predefined threshold: For the GstLAL pipeline events are thresholded on FAR, while for $\mathrm{PyCBC}$ a ranking statistic threshold is applied. The threshold for GstLAL is chosen to ensure that the total number of background events considered exceeds the number of signals by a large (approximately 100) factor, which enables an accurate estimate of the total rate of background events above threshold. On the other hand, $\mathrm{PyCBC}$ estimates the background rate from timeshifted analyses, as outlined above in Sec. IV B; thus, the requirement to include a large number of background events is relaxed. The statistic threshold is then set low enough to include (at least) all events with $p_{\text {astro }} \gtrsim 0.1$.

Additionally, for both GstLAL and PyCBC searches, a threshold of $4.35 M_{\odot}$ is applied on the chirp mass of the templates, corresponding to a $5 M_{\odot}+5 M_{\odot}$ binary, ensuring that the selected candidate events have template masses consistent with those of putative BBHs. Using the distribution of ranking statistics $x$ under the foreground model, $f(x)=p(x \mid$ signal $)$, and the distribution under the background model, $b(x)=p(x \mid$ noise $)$, estimated by each matched-filter pipeline, we assign a Bayes factor $k(x)=$ $f(x) / b(x)$ to each event. Assuming that foreground and background triggers are drawn from independent Poisson processes, one can then calculate the posterior over the Poisson expected counts for each process, $\Lambda_{1}$ and $\Lambda_{0}$.

For the PyCBC searches presented here, we proceed as for $\mathrm{O} 1$ and $\mathrm{O} 2$ [8] and estimate foreground and background event densities empirically for all putative BBH candidate events with ranking statistic above a given threshold. The PyCBC full parameter space and $\mathrm{BBH}$ focused searches differ in how their ranking statistics are calculated: For the full search [127], a threshold of 7.9 is applied to the ranking statistic, while for the BBH search [27,166], a threshold value of 9 is applied. We empirically measure the rate of noise events satisfying these cuts via time-shifted analyses and infer the posterior over the rate of signals; finally, we marginalize over the signal rate to obtain probabilities of astrophysical and terrestrial origin for each event [175]. Since the PyCBC BBH search is more sensitive to realistic $\mathrm{BBH}$ signal populations, implying a more accurate estimate of the relative densities of signal and noise events within its targeted mass region, we consider the $p_{\text {astro }}$ values from the $\mathrm{BBH}$ analysis to be more accurate for events recovered by both searches.

For the GstLAL analysis, we estimate the astrophysical and terrestrial probabilities $p_{\text {astro }}(x \mid \vec{x})$ from the joint posterior on the Poisson expected counts, $p\left(\Lambda_{0}, \Lambda_{1} \mid \vec{x}\right)$, where the set of triggers $\vec{x}$ have a chirp mass $\mathcal{M}>4.35 M_{\odot}$ and a FAR $<8766 \mathrm{yr}^{-1}$. The prior used to construct the joint counts posterior is taken to be the corresponding posterior from $\mathrm{O} 1$ and $\mathrm{O} 2$ [176].

\section{ESTIMATION OF SOURCE PARAMETERS}

Once triggers of interest are identified, the physical parameters of the candidate event gravitational-wave signals are inferred by computing their posterior probability density functions. The uncertainty in the source parameters is quantified by the posterior probability distribution $p(\vec{\vartheta} \mid \vec{d})$, which is calculated using Bayes' theorem as

$$
p(\vec{\vartheta} \mid \vec{d}) \propto p(\vec{d} \mid \vec{\vartheta}) \pi(\vec{\vartheta})
$$

where $p(\vec{d} \mid \vec{\vartheta})$ is the likelihood of the data given the model parameters $\vec{\vartheta}$ and $\pi(\vec{\vartheta})$ is the prior probability distribution for the parameters. The likelihood is calculated from a coherent analysis of data from each of the detectors. As in our previous analyses, e.g., Ref. [177], we assume that the noise can be treated as Gaussian, stationary, and uncorrelated between detectors $[63,178]$ in the stretch of data used to calculate the likelihood and to measure the noise PSD. This yields a Gaussian likelihood [143,179] for the data from a single detector:

$$
p\left(d^{i} \mid \vec{\vartheta}\right) \propto \exp \left[-\frac{1}{2}\left\langle d^{i}-h_{M}^{i}(\vec{\vartheta}) \mid d^{i}-h_{M}^{i}(\vec{\vartheta})\right\rangle\right],
$$

where $d^{i}$ is the data of the $i$ th instrument and $h_{M}^{i}(\vec{\vartheta})$ is the waveform model calculated at $\vec{\vartheta}$ projected on the $i$ th detector and adjusted to account for the uncertainty in offline calibration described in Sec. III A. The noiseweighted inner product $\langle a \mid b\rangle[143,180]$ requires specifying the frequency range in which the analysis is performed as well as the noise PSD.

Upon detection of a binary merger, exploratory analyses are first conducted to identify which models and settings are most suitable for use in our production analyses. In general, we use a low-frequency cutoff of $f_{\text {low }}=20 \mathrm{~Hz}$, 
unless data quality requirements at the time of specific candidate events are different, in which case a specific range is noted in Sec. VII. The high-frequency cutoff is always equal to the Nyquist frequency of the analysis, which is chosen on a per-event basis to be greater than the highest frequency of the $\ell=m=2$ mode, since the signals (especially the higher-mass $\mathrm{BBH}$ signals) do not require the full bandwidth available at the native sampling rate of $16 \mathrm{kHz}$. The PSD characterizing the noise at the time of each event is measured by BW using the same data that are used for likelihood computation [181,182]. We then obtain the final joint likelihood over all detectors by multiplying together the likelihood from each detector in Eq. (5).

The gravitational-wave signal emitted by a circularized compact binary composed of two black holes depends on 15 unknown parameters, defined in Sec. IV B. The initial masses and spins of the inspiraling black holes determine the peak gravitational-wave luminosity and mass and spin of the postmerger remnant black hole, which we calculate from fits to numerical relativity (NR) [5,183-187]. When one or both objects are neutron stars, matter effects modify the binary inspiral and are included via the dimensionless quadrupole tidal deformability $\Lambda_{i}$, adding one extra parameter for each neutron star in the binary. Other matter effects, such as octupolar and higher tidal deformabilities, nonblack hole spin-induced multipole moments, and $f$-mode resonances, are parameterized by the $\Lambda_{i}$ using quasiuniversal relations [188]. The dominant tidal contribution to the waveform is given by the dimensionless tidal deformability parameter $[189,190]$

$\tilde{\Lambda}=\frac{16}{13} \frac{\left[\left(m_{1}+12 m_{2}\right) m_{1}^{4} \Lambda_{1}+\left(m_{2}+12 m_{1}\right) m_{2}^{4} \Lambda_{2}\right]}{\left(m_{1}+m_{2}\right)^{5}}$.

Nonspinning black holes have $\Lambda_{i}=0$ [191,192], and the waveform models we use adopt the convention that this result is true for all black holes [193,194]. We do not calculate final masses and spins when matter effects are included in the analyses, as this calculation requires an accurate prediction of the ejected mass following possible tidal disruption, taking into account the equation of state and uncertain details of the dynamics of the merger.

\section{A. Waveform models}

We characterize the detected binaries using multiple waveform models, each of which uses a different set of modeling techniques and includes different physical effects. For every event with inferred component masses above $3 M_{\odot}$ in preliminary analyses, we perform production parameter estimation runs using a subset of $\mathrm{BBH}$ waveforms. IMRPhenomPv2 [195-197] is a phenomenological model for gravitational waves from precessing $\mathrm{BBH}$ systems, calibrated to NR and using an effective single-spin description to model effects from spin precession [198].
SEOBNRv4P $[157,199]$ is based on the effective-one-body (EOB) formalism [200,201] and calibrated to NR, with a generic two-spin treatment of the precession dynamics. These models rely on twisting-up procedures, where aligned-spin, NR-calibrated waveform models defined in the coprecessing frame are mapped (through a suitable frame rotation) to approximate the multipoles of a precessing system in the inertial frame [202-206]. These models do not include contributions to the strain from spherical harmonic modes beyond $\ell=2$, so we also analyze each event with at least one of the following models that incorporate higherorder multipole (HM) moments and precession effects: IMRPhenomPv3HM [207,208], SEOBNRv4PHM [199,209], and NRSur7dq4 [210]. IMRPhenomPv3HM (based on IMRPhenomHM [211]) and SEOBNRv4PHM (based on SEOBNRv4HM [212]) both rely on the twisting-up approach described above. NRSur7dq4 is a surrogate waveform model for BBH systems that directly interpolates a large set of precessing NR simulations. Unlike the other two HM models, NRSur7dq4 waveforms are restricted by the length of the NR simulations in the training set, covering only approximately 20 orbits before merger.

Any sources with evidence for at least one binary component below $3 M_{\odot}$ are characterized using several waveforms capable of modeling matter effects. For the BNS system GW190425 [32], we use the following: IMRPhenomD_NRTidal and IMRPhenomPv2_NRTidal [213,214], which are based on the BBH models IMRPhenomD and IMRPhenomPv2, respectively, and incorporate NR and tidal EOB-tuned contributions from tidal interaction as well as equation-of-state-dependent self-spin effects; TaylorF2 $[139,147-156]$, which describes waveforms from the inspiral of nonprecessing compact binaries, with matter effects derived in the post-Newtonian formalism, including quadrupole-monopole coupling parameterized in terms of the tidal deformabilities [188,215,216]; TEOBResums [217], an aligned-spin EOB model that incorporates post-Newtonian and self-force contributions to the tidal potential; and, finally, a frequency-domain surrogate model of aligned-spin SEOBNRv4T waveforms [157,218-220], which are derived in the EOB approach and include dynamical tides.

For potential NSBH sources with $m_{1}>3 M_{\odot}>m_{2}$, we use both $\mathrm{BBH}$ waveforms and the NSBH-specific alignedspin waveform models SEOBNRv4_ROM_NRTidalv2_NSBH [221] and IMRPhenomNSBH [222], which feature the dominant quadrupole modes. SEOBNRv4_ROM_NRTidalv2_NSBH uses SEOBNRv4_ROM as the BBH baseline, while IMRPhenomNSBH employs phase evolution from IMRPhenomD [195,196] and amplitude from IMRPhenomC [223]. Both models contain a phenomenological description of the tidal effects tuned to NR simulations [224] and include corrections to the amplitude through inspiral, merger, and ringdown to account for a possibility of tidal disruption.

To account for the systematic uncertainties in the waveform models, we combine equal numbers of posterior 
TABLE III. Waveform models used in this paper. We indicate which multipoles are included for each model. For precessing models, the multipoles correspond to those in the coprecessing frame. The combined key column specifies which results generated with these waveforms are combined in our data release under a common key. The models below the horizontal line include matter effects.

\begin{tabular}{|c|c|c|c|c|}
\hline Combined key & Waveform name & Precession & Multipoles $(\ell,|m|)$ & Refs. \\
\hline ZeroSpinIMR $^{\mathrm{a}}$ & IMRPhenomD & $\times$ & $(2,2)$ & {$[195,196]$} \\
\hline AlignedSpinIMR & SEOBNRv4_ROM & $\times$ & $(2,2)$ & [157] \\
\hline \multirow[t]{2}{*}{ AlignedSpinIMRHM } & IMRPhenomHM & $\times$ & $(2,2),(2,1),(3,3),(3,2),(4,4),(4,3)$ & [211] \\
\hline & SEOBNRv4HM_ROM & $\times$ & $(2,2),(2,1),(3,3),(4,4),(5,5)$ & {$[212,226]$} \\
\hline \multirow[t]{2}{*}{ PrecessingSpinIMR } & SEOBNRv4P & $\checkmark$ & $(2,2),(2,1)$ & {$[199,209,227]$} \\
\hline & IMRPhenomPv2 & $\checkmark$ & $(2,2)$ & {$[197,207]$} \\
\hline \multirow[t]{3}{*}{ PrecessingSpinIMRHM } & IMRPhenomPv3HM & $\checkmark$ & $(2,2),(2,1),(3,3),(3,2),(4,4),(4,3)$ & [208] \\
\hline & NRSur7dq4 & $\checkmark$ & $\ell \leq 4$ & [210] \\
\hline & SEOBNRv4PHM & $\checkmark$ & $(2,2),(2,1),(3,3),(4,4),(5,5)$ & {$[199,209,227]$} \\
\hline \multirow[t]{3}{*}{ AlignedSpinTidal $^{\mathrm{b}}$} & IMRPhenomD_NRTidal & $x$ & $(2,2)$ & {$[213,214]$} \\
\hline & TEOBResums & $x$ & $(2,2)$ & [217] \\
\hline & SEOBNRv4T_surrogate & $x$ & $(2,2)$ & {$[157,218-220]$} \\
\hline PrecessingSpinIMRTidal $^{\mathrm{b}}$ & IMRPhenomPv2_NRTidal & $\checkmark$ & $(2,2)$ & {$[213,214]$} \\
\hline AlignedSpinInspiralTidal $^{\mathrm{b}}$ & TaylorF2 & $x$ & $(2,2)$ & {$[139,147-156]$} \\
\hline \multirow[t]{2}{*}{ AlignedSpinIMRTidal_NSBH } & SEOBNRv4_ROM_NRTidalv2_NSBH & $x$ & $(2,2)$ & [221] \\
\hline & IMRPhenomNSBH & $x$ & $(2,2)$ & [222] \\
\hline
\end{tabular}

\footnotetext{
${ }^{\mathrm{a}}$ For these datasets, we enforce $\left|\vec{\chi}_{i}\right|=0$.

${ }^{\mathrm{b}}$ The data release contains versions of these keys with HS and LS in the name, which correspond to the high-spin $\left(\left|\vec{\chi}_{i}\right| \leq 0.89\right)$ and low-spin $\left(\left|\vec{\chi}_{i}\right| \leq 0.05\right)$ priors, respectively.
}

samples (described in Sec. VB) from all parameter estimation runs for an event that use waveforms with comparable physics. This event treats the constituent waveform models in the combined results as having equal weight rather than weighting them by marginal likelihood as suggested in Ref. [225]. Table III shows the waveforms employed in this work, the keys under which we group results using these waveforms, and descriptions of the physical effects incorporated in the models.

\section{B. Sampling methods}

We use several methods to draw samples from the posterior distributions on source parameters using the models described above. The LALInference [179] package is used for most analyses presented in this paper. This package provides two independent stochastic sampling algorithms: a MCMC algorithm and a nested sampling [228] algorithm. We employ LALInference 's nested sampling algorithm for most of the $\mathrm{BBH}$ analyses performed with the IMRPhenomD and IMRPhenomPv2 waveforms and the MCMC algorithm for those performed with SEOBNRv4P. However, the serial nature of these methods makes them unsuitable for use with some of the more computationally costly waveform models, such as waveforms with $\mathrm{HMs}$ and precession effects, especially for long-duration signals. For these, we also use RIFT, which performs a hybrid exploration of the parameter space split into intrinsic and extrinsic parameters [229-231], and Parallel Bilby, based on a distributed implementation of nested sampling [232-235], which is also used in previously published analyses of GW190412 [236], GW190425 [32], and GW190814 [33]. The raw posterior samples from the analyses described above are then collated to a common format using the PESummary package [237].

\section{Priors}

Each event is analyzed independently using a prior distribution on the source parameters that is chosen to ensure adequate sampling of the parameter space and simplicity in using the posterior samples for further analyses. We choose a prior that is uniform in spin magnitudes and redshifted component masses and isotropic in spin orientations, sky location, and binary orientation. The prior on luminosity distance corresponds to a uniform merger rate in the comoving frame of the source, using a flat $\Lambda \mathrm{CDM}$ cosmology with Hubble constant $H_{0}=67.9 \mathrm{~km} \mathrm{~s}^{-1} \mathrm{Mpc}^{-1}$ and matter density $\Omega_{m}=0.3065$ [238]; this physically motivated prior differs from that used in previous published results, which uses a prior $\propto D_{L}{ }^{2}$. However, our data release includes parameter estimation samples for both the flat-in-comoving-volume and $\propto D_{L}^{2}$ priors. For details on the conversion, see Appendix C. Intrinsic source masses are computed by dividing the redshifted masses measured in the detector frame by $(1+z)$, where $z$ is calculated using the same cosmological model.

For the LALInference and Parallel Bilby analyses, we marginalize over uncertainty in the strain calibration. The calibration errors in amplitude and phase are described by frequency-dependent splines, whose coefficients are allowed to vary alongside signal parameters in the inference. The prior distribution on the calibration error at each 
spline node is set by the measured uncertainty at each node [177].

\section{CANDIDATE EVENT LIST}

Table IV presents the results from each of cWB, GstLAL, and $\mathrm{PyCBC}$ passing a FAR threshold of $2.0 \mathrm{yr}^{-1}$; the full gravitational-wave name encodes the UTC date with the time of the event given after the underscore. GW190521 [34,35], GW190425 [32], GW190412 [236], and GW190814 [33] were published previously, and these names are used here verbatim. The $2.0 \mathrm{yr}^{-1}$ threshold is chosen to be higher (more permissive) than the threshold used for public alerts, $1.2 \mathrm{yr}^{-1}$ [239], but sufficiently low to provide an expected contamination fraction below $10 \%$. An extended candidate event list, which may contain marginally significant triggers, will be provided later once final data calibration and quality checks are available. Unlike GWTC-1 [8], a separate $p_{\text {astro }}$ threshold is not applied; however, $p_{\text {astro }}$ is greater than $50 \%$ for all candidate events for which $p_{\text {astro }}$ is calculated in this work, satisfying the same criteria as GWTC-1 [240]. Among the 39 reported candidate events passing the FAR threshold of $2.0 \mathrm{yr}^{-1}$, 15 are detected by cWB, 36 candidate events are detected by GstLAL, and 27 candidate events are detected by РуСBC; 25 candidate events are recovered by at least two pipelines. Given the FAR threshold and number of candidate events detected, the expectation value for noise events is $<4$ in this list. Based on the FAR or the probability of being a signal described in Sec. IV D, GW190426_152155, GW190719_215514, and GW190909_114149 are the most likely to be noise among the candidate event list.

cWB recovers fewer candidate events than either GstLAL or PyCBC. This result is expected, because cWB has the highest sensitivity for short-duration, high-mass signals, and its sensitivity decreases for lower-mass systems with longer duration. cWB also requires candidate events to be found in coincidence between at least two detectors.

The difference in candidate event recovery between GstLAL and $\mathrm{PyCBC}$ is primarily due to $\mathrm{PyCBC}$ analyzing only times when both LIGO Hanford and LIGO Livingston are operating and requiring signals to be observed in both. РусвС does not analyze Virgo data due to the fact that the code version used for this catalog had not fully integrated three-detector analysis including Virgo. GstLAL analyzes LIGO and Virgo data and allows for the detection of candidate events from one, two, or three gravitationalwave detectors. These algorithmic choices account for the GstLAL-only detection of GW190424_180648, GW190425, GW190620_030421, GW190708_232457, and GW190910_112807, which are detected above the required SNR threshold only in the LIGO Livingston detector, and for GW190630_185205, GW190701_ 203306, and GW190814, where the inclusion of Virgo is essential for determining event significance. The difference in candidate event recovery between $\mathrm{PyCBC}$ and GstLAL is also consistent with the results of the simulation presented in Sec. IV C. After accounting for differences in analyzed data, the GstLAL and $\mathrm{PyCBC}$ methods detect a comparable number of candidate events.

The remaining differences between the candidate event lists from GstLAL (GW190426_152155, GW190527_ 092055, GW190909_114149, and GW190929_012149) and РyCBC (GW190413_052954, GW190514_065416, and GW190719_215514) arise from the low SNR of each event (SNR $\lesssim 10)$. Small fluctuations in SNR caused by different PSD estimation and data segmentation between pipelines lead to differences in significance estimation. This list of low SNR candidate events, which are identified by only one pipeline, also contains the three candidate events with the highest minimum FAR among the pipelines (GW190426_152155, GW190719_215514, and GW190909_114149) which have the highest likelihood among the full candidate event list of being caused by noise.

Since 2 April 2019 20:00 UTC, the LVC produced automated, public preliminary GCN notices for gravitational-wave candidate events appearing in two or more interferometers with FARs less than 6 per year before a multiple analysis trials factor was applied, resulting in an effective threshold of $1.2 \mathrm{yr}^{-1}$ [248]. On 11 June 2019, this threshold was extended to include gravitational-wave candidate events appearing in only one interferometer and satisfying the same FAR threshold. During O3a, 33 candidate events are disseminated as plausible astrophysical signals; seven are not recovered above the threshold considered in this work [249].

S190510g, S190718y, S190901ap, S190910d, S190910h, S190923y, and S190930t [241-247] are the seven candidate events disseminated via GCNs which are not recovered here. S190718y, S190901ap, S190910h, and S190930t are initially identified as single-detector candidate events (with an SNR above threshold in only one detector) with FARs of $1.14,0.22,1.14$, and $0.47 \mathrm{yr}^{-1}$, respectively. Relaxing the demand for coincident observation across interferometers allows LVC analyses to report on additional astrophysically interesting candidate events $[172,250]$ but also removes a powerful check on the search background and leads to larger uncertainties in the FAR. All public alerts are subsequently followed up in low latency to assess whether the analysis pipelines and detectors are operating as expected. The low-latency follow-up of S190718y, S190901ap, S190910h, and S190930t does not uncover any reason to retract these candidate events based on data quality. However, after offline reanalysis with additional background statistics, these four single-detector candidate events are no longer significant enough to merit inclusion in Table IV.

The remaining three public alert candidate events not recovered here-S190510g, S190910d, and S190923yare found in coincidence in low latency, albeit at modest 
TABLE IV. Gravitational-wave candidate event list. We find 39 candidate events passing the FAR threshold of $2.0 \mathrm{yr}^{-1}$ in at least one of the four searches. Except for previously published events, the gravitational-wave name encodes the UTC date with the time of the event given after the underscore. Bold-faced names indicate the events that were not previously reported. The second column denotes the observing instruments. For each of the four pipelines, cWB, GstLAL, PyCBC, and PyCBC BBH, we provide the FAR and network SNR. Of the 39 candidate events, the five that are found above the required SNR threshold in only one of the gravitational-wave detectors are denoted by a dagger $(\dagger)$. For candidate events found above threshold in only one detector (single-detector candidate events), the FAR estimate involves extrapolation. All single-detector candidate events in this list by definition are rarer than the background data collected in this analysis. Therefore, a conservative bound on the FAR for triggers denoted by $\dagger$ is approximately $2 \mathrm{yr}^{-1}$. GW190521, GW190602_175927, GW190701_203306, and GW190706_222641 are identified by the cWB high-mass search as described in Sec. IVA. GstLAL FARs are capped at $1 \times 10^{-5} \mathrm{yr}^{-1}$ to be consistent with the limiting FARs from other pipelines. $\cdots$ indicate that a pipeline does not find the event below the specified $2.0 \mathrm{yr}^{-1}$ threshold. Blank entries indicate that the data are not searched by a pipeline. The probability that an event is astrophysical in origin as described in Sec. IV D is indicated in the column $p_{\text {astro }}$.

\begin{tabular}{|c|c|c|c|c|c|c|c|c|c|c|c|c|}
\hline \multirow[t]{2}{*}{ Name } & \multirow[t]{2}{*}{ Instrument } & \multicolumn{2}{|l|}{$\mathrm{cWB}$} & \multicolumn{3}{|c|}{ GstLAL } & \multicolumn{3}{|c|}{ РусвС } & \multicolumn{3}{|c|}{ РусвС ВBН } \\
\hline & & FAR $\left(\mathrm{yr}^{-1}\right)$ & $\mathrm{SNR}^{\mathrm{a}}$ & FAR $\left(\mathrm{yr}^{-1}\right)$ & SNR & $p_{\text {astro }}$ & FAR $\left(\mathrm{yr}^{-1}\right)$ & $\mathrm{SNR}^{\mathrm{a}}$ & $p_{\text {astro }}$ & FAR $\left(\mathrm{yr}^{-1}\right)$ & $\mathrm{SNR}^{\mathrm{a}}$ & $p_{\text {astro }}$ \\
\hline GW190408_181802 & HLV & $<9.5 \times 10^{-4}$ & 14.8 & $<1.0 \times 10^{-5}$ & 14.7 & 1.00 & $<2.5 \times 10^{-5}$ & 13.5 & 1.00 & $<7.9 \times 10^{-5}$ & 13.6 & 1.00 \\
\hline GW190412 & HLV & $<9.5 \times 10^{-4}$ & 19.7 & $<1.0 \times 10^{-5}$ & 18.9 & 1.00 & $3.1 \times 10^{-5}$ & 17.9 & 1.00 & $<7.9 \times 10^{-5}$ & 17.8 & 1.00 \\
\hline GW190413_052954 & HLV & $\ldots$ & $\ldots$ & $\ldots$ & $\ldots$ & $\ldots$ & $\ldots$ & $\ldots$ & $\ldots$ & $7.2 \times 10^{-2}$ & 8.6 & 0.98 \\
\hline GW190413_134308 & HLV & $\ldots$ & $\cdots$ & $3.8 \times 10^{-1}$ & 10.0 & 0.95 & $\ldots$ & $\cdots$ & $\cdots$ & $4.4 \times 10^{-2}$ & 9.0 & 0.98 \\
\hline GW190421_213856 & $\mathrm{HL}$ & $3.0 \times 10^{-1}$ & 9.3 & $7.7 \times 10^{-4}$ & 10.6 & 1.00 & $1.9 \times 10^{9}$ & 10.2 & 0.89 & $6.6 \times 10^{-3}$ & 10.2 & 1.00 \\
\hline GW190424_180648 & $\mathrm{L}$ & & & $7.8 \times 10^{-1 \dagger}$ & 10.0 & 0.91 & & & & & & \\
\hline GW190425 & LV & & & $7.5 \times 10^{-4^{\dagger}}$ & 13.0 & $\ldots$ & & & & & & \\
\hline GW190426_152155 & HLV & $\ldots$ & $\cdots$ & $1.4 \times 10^{0}$ & 10.1 & $\ldots$ & $\ldots$ & $\cdots$ & $\cdots$ & $\ldots$ & $\cdots$ & $\cdots$ \\
\hline GW190503_185404 & HLV & $1.8 \times 10^{-3}$ & 11.5 & $<1.0 \times 10^{-5}$ & 12.1 & 1.00 & $3.7 \times 10^{-2}$ & 12.2 & 1.00 & $<7.9 \times 10^{-5}$ & 12.2 & 1.00 \\
\hline GW190512_180714 & HLV & $8.8 \times 10^{-1}$ & 10.7 & $<1.0 \times 10^{-5}$ & 12.3 & 1.00 & $3.8 \times 10^{-5}$ & 12.2 & 1.00 & $<5.7 \times 10^{-5}$ & 12.2 & 1.00 \\
\hline GW190513_205428 & HLV & $\ldots$ & $\ldots$ & $<1.0 \times 10^{-5}$ & 12.3 & 1.00 & $3.7 \times 10^{-4}$ & 11.8 & 1.00 & $<5.7 \times 10^{-5}$ & 11.9 & 1.00 \\
\hline GW190514_065416 & HL & $\ldots$ & $\ldots$ & $\ldots$ & $\ldots$ & $\ldots$ & $\ldots$ & $\ldots$ & $\ldots$ & $5.3 \times 10^{-1}$ & 8.3 & 0.96 \\
\hline GW190517_055101 & HLV & $6.5 \times 10^{-3}$ & 10.7 & $9.6 \times 10^{-4}$ & 10.6 & 1.00 & $1.8 \times 10^{-2}$ & 10.4 & 1.00 & $<5.7 \times 10^{-5}$ & 10.2 & 1.00 \\
\hline GW190519_153544 & HLV & $3.1 \times 10^{-4}$ & 14.0 & $<1.0 \times 10^{-5}$ & 12.0 & 1.00 & $<1.8 \times 10^{-5}$ & 13.0 & 1.00 & $<5.7 \times 10^{-5}$ & 13.0 & 1.00 \\
\hline GW190521 & HLV & $2.0 \times 10^{-4}$ & 14.4 & $1.2 \times 10^{-3}$ & 15.0 & 1.00 & $1.1 \times 10^{0}$ & 12.6 & 0.93 & & $\ldots$ & $\ldots$ \\
\hline GW190521_074359 & $\mathrm{HL}$ & $<1.0 \times 10^{-4}$ & 24.7 & $<1.0 \times 10^{-5}$ & 24.4 & 1.00 & $<1.8 \times 10^{-5}$ & 24.0 & 1.00 & $<5.7 \times 10^{-5}$ & 24.0 & 1.00 \\
\hline GW190527_092055 & HL & $\ldots$ & $\ldots$ & $6.2 \times 10^{-2}$ & 8.9 & 0.99 & $\ldots$ & $\ldots$ & $\ldots$ & $\ldots$ & $\ldots$ & $\cdots$ \\
\hline GW190602_175927 & HLV & $1.5 \times 10^{-2}$ & 11.1 & $1.1 \times 10^{-5}$ & 12.1 & 1.00 & $\ldots$ & $\ldots$ & .. & $\ldots$ & $\ldots$ & $\ldots$ \\
\hline GW190620_030421 & LV & & & $2.9 \times 10^{-3^{\dagger}}$ & 13.1 & 1.00 & & & & & & \\
\hline GW190630_185205 & $\mathrm{LV}$ & & & $<1.0 \times 10^{-5^{\dagger}}$ & 15.6 & 1.00 & & & & & & \\
\hline GW190701_203306 & HLV & $5.5 \times 10^{-1}$ & 10.2 & $1.1 \times 10^{-2}$ & 11.6 & 1.00 & $\ldots$ & $\ldots$ & $\ldots$ & $\ldots$ & $\ldots$ & $\ldots$ \\
\hline GW190706_222641 & HLV & $<1.0 \times 10^{-3}$ & 12.7 & $<1.0 \times 10^{-5}$ & 12.3 & 1.00 & $6.7 \times 10^{-5}$ & 11.7 & 1.00 & $<4.6 \times 10^{-5}$ & 12.3 & 1.00 \\
\hline GW190707_093326 & HL & $\ldots$ & $\ldots$ & $<1.0 \times 10^{-5}$ & 13.0 & 1.00 & $<1.0 \times 10^{-5}$ & 12.8 & 1.00 & $<4.6 \times 10^{-5}$ & 12.8 & 1.00 \\
\hline GW190708_232457 & LV & & & $2.8 \times 10^{-5^{\dagger}}$ & 13.1 & 1.00 & & & & & & \\
\hline GW190719_215514 & HL & $\cdots$ & $\cdots$ & $\ldots$ & $\ldots$ & $\cdots$ & $\ldots$ & $\cdots$ & $\cdots$ & $1.6 \times 10^{0}$ & 8.0 & 0.82 \\
\hline GW190720_000836 & HLV & $\ldots$ & $\ldots$ & $<1.0 \times 10^{-5}$ & 11.7 & 1.00 & $<2.0 \times 10^{-5}$ & 10.6 & 1.00 & $<3.7 \times 10^{-5}$ & 10.5 & 1.00 \\
\hline GW190727_060333 & HLV & $8.8 \times 10^{-2}$ & 11.4 & $<1.0 \times 10^{-5}$ & 12.3 & 1.00 & $3.5 \times 10^{-3}$ & 11.5 & 1.00 & $<3.7 \times 10^{-5}$ & 11.8 & 1.00 \\
\hline GW190728_064510 & HLV & $\ldots$ & $\ldots$ & $<1.0 \times 10^{-5}$ & 13.6 & 1.00 & $<1.6 \times 10^{-5}$ & 13.4 & 1.00 & $<3.7 \times 10^{-5}$ & 13.4 & 1.00 \\
\hline GW190731_140936 & HL & $\cdots$ & $\cdots$ & $2.1 \times 10^{-1}$ & 8.5 & 0.97 & $\ldots$ & $\cdots$ & $\cdots$ & $2.8 \times 10^{-1}$ & 8.2 & 0.96 \\
\hline GW190803_022701 & HLV & $\ldots$ & $\cdots$ & $3.2 \times 10^{-2}$ & 9.0 & 0.99 & $\ldots$ & $\ldots$ & $\ldots$ & $2.7 \times 10^{-2}$ & 8.6 & 0.99 \\
\hline GW190814 & LV & & & $<1.0 \times 10^{-5}$ & 22.2 & 1.00 & & & & & & \\
\hline GW190828_063405 & HLV & $<9.6 \times 10^{-4}$ & 16.6 & $<1.0 \times 10^{-5}$ & 16.0 & 1.00 & $<1.5 \times 10^{-5}$ & 15.3 & 1.00 & $<3.3 \times 10^{-5}$ & 15.3 & 1.00 \\
\hline GW190828_065509 & HLV & $\ldots$ & $\ldots$ & $<1.0 \times 10^{-5}$ & 11.1 & 1.00 & $5.8 \times 10^{-5}$ & 10.8 & 1.00 & $<3.3 \times 10^{-5}$ & 10.8 & 1.00 \\
\hline GW190909_114149 & HL & $\ldots$ & $\cdots$ & $1.1 \times 10^{0}$ & 8.5 & 0.89 & $\ldots$ & $\ldots$ & $\ldots$ & $\ldots$ & $\ldots$ & $\ldots$ \\
\hline GW190910_112807 & $\mathrm{LV}$ & & & $1.9 \times 10^{-5^{\dagger}}$ & 13.4 & 1.00 & & & & & & \\
\hline GW190915_235702 & HLV & $<1.0 \times 10^{-3}$ & 12.3 & $<1.0 \times 10^{-5}$ & 13.1 & 1.00 & $8.6 \times 10^{-4}$ & 13.0 & 1.00 & $<3.3 \times 10^{-5}$ & 12.7 & 1.00 \\
\hline GW190924_021846 & HLV & $\ldots$ & $\cdots$ & $<1.0 \times 10^{-5}$ & 13.2 & 1.00 & $<6.3 \times 10^{-5}$ & 12.5 & 1.00 & $<3.3 \times 10^{-5}$ & 12.4 & 1.00 \\
\hline GW190929_012149 & HLV & $\ldots$ & $\ldots$ & $2.0 \times 10^{-2}$ & 9.9 & 1.00 & $\ldots$ & $\ldots$ & $\ldots$ & $\ldots$ & $\ldots$ & $\ldots$ \\
\hline GW190930_133541 & HL & $\ldots$ & $\cdots$ & $5.8 \times 10^{-1}$ & 10.0 & 0.92 & $3.4 \times 10^{-2}$ & 9.7 & 1.00 & $3.3 \times 10^{-2}$ & 9.8 & 0.99 \\
\hline
\end{tabular}

${ }^{a}$ PyCBC and cWB SNRs do not include Virgo. S190510g, S190718y, S190901ap, S190910d, S190910h, S190923y, and S190930t [241-247] are candidate events disseminated via GCNs which are not recovered here. The SNR for GW190814 (22.2) differs from the previously published value [33] because the non-observing-mode LIGO Hanford data are not analyzed in this work. The FAR of GW190425 differs from Ref. [32] due to different background data and pipeline configuration. The SNR of GW190521 differs from Ref. [34] due to the inclusion of subthreshold Virgo SNR. 
significance. S190510g is found in low latency by GstLAL and assigned a FAR of $0.28 \mathrm{yr}^{-1}$. There are initially data quality concerns with S190510g [251], and offline followup using an additional $24 \mathrm{~h}$ of background collection reveals the candidate event to be less significant than originally estimated [252]. Comparison to the full O3a background corroborates that the candidate event no longer passes the FAR threshold of $2.0 \mathrm{yr}^{-1}$. S190910d is identified in low latency by the SPIIR compact binary search pipeline [115] with a FAR of $0.12 \mathrm{yr}^{-1}$. The compact binary search pipeline MBTAOnline also records a lowsignificance candidate event at this time. However, the candidate event is not observed in low latency or offline by GstLAL, PyCBC, or cWB as significant. Presently, MBTAOnline and SPIIR are configured to run in low latency only. S190923y is reported in low latency by РуСВC and assigned a FAR of $1.51 \mathrm{yr}^{-1}$. GstLAL and MBTAOnline also record low-significance candidate events at this time. No pipeline retains this candidate event in the offline analysis below the threshold of $2.0 \mathrm{yr}^{-1}$, and it is therefore excluded from Table IV. These and other subthreshold events will be explored further in a future publication.

The remaining 26 public alerts are recovered in our offline analysis and included in the candidate event list presented in Table IV. The table also includes 13 gravitational-wave detections not previously reported. Four of these detections-GW190424_180648, GW190620_ 030421, GW190708_232457, and GW190910_112807have detection-level SNR $(\geq 4)$ in only one of the two LIGO detectors, making them single-detector observations from the perspective of candidate event significance. The incorporation of iDQ data quality into event ranking, combined with tuning of the signal consistency tests to further reject the O3a glitch background, improves the sensitivity of the offline GstLAL analysis to singledetector candidate events compared to the low-latency configuration, which accounts for these new discoveries. Nine new detections are observed in two or more interferometers and appear for the first time in Table IV: GW190413_052954, GW190413_134308, GW190514_ 065416, GW190527_092055, GW190719_215514, GW190731_140936, GW190803_022701, GW190909_ 114149, and GW190929_012149. Several of these detections are observed in low latency but do not meet the criteria for public release. They all exhibit moderate network SNRs $(\lesssim 10)$. The offline analyses here differ from their low-latency counterparts through having improved template banks, improved use of data quality information, improved data calibration, data cleaning, and improved tuning to reject the nonstationary noise background observed in O3a. These differences account for the new moderate SNR candidate events.

During O3a, the LVC issues eight retractions for public alerts that are promptly determined to be unlikely to have originated from astrophysical systems. The LIGO
Livingston detector is more problematic for low-latency analyses than in previous observing runs with the rate of noise glitches being significantly higher than in O2 (see Sec. III B). Low-latency detection, especially for candidate events originating in only one interferometer, is especially challenging during $\mathrm{O} 3 \mathrm{a}$.

S190405ar is the first retraction [253]. This notice is distributed in error and is never considered to be of astrophysical origin, because the event's FAR is significantly above threshold at $6800 \mathrm{yr}^{-1}$. The remaining retractions are caused by severely nonstationary noise. A glitch in LIGO Hanford data leads to the identification and subsequent retraction of S190518bb [254]. S190524q, S190808ae, S190816i, S190822c, S190829u, and S190928c all exhibit extreme nonstationary noise in the LIGO Livingston detector [255-260]. S190822c is assigned a significant FAR at identification but is observed only in a single interferometer. As previously mentioned, single-detector triggers are subject to higher uncertainties in FAR. Out of these triggers, an automatic gating method deployed later in the observing run used by searches in order to mitigate nonstationary noise in the detectors would have vetoed S190822c. However, at the time, this automatic gating was not being employed. FARs calculated by the pipelines are correct if the data collected so far are representative of the future data. Online pipelines assign FARs using previously collected data, while offline analyses have access to asynchronous background. If a new nonstationary noise source emerges in the data during an observing run, it is possible for it to be misidentified as a candidate event. Although this misidentification can also affect candidates observed in multiple interferometers, single-detector candidates are especially susceptible to novel noise sources seen for the first time in low latency. In some cases where the impact of glitches is unknown, follow-up analyses are performed to remove instrumental artifacts and reassess the candidate event significance. After follow-up, none of these eight retracted candidate events remain significant. Subsequent offline analyses do not identify them as significant, either.

\section{SOURCE PROPERTIES}

We analyze the 39 candidate events shown in Table IV with the parameter estimation techniques described in Sec. V. For a subset of candidate events, event validation procedures outlined in Sec. III C identify transient noise that may impact the results of parameter inference. In order to minimize the effect of this transient noise, candidatespecific procedures are explored for all impacted candidate events. In most cases, transient noise is mitigated through the glitch-subtraction methods outlined in Sec. III D. After application of these methods, the identified transient noise is considered mitigated if the data surrounding the event are consistent with Gaussian noise, as measured by the variance of the measured power spectral density during the time period containing the identified transient [97]. If data 
TABLE V. List of candidate-specific data usage and mitigation methods for parameter estimates. Only candidate events for which mitigation of instrumental artifacts is performed are listed. The glitch-subtraction methods used for these candidate events are detailed in Sec. III D. The minimum frequency is the lower limit of data used in analyses of gravitational-wave source properties for the listed interferometer.

\begin{tabular}{ll}
\hline \hline Name & Mitigation \\
\hline GW190413_134308 & L1 glitch subtraction, glitch-only model \\
GW190424_180648 & L1 glitch subtraction, glitch-only model \\
GW190425 & L1 glitch subtraction, glitch-only model \\
GW190503_185404 & L1 glitch subtraction, glitch-only model \\
GW190513_205428 & L1 glitch subtraction, glitch-only model \\
GW190514_065416 & L1 glitch subtraction, glitch-only model \\
GW190701_203306 & L1 glitch subtraction, glitch + signal \\
& model \\
GW190727_060333 & L1 $f_{\text {min }}: 50$ Hz \\
GW190814 & L1 $f_{\text {min }}: 30$ Hz; H1 nonobserving data \\
GW190924_021846 & L1 glitch subtraction, glitch-only model \\
\hline \hline
\end{tabular}

are not Gaussian after glitch subtraction, we evaluate the SNR lost by restricting the frequency range of data considered in parameter inference to fully excise the identified transient noise. In cases where the single-detector SNR loss is below $10 \%$, this reduced frequency range is used in analyses. Otherwise, the nominal frequency range is used. The full list of candidate events using candidatespecific mitigation, along with the mitigation configuration, is found in Table V.

Based on the investigations described in Appendix A, we find that most gravitational-wave candidate events in this catalog exhibit small changes in source parameter estimates when spherical harmonic modes above $\ell=2$ are included. However, these differences in aggregate could still affect population-level studies, so we present as fiducial results the combined posterior samples of HM runs for all BBHs candidate events except GW190707_093326, GW190720_ 000836, GW190728_064510, GW190915_235702, GW190924_021846, and GW190930_133541. For these six exceptions, we present combined IMRPhenomPv2SEOBNRv4P samples, because the effect of higher modes is either negligible or subdominant to the systematics between IMRPhenomPv2 and SEOBNRv4P results, as detailed in Appendix A. GW190412 [236], GW190521 [34,35], and GW190814 [33] are analyzed extensively in separate publications with the HM waveform families SEOBNRv4PHM, IMRPhenOMPv3HM, Or NRSur7dq4, so we present HM runs for these candidate events as fiducial results here and defer readers to those publications for details on those candidate events [261].

GW190425, GW190426_152155, and GW190814 show indications of including at least one neutron star, and so are also analyzed using tidal waveforms in addition to IMRPhenomPv2 and SEOBNRv4P, and are discussed in
Sec. VII B. Further details on waveform systematics and the waveforms employed in this work can be found in Appendix A, and the full suite of posterior samples is publicly available [262].

In the following subsections, we summarize the results of our parameter estimation analyses and highlight candidate events of particular interest. To identify candidate events with the most extreme parameter values, we repeatedly select one posterior sample at random from each event and record which candidate events have the lowest and highest values of each parameter. From these repeated trials, we determine each event's probability of having the lowest or highest value for a given parameter. Table VI shows $90 \%$ credible intervals on the source parameters of all 39 candidate events using the priors described in Sec. V C and waveforms specified above.

To provide an overview of the posterior distributions of the source parameters for all GWTC- 2 candidate events, we show $90 \%$ credible regions for all candidate events in the $M-q$ and $\mathcal{M}-\chi_{\text {eff }}$ planes in Figs. 6 and 7, respectively, and the corresponding one-dimensional marginal posterior distributions on $m_{1}, q$, and $\chi_{\text {eff }}$ in Fig. 8.

\section{A. Masses of sources with $\boldsymbol{m}_{\mathbf{2}}>\mathbf{3} \boldsymbol{M}_{\odot}$}

Our candidate event list includes compact binary mergers that have higher total masses than those in GWTC-1 [8] as well as mergers with component masses in the purported lower mass gap of approximately 2.5-5 $M_{\odot}$ [263-266]. Here, we describe the masses for candidate events with $m_{2}>3 M_{\odot}$, which we can confidently expect to be BBHs. The remaining candidate events are described separately in Sec. VII B.

A majority of the masses of black holes reported herein are larger than those reported via electromagnetic observations [267-269]. By repeatedly selecting one posterior sample at random from each event and recording the most massive among the ensemble, we find that the most massive binary system is probably the one associated with GW190521 [34,35]. This system has a $98 \%$ probability of being the most massive, with a total mass of $163.9_{-23.5}^{+39.2} M_{\odot}$ and remnant mass $15.6_{-22.4}^{+36.8} M_{\odot}$, where we average over SEOBNRv4PHM, NRSur7dq4, and IMRPhenomPv3HM waveform families. This averaging is done for consistency with other sources contained in this catalog, in contrast to the individual results reported in Ref. [35], where the NRSur7dq4 results are highlighted. The more massive component in the source of GW190521 has a $66 \%$ probability of being the most massive $\mathrm{BH}$ detected in gravitational waves to date $\left(m_{1}=95.3_{-18.9}^{+28.7} M_{\odot}\right)$. GW190519_153544, GW190602_ 175927, and GW190706_222641 also have notably high total masses with over $50 \%$ posterior support for total mass $M>100 M_{\odot}$.

The least massive O3a system with $m_{2}>3 M_{\odot}$ is probably $(96 \%)$ the one associated with GW190924_021846 
TABLE VI. Median and $90 \%$ symmetric credible intervals on selected source parameters. The columns show source total mass $M$, chirp mass $\mathcal{M}$ and component masses $m_{i}$, dimensionless effective inspiral spin $\chi_{\text {eff }}$, luminosity distance $D_{L}$, redshift $z$, final mass $M_{f}$, final spin $\chi_{f}$, and sky localization $\Delta \Omega$. The sky localization is the area of the $90 \%$ credible region. For GW190425, we show the results using the high-spin prior $\left(\left|\vec{\chi}_{i}\right| \leq 0.89\right)$. We also report the network matched-filter SNR for all events. These SNRs are from LALInference IMRPhenomPv2 runs, since RIFT does not produce the SNRs automatically, except for GW190425 and GW190426_152155, which use the SNRs from fiducial runs, and GW190412, GW190521, and GW190814, which use IMRPhenomPv3HM SNRs. For GW190521, we report results averaged over three waveform families, in contrast to the results highlighting one waveform family in Ref. [34].

\begin{tabular}{|c|c|c|c|c|c|c|c|c|c|c|c|}
\hline vent & $M\left(M_{\odot}\right)$ & $1\left(M_{\odot}\right)$ & $m_{1}\left(M_{\odot}\right)$ & $m_{2}\left(M_{\odot}\right)$ & $\chi_{\text {eff }}$ & $\mathrm{O}_{L}(\mathrm{Gpc})$ & $z$ & $M_{f}\left(M_{\odot}\right)$ & $\chi_{f}$ & $\left(\operatorname{deg}^{2}\right)$ & NR \\
\hline & & & & & & & & & & 150 & \\
\hline W190412 & & $3.3_{-0.3}^{+0.4}$ & $30.1_{-5.1}^{+4.7}$ & & & & & & & 21 & $18.9_{-0.3}^{+0.2}$ \\
\hline & $58.6_{-9.7}^{+13.3}$ & $24.6_{-4.1}^{+5.5}$ & $34.7_{-8.1}^{+12.6}$ & $23.7_{-6.7}^{+7.3}$ & $-0.01_{-0.34}^{+0.29}$ & $3.55_{-1.66}^{+2.27}$ & $0.59_{-0.24}^{+0.29}$ & $56.0_{-9.2}^{+12.5}$ & $0.68_{-}^{+}$ & 1500 & $8.9_{-0.7}^{+0.4}$ \\
\hline & & & & $3.18_{-10.8}^{+11.7}$ & $-0.03_{-0.29}^{+0.25}$ & $4.45_{-2.12}^{+2.48}$ & $0.71_{-0.30}^{+0.31}$ & $75.5_{-11.4}^{+16.4}$ & $0.68_{-}^{+}$ & 730 & $10.0_{-0.5}^{+0.4}$ \\
\hline & $72.9_{-9.2}^{+13.4}$ & $1.2_{-4.2}^{+5.9}$ & $41.3_{-6.9}^{+10.4}$ & $31.9_{-8.8}^{+8.0}$ & $-0.06_{-0.27}^{+0.22}$ & $2.88_{-1.38}^{+1.37}$ & $0.49_{-0.21}^{+0.19}$ & & & 1200 & $10.7_{-0.4}^{+0.2}$ \\
\hline & $\begin{array}{l}+13.3 \\
-10.7\end{array}$ & $1.0_{-4.6}^{+5.8}$ & $40.5_{-7.3}^{+11.1}$ & & $0.13_{-0.22}^{+0.22}$ & $2.20_{-1.16}^{+1.58}$ & & $68.9_{-10.1}^{+12.4}$ & $0.74_{-0.09}^{+0.09}$ & 28000 & $10.4_{-0.4}^{+0.2}$ \\
\hline & & $.44_{-0.02}^{+0.02}$ & & & $0.06_{-}^{+}$ & $\begin{array}{l}.07 \\
07\end{array}$ & $0.03_{-}^{+}$ & & $\ldots$ & 10000 & \\
\hline & & $.41_{-0.08}^{+0.08}$ & & & $-0.03_{-0.30}^{+0.32}$ & & & & $\ldots$ & 1300 & \\
\hline 404 & $.7_{-8.3}^{+9.4}$ & $2_{-4.2}^{+4.2}$ & & $28.4_{-8.0}^{+7.7}$ & $-0.03_{-}^{+}$ & $1.45^{+}$ & & & $0.66_{-0.12}^{+0.09}$ & 94 & $12.4_{-0.3}^{+0.2}$ \\
\hline & & $14.6_{-1.0}^{+1.3}$ & & $12.6_{-2.5}^{+3.6}$ & $0.03^{+}$ & & & & & 220 & \\
\hline 428 & & $1.6_{-1.9}^{+3.8}$ & & $18.0_{-4.1}^{+7.7}$ & $0.11_{-}^{+}$ & $2.06_{-}^{+}$ & & $51.6_{-5.8}^{+8.2}$ & & 520 & \\
\hline & & $28.5_{-4.8}^{+7.9}$ & $39.0_{-8.2}^{+14.7}$ & & -0.1 & & & & & 3000 & \\
\hline & & $26.6_{-4.0}^{+4.0}$ & $37.4_{-7.6}^{+11.7}$ & $25.3_{-7.3}^{+7.0}$ & $0.52^{+}$ & $.86_{-0.84}^{+1.62}$ & & & & 470 & \\
\hline & $\begin{array}{r}+13.5 \\
-14.8\end{array}$ & $44.5_{-7.1}^{+6.4}$ & & $40.5_{-11.1}^{+11.0}$ & & & $0.44_{-}^{+}$ & & & 860 & $15.6_{-0.3}^{+0.2}$ \\
\hline & & & & $\begin{array}{r}+22.7 \\
-23.1\end{array}$ & & & & 156.3 & & 1000 & \\
\hline 59 & $74.7_{-4.8}^{+7.0}$ & $32.1_{-2.5}^{+3.2}$ & $42.2_{-4.8}^{+5.9}$ & $32.8_{-6.4}^{+5.4}$ & $0.09_{-}^{+}$ & & & & & 550 & \\
\hline & & $24.3_{-4.2}^{+9.1}$ & $36.5_{-9.0}^{+16.4}$ & $22.6_{-8.1}^{+10.5}$ & & & & & & 3700 & \\
\hline & & & & & & & & & & 690 & \\
\hline & $92.1_{-13.1}^{+18.5}$ & $38.3_{-6.5}^{+8.3}$ & $57.1_{-12.7}^{+16.0}$ & $35.5_{-12.3}^{+12.2}$ & 0.33 & & 0.49 & 87.2 & & 7200 & \\
\hline & & $24.9_{-2.1}^{+2.1}$ & & & & & & & & 1200 & $15.6_{-0.3}^{+0.2}$ \\
\hline 306 & & & & & & & & & & 46 & \\
\hline & & & $67.0_{-16.2}^{+14.6}$ & $38.2_{-13.3}^{+14.6}$ & $0.28_{-}^{+}$ & & & & & 650 & \\
\hline & & & & & & & & & & 00 & \\
\hline 32457 & & & & & & & & & & 14000 & \\
\hline & & $23.5_{-4.0}^{+6.5}$ & $36.5_{-10.3}^{+18.0}$ & & & & & & & 2900 & \\
\hline 36 & & & & & & & & & & 460 & $11.0_{-0.7}^{+0.3}$ \\
\hline W190727_060333 & & & & & & & & & & 830 & \\
\hline & $20.6_{-1.3}^{+4.5}$ & & & & & & & $19.6_{-1.3}^{+4.7}$ & & 400 & $13.0_{-0.4}^{+0.2}$ \\
\hline 936 & $1_{-11.3}^{+15.8}$ & $29.5_{-5.2}^{+7.1}$ & $41.5_{-9.0}^{+12.2}$ & $28.8_{-9.5}^{+9.7}$ & $0.06_{-0.24}^{+0.24}$ & & & $67.0_{-10.8}^{+14.6}$ & & 3400 & \\
\hline & & & & & & & & & & & \\
\hline 190014 & & $6.09_{-0.06}^{+0.06}$ & & & $0.00_{-0}^{+0}$ & & & & & 19 & \\
\hline AW100828 & $58.0_{-4.8}^{+7.7}$ & & & & & & & & & 520 & $16.2_{-0.3}^{+0.2}$ \\
\hline 509 & & & & & & & & & & 660 & \\
\hline 149 & $75.0_{-17.6}^{+55.9}$ & $30.9_{-7.5}^{+17.2}$ & $45.8_{-13.3}^{+52.7}$ & $28.3_{-12.7}^{+13.4}$ & $-0.06_{-0.36}^{+0.37}$ & & & $72.0_{-16.8}^{+54.9}$ & & 4700 & \\
\hline & & & & & & & & & & 11000 & \\
\hline & & & & & & & & & & 400 & \\
\hline GW190924_021846 & $13.9_{-1.0}^{+5.1}$ & & & $5.0_{-1.9}^{+1.4}$ & & & $0.12_{-0.04}^{+0.04}$ & $13.3_{-1.0}^{+5.2}$ & & 360 & $11.5_{-0.4}^{+0.3}$ \\
\hline GW100020 012140 & $104.3_{-25.2}^{+34.9}$ & $35.8_{-8.2}^{+14.9}$ & $80.8_{-33.2}^{+33.0}$ & $24.1_{-10.6}^{+19.3}$ & $0.01_{-0.33}^{+0.34}$ & $2.13_{-1.05}^{+3.65}$ & $0.38_{-0.17}^{+0.49}$ & $101.5_{-25.3}^{+33.6}$ & & 2200 & $10.1_{-0.8}^{+0.6}$ \\
\hline 90930_133541 & & & $12.3_{-2.3}^{+12.4}$ & & & $0.76_{-0.32}^{+0.36}$ & & & & 1700 & \\
\hline
\end{tabular}




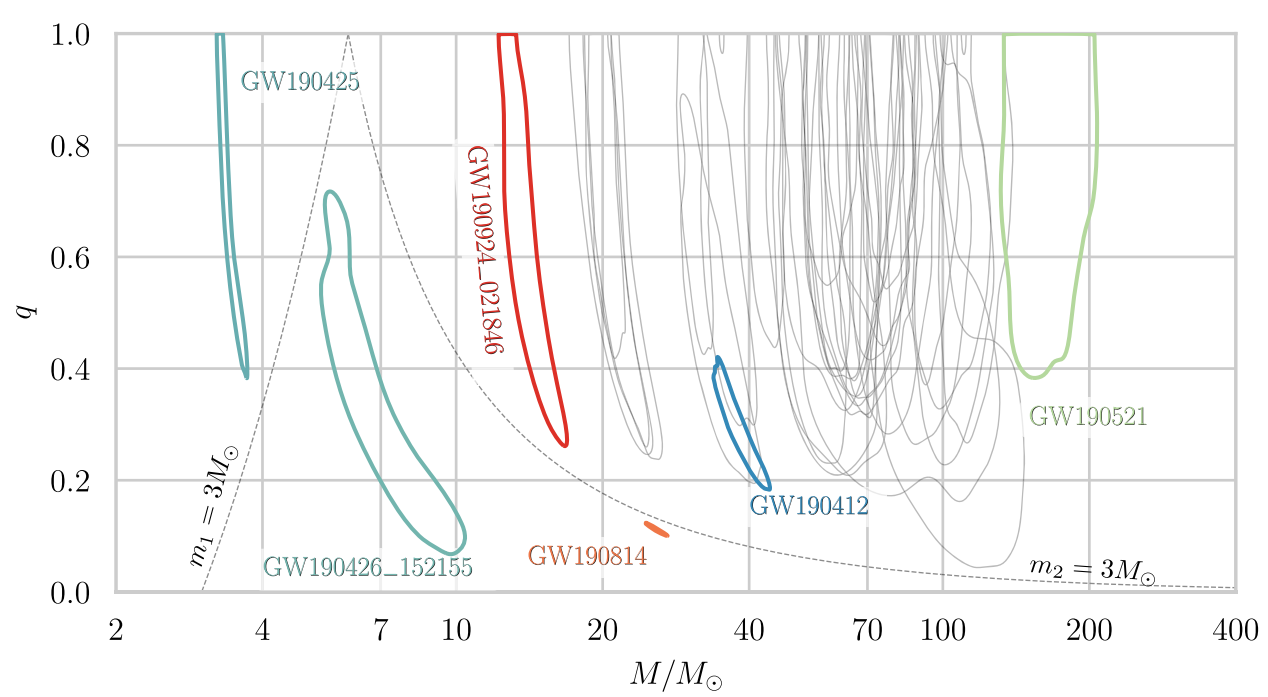

FIG. 6. Credible region contours for all candidate events in the plane of total mass $M$ and mass ratio $q$. Each contour represents the 90\% credible region for a different event. We highlight the previously published candidate events: GW190412, GW190425, GW190521, and GW190814, the potential NSBH GW190426_152155, and, finally, GW190924_021846, which is most probably the least massive system with both masses $>3 M_{\odot}$. The dashed lines delineate regions where the primary or secondary can have a mass below $3 M_{\odot}$. For the region above the $m_{2}=3 M_{\odot}$ line, both objects in the binary have masses above $3 M_{\odot}$.

$\left(M=13.9_{-1.0}^{+5.1} M_{\odot}\right)$ and likely also has the least massive object over $3 M_{\odot}\left(85 \%\right.$ probability and $\left.m_{2}=5.0_{-1.9}^{+1.4} M_{\odot}\right)$.

For most sources detected in $\mathrm{O} 3 \mathrm{a}$, the mass ratio posteriors have support at unity and, therefore, are consistent with equal mass mergers. An exception is the source of GW190412, which is the first event detected that has a confidently unequal mass ratio $\left(q=0.28_{-0.06}^{+0.12}\right)$ and exhibits strong signs of HM contributions to the waveform [236]. Although its mass ratio is confidently bounded away from unity, GW190412 has only a 34\% chance of having the smallest mass ratio among O3a sources with $m_{2}>3 M_{\odot}$.
As seen in Figs. 6 and 8, the mass ratios are not well constrained for many systems, so one or more could have a smaller mass ratio than GW190412.

\section{B. Sources with $m_{2}<3 M_{\odot}$ \\ 1. GW190425}

The least massive $\mathrm{O} 3 \mathrm{a}$ system is associated with GW190425 and is likely a binary neutron star system given the inferred masses $\left(m_{1}=2.0_{-0.3}^{+0.6} M_{\odot}\right.$ and $m_{2}=$ $1.4_{-0.3}^{+0.3} M_{\odot}$ ), but constraints on the tidal parameters do

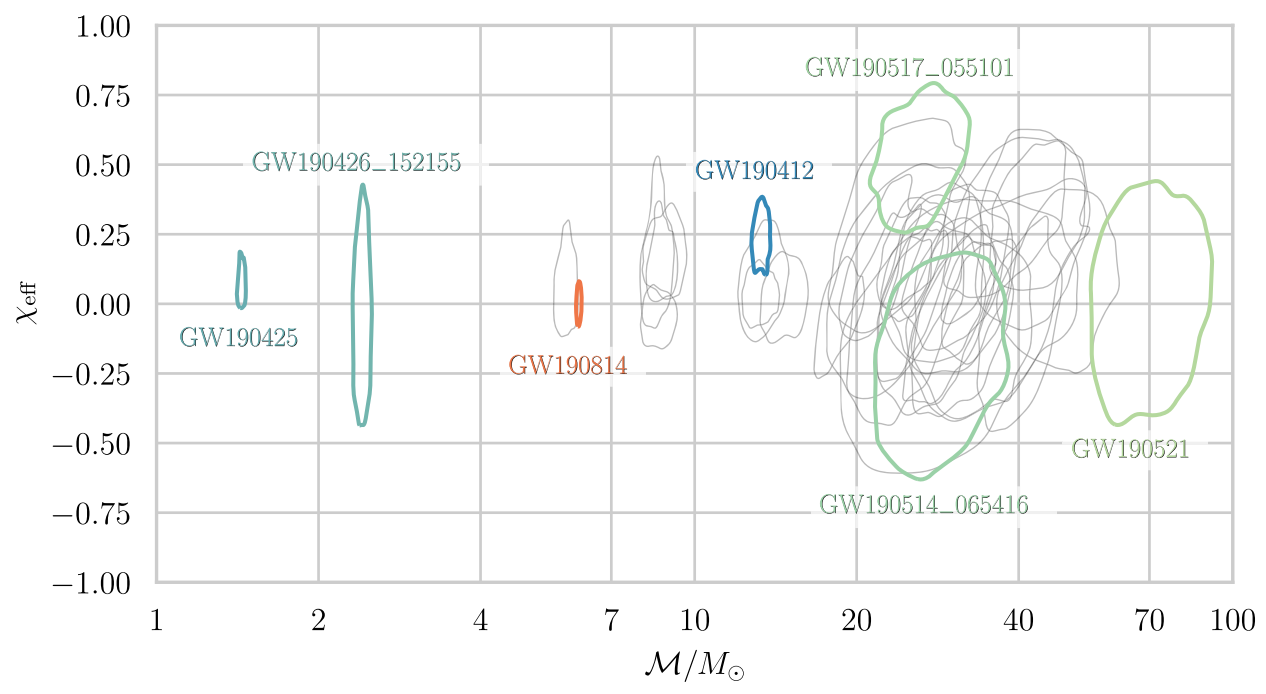

FIG. 7. Credible region contours for all candidate events in the plane of chirp mass $\mathcal{M}$ and effective inspiral spin $\chi$ eff . Each contour represents the $90 \%$ credible region for a different event. We highlight the previously published candidate events (cf. Fig. 6), as well as GW190517_055101 and GW190514_065416, which have the highest probabilities of having the largest and smallest $\chi$ eff , respectively. 


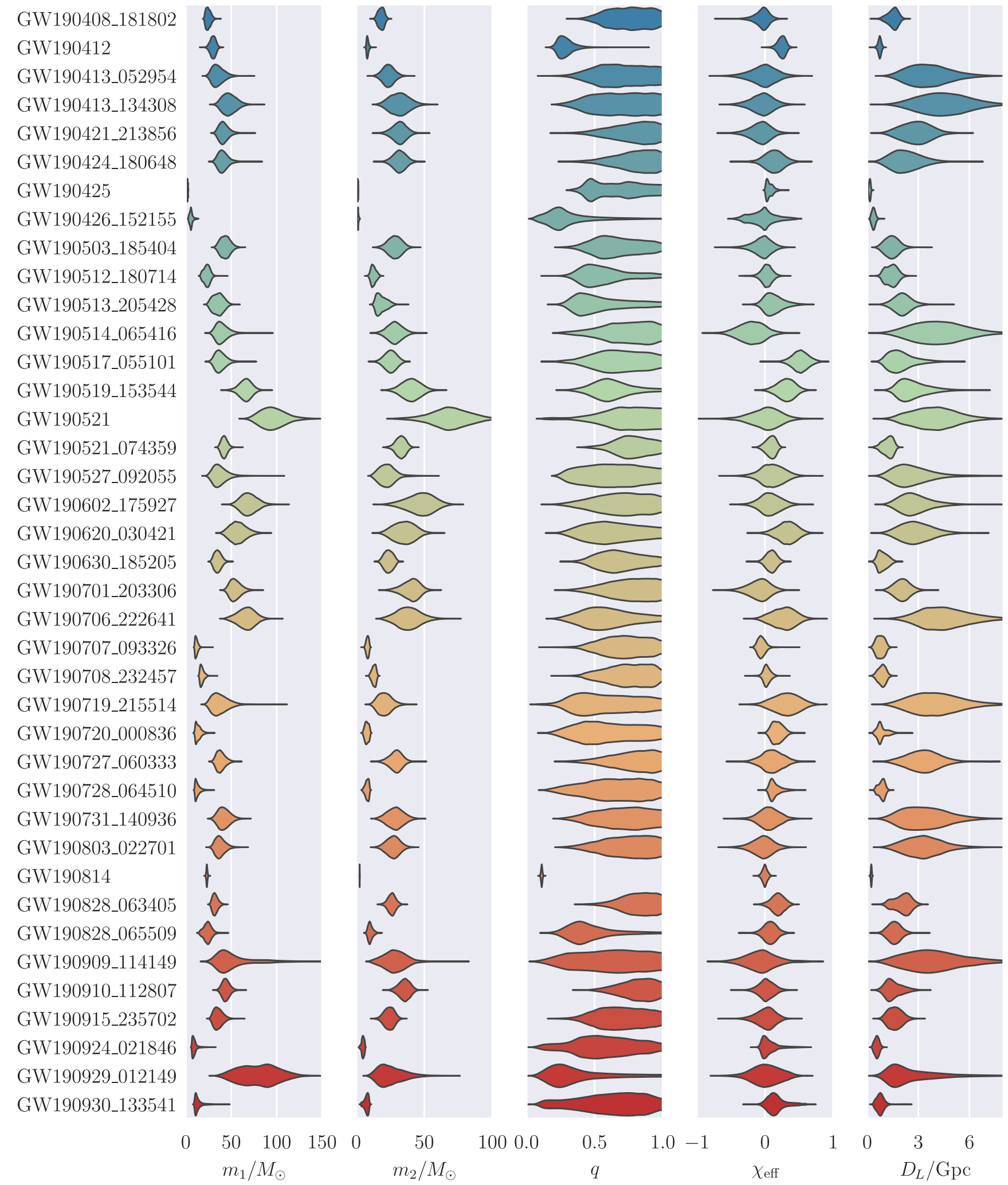

FIG. 8. Marginal posterior distributions on primary mass $m_{1}$, secondary mass $m_{2}$, mass ratio $q$, effective inspiral spin $\chi$ eff, and the luminosity distance $d_{L}$ for all candidate events in $\mathrm{O} 3 \mathrm{a}$. The vertical extent of each colored region is proportional to one-dimensional marginal posterior distribution at a given parameter value for the corresponding event. 
not rule out a NSBH or BBH origin. These estimates are obtained with the PhenomPv2NRT model with a high-spin prior that restricts dimensionless spin magnitudes of the compact objects to be less than 0.89 and previously reported in Ref. [32].

Although the inferred component masses of GW190425's source are consistent with masses of known neutron stars [270-273], the total mass $3.4_{-0.1}^{+0.3} M_{\odot}$ is greater than that of observed Galactic BNSs [274,275]. This difference raises the question of whether GW190425's source was formed in a different environment from the double neutron star systems observed to date [32,276-279].

Extending the discussion of waveform systematics reported with the discovery of GW190425 [32], we perform four supplementary analyses using the nonprecessing EOB models SEOBNRv4T_surrogate and TEOBResums for low-spin and high-spin priors. The results are summarized in Table VII. All the analyses produce quantitatively similar results to the corresponding nonprecessing analysis reported in the discovery paper, and the two EOB models produce consistent results between them.

For the low-spin prior, the results agree with the nonprecessing IMRPhenomDNRTidal analysis reported in the discovery paper, with SEOBNRv4T_surrogate and TEOBResumS recovering chirp mass as $\mathcal{M}=1.44_{-0.02}^{+0.02} M_{\odot}$ and effective inspiral spin of $\chi_{\text {eff }}=0.01_{-0.01}^{+0.01}$. When allowing larger compact object spins, the results with IMRPhenomDNRTidal and EOB models exhibit some differences but, overall, give consistent posteriors.

Our inferences about tidal parameters are likewise consistent with the previous nonprecessing analyses. We follow the procedure of Ref. [32] and reweight the posteriors to a flat in $\tilde{\Lambda}$ prior. For the low-spin prior, the two EOB models give very similar bounds, with $\tilde{\Lambda}$ constrained

TABLE VII. Source properties of GW190425 with different waveform families. For the primary mass we give the $0 \%-90 \%$ interval, while for the secondary mass and mass ratio we give the $10 \%-100 \%$ confidence intervals. The quoted $90 \%$ upper limits for $\tilde{\Lambda}$ are obtained by reweighing its posterior distribution as detailed in Appendix F of Ref. [32]. The top half of the table describes values from low-spin prior (LS), while bottom half for high-spin prior (HS). For LS, all the results are consistent with each other, while for HS there are slight differences among different waveforms.

\begin{tabular}{lcccc}
\hline \hline Waveform & $m_{1} / M_{\odot}$ & $m_{2} / M_{\odot}$ & $q$ & $\tilde{\Lambda}$ \\
\hline IMRPhenomD_NRTidal & $1.6-1.9$ & $1.5-1.7$ & $0.77-1.0$ & $\leq 580$ \\
IMRPhenomPv2_NRTidal & $1.6-1.9$ & $1.5-1.7$ & $0.77-1.0$ & $\leq 580$ \\
SEOBNRv4T_surrogate & $1.6-1.9$ & $1.5-1.7$ & $0.77-1.0$ & $\leq 630$ \\
TEOBResums & $1.6-1.9$ & $1.5-1.7$ & $0.77-1.0$ & $\leq 600$ \\
\hline IMRPhenomD_NRTidal & $1.6-2.5$ & $1.1-1.7$ & $0.46-1.0$ & $\leq 1090$ \\
IMRPhenomPv2_NRTidal & $1.6-2.5$ & $1.1-1.7$ & $0.45-1.0$ & $\leq 1080$ \\
SEOBNRv4T_surrogate & $1.6-2.3$ & $1.6-2.3$ & $0.54-1.0$ & $\leq 1040$ \\
TEOBResumS & $1.6-2.3$ & $1.2-1.7$ & $0.54-1.0$ & $\leq 870$ \\
\hline \hline
\end{tabular}
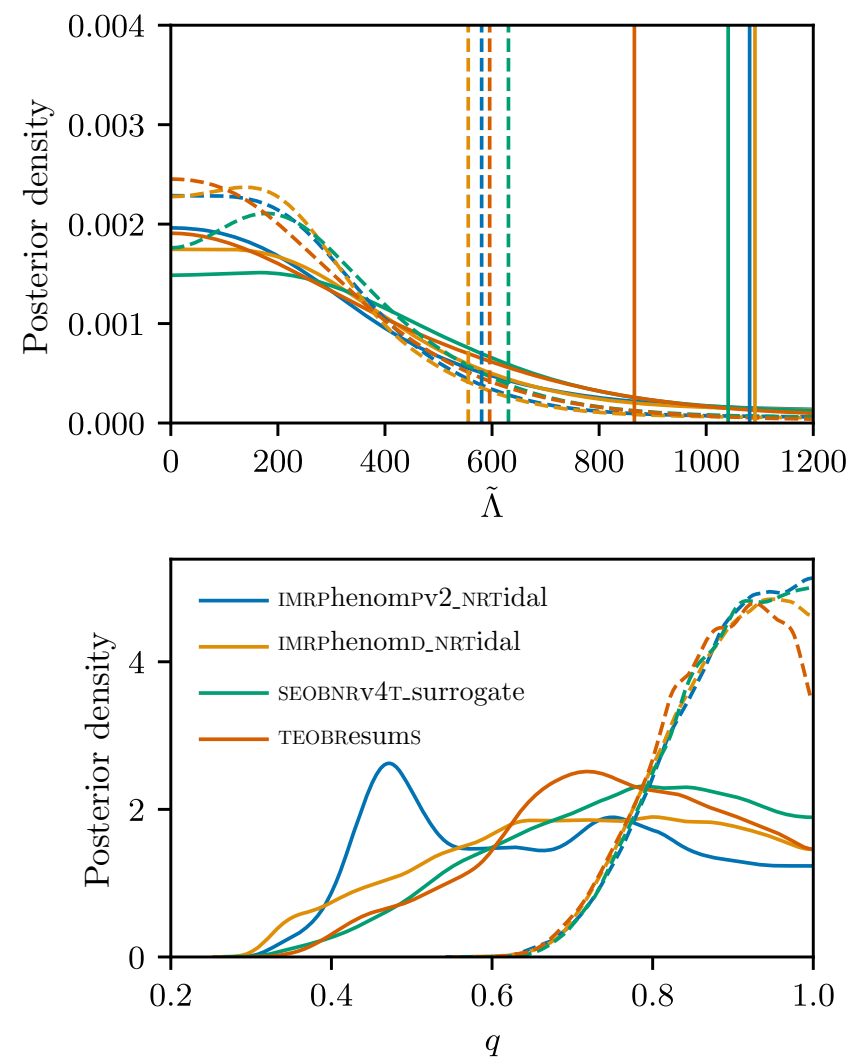

FIG. 9. Marginalized distributions for the combined dimensionless tidal deformability parameter $\tilde{\Lambda}$ (top) and for mass ratio (bottom) for BNS event GW190425. The solid lines correspond to the high-spin prior, while the dashed to the low-spin prior. In the top panel, the vertical lines mark the corresponding $90 \%$ upper bound on $\tilde{\Lambda}$. SEOBNRv4T_surrogate constrains the mass ratio better toward equal masses for high larger spins. The plot and quoted $90 \%$ upper limits for $\tilde{\Lambda}$ are obtained by reweighing posterior distribution as described in Appendix F of Ref. [32].

below 630. For the high-spin prior, the TEOBResums waveform model constrains the dimensionless tidal deformability parameter better $(\tilde{\Lambda} \leq 870)$ as compared to the other waveform models, as seen in the top in Fig. 9.

The two EOB models also constrain the mass ratio better than other waveforms $(0.54-1.0)$ as can be seen in the bottom in Fig. 9. The previously reported analyses that allow for significant precessing spins have much greater flexibility and, thus, for the high-spin prior, produce a more asymmetric mass ratio posterior distribution than the two nonprecessing updates reported here.

Finally, both the EOB models find the luminosity distance of $D_{L}=0.16_{-0.07}^{+0.07} \mathrm{Gpc}$ independent of the spin prior used.

\section{GW190814}

Among the O3a events, GW190814's source [33] has the least massive secondary component after the sources of GW190425 and GW190426_152155. GW190814's less 
massive component has mass $m_{2}=2.59_{-0.09}^{+0.08} M_{\odot}$, making its interpretation as a black hole or a neutron star unclear [33]. GW190814 also has the most extreme mass ratio of all the candidate events, $q=0.112_{-0.009}^{+0.008}$ [33].

\section{GW190426 152155}

GW190426_152155 is the candidate event with the highest FAR: $1.4 \mathrm{yr}^{-1}$. Assuming it is a real signal of astrophysical origin, we estimate its component masses to be $m_{1}=5.7_{-2.3}^{+3.9} M_{\odot}$ and $m_{2}=1.5_{-0.5}^{+0.8} M_{\odot}$, raising the possibility that it could have originated from either a $\mathrm{BBH}$ or an NSBH source. The mass of the secondary component is consistent with masses of (previously) reported neutron stars [10,271,272,274], but the data are uninformative about potential tidal effects, showing essentially no difference between the prior and posterior on $\Lambda_{2}$ obtained from NSBH waveforms SEOBNRv4_ROM_ NRTidalv2_NSBH or IMRPhenomNSBH which we use for our fiducial results. A more definitive assessment of this event likely requires further observations to establish the rate of astrophysical signals with comparable properties.

\section{Spins}

Most of the compact objects detected in O3a have spin magnitudes consistent with zero, within uncertainties, but in some cases the spins can be constrained away from zero. Two systems have a $>50 \%$ chance of having at least one black hole with dimensionless spin magnitude $\chi_{i=\{1,2\}}>$ 0.8: GW190517_055101 has $\chi_{i=\{1,2\}}>0.8$ with $77 \%$ credibility, and GW190521 with 58\% credibility. In addition to spin magnitudes, we also consider the effective inspiral spins. As described in Sec. IV B, the effective inspiral spin $\chi_{\text {eff }}$ is the mass-weighted combination of aligned spins and is approximately conserved under precession. Effective inspiral spin posterior distributions for all candidate events are shown in Figs. 7 and 8. We find 11 systems that show signs of nonzero $\chi_{\text {eff }}$. At $90 \%$ credibility, GW190412, GW190425, GW190517_055101, GW190519_153544, GW190620_030421, GW190706_ 222641, GW190719_215514, GW190720_000836, GW190728_064510, GW190828_063405, and GW190930_133541 have sources with $\chi_{\text {eff }}>0$. No individual systems are found to have $\chi_{\text {eff }}<0$ with $\geq 95 \%$ probability, but the event with the lowest $\chi_{\text {eff }}$ in O3a is probably GW190514_065416 with $\chi_{\text {eff }}=-0.19_{-0.32}^{+0.29}$. The surplus of events with $\chi_{\text {eff }}>0$ compared to none with $\chi_{\text {eff }}<0$ suggests that the spin orientations of black holes in binaries are not isotropically distributed with respect to their orbital angular momenta. This possibility is explored further in Ref. [37].

The BBH which most likely has the largest measured $\chi_{\text {eff }}$ is GW190517_055101 $\left(\chi_{\text {eff }}=0.52_{-0.19}^{+0.19}\right)$. It has a $60 \%$ posterior probability of having the highest $\chi_{\text {eff }}$, followed by GW190719_215514 $\left(\chi_{\text {eff }}=0.32_{-0.31}^{+0.29}\right)$ with $12 \%$.
In some cases, the joint $\chi_{\text {eff }}$ and mass-ratio measurement for an event enables a tighter measurement of the spin magnitude of the primary mass than for candidate events with mass ratios closer to unity. For example, we find primary spin magnitudes of $\chi_{1}=0.40_{-0.35}^{+0.40}$ for the source of GW190720_000836 and $\chi_{1}=0.32_{-0.28}^{+0.37}$ for the source of GW190728_064510. Posterior distributions on spin magnitudes and tilt angles are shown in Fig. 10 for these two candidate events and other select systems that exhibit nonzero spins.

The magnitude of spin precession in the waveform can be partially captured by the effective precession spin parameter $\chi_{p}$, which includes the projection of component spin vectors onto the orbital plane [197,198]. To identify events for which the data constrain $\chi_{p}$, we compare the $\chi_{p}$ priors - conditioned on the posteriors of $\chi_{\text {eff }}$ - to the $\chi_{p}$ posteriors, as done in Ref. [8]. Conditioning the $\chi_{p}$ prior on the $\chi_{\text {eff }}$ posterior accounts for the correlated prior between $\chi_{\text {eff }}$ and $\chi_{p}$ in the default spin prior choices presented in Sec. V. Figure 11 shows the one-dimensional posterior and ( $\chi_{\text {eff-conditioned) }}$ prior distributions on $\chi_{p}$ for events with Jensen-Shannon (JS) divergence [280] $D_{\mathrm{JS}}^{\chi_{p}}>0.05$ bit, where $D_{\mathrm{JS}}^{\chi_{p}}$ is calculated between the $\chi_{p}$ posterior and conditioned prior. For most of the candidate events, the posterior on $\chi_{p}$ is similar to the prior, indicating that the data are largely uninformative about precession, but there are a few notable exceptions. The $\chi_{p}$ inference on GW190814 is most striking with $D_{\mathrm{JS}}^{\chi_{p}}=0.72$ bit: The spin magnitude of the primary mass of the system is constrained to be near zero, resulting in a correspondingly small $\chi_{p}$ value. After GW190814, $D_{\text {JS }}^{\chi_{p}}$ is largest for GW190412 [236,281] and GW190521 [34,35], with $D_{\text {JS }}^{\chi_{p}}=0.18$ bit and $D_{\mathrm{JS}}^{\chi_{p}}=0.15$ bit, respectively. Unlike GW190814, the $\chi_{p}$ posterior distributions for these events are constrained away from zero, showing preference for precession in these systems. The tilt angle of GW190412's more massive component's spin with respect to the Newtonian orbital angular momentum is particularly well constrained to $\theta_{L S_{1}}=0.80_{-0.35}^{+0.54}$, as seen in Fig. 10. To further investigate possible precession in these signals, we compute the precession SNR $\rho_{p}$ [282,283], which characterizes the observability of precession in gravitational-wave data. GW190412 has the largest precession SNR in O3a with a median $\rho_{p}=3.0$. Despite parameter estimation preferring high $\chi_{p}$ for GW190521, we calculate $\rho_{p}=0.7$ showing that the measurable precession in the signal is small due to the short signal duration. Using empirical relations between $\rho_{p}$ and Bayes' factors for precession [284,285], we see that the corresponding Bayes' factors for precession also only mildly favor precession. A population-level analysis of the GWTC-2 spins is presented in Ref. [37] and finds evidence for the presence of spin precession in the population. 


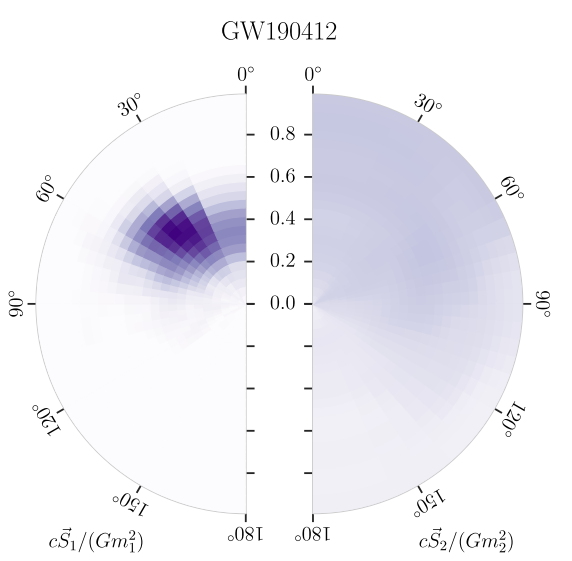

GW190620_030421

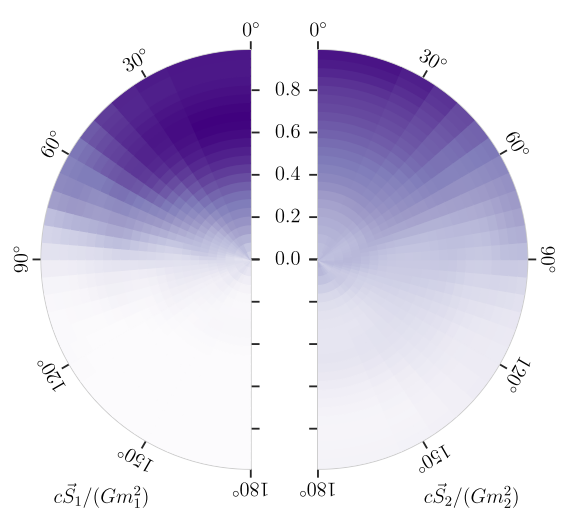

GW190517_055101

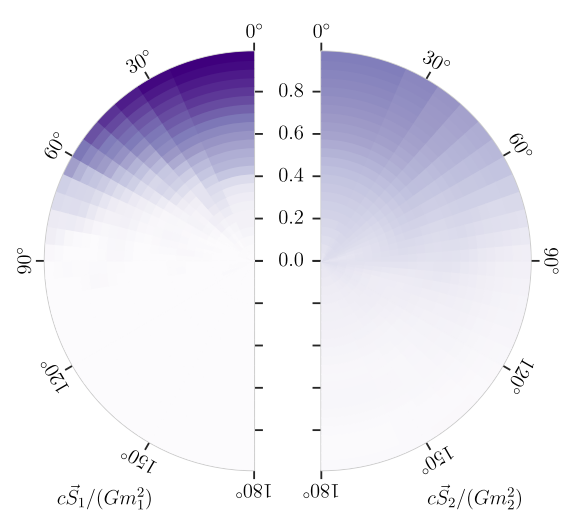

GW190720_000836

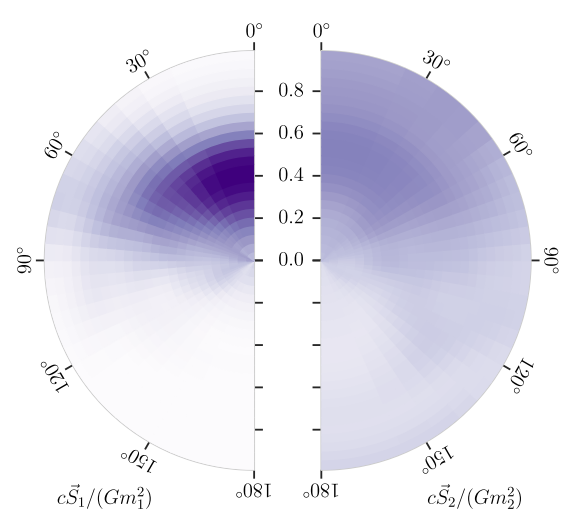

GW190519_153544

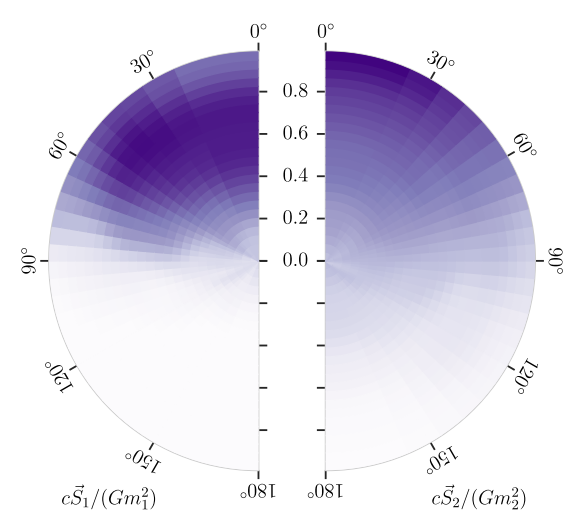

GW190728_064510

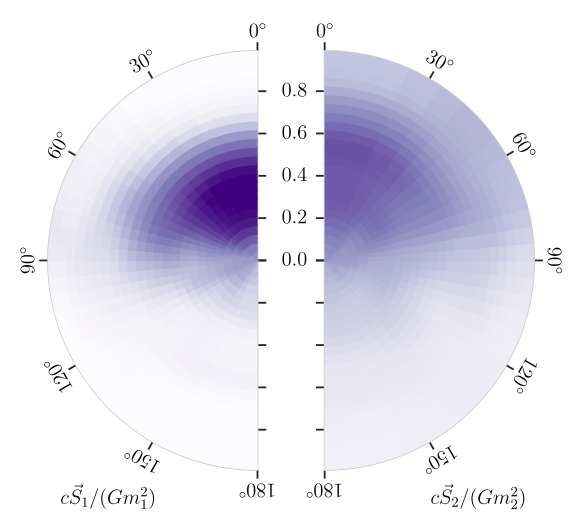

FIG. 10. Estimates of the dimensionless spin parameters $\vec{\chi}_{i}=c \vec{S}_{i} /\left(G m_{i}^{2}\right)$ for merger components of selected sources. Each pixel's radial distance from the circle's center on the left (right) side of each disk corresponds to the spin magnitude $|\vec{\chi}|$ of the more (less) massive component, and the pixel's angle from the vertical axis indicates the tilt angle $\theta_{\mathrm{LS}}$ between each spin and the Newtonian orbital angular momentum. Pixels have equal prior probability, and shading denotes the posterior probability of each pixel, after marginalizing over azimuthal angles.

\section{Three-dimensional localization}

The most distant event, after accounting for measurement uncertainties in distance, is most probably GW190413_134308, with an estimated luminosity distance and redshift of $D_{L}=4.45_{-2.12}^{+2.48} \mathrm{Gpc}$ and $z=0.71_{-0.30}^{+0.31}$, respectively, approximately twice the luminosity distance of the most distant source from GWTC-1, GW170729 [8], and comparable to the distance for gravitational-wave candidate GW170817A [22] (not to be confused with the BNS signal GW170817). However, GW190909_ 114149, GW190514_065416, GW190521, GW190706_ 222641, and GW190719_215514 have similarly large distances to GW190413_134308. With candidate events at these cosmological distances, we can more readily measure the Hubble constant and the evolution of the $\mathrm{BBH}$ merger rate over cosmic time. Such analyses are performed in Ref. [37]. The closest source detected in O3a is GW190425, with an inferred luminosity distance of $D_{L}=0.16_{-0.07}^{+0.07} \mathrm{Gpc}$, about 4 times the distance for GW170817.

Overall, GW190814 is the best localized event detected in O3a. The contour encompassing $90 \%$ of this event's two-dimensional sky position posterior is $\Delta \Omega=19 \mathrm{deg}^{2}$, and $90 \%$ of this event's $3 \mathrm{D}$ sky position posterior is contained in $\Delta V_{90}=3.2 \times 10^{-5} \mathrm{Gpc}^{3}$. Although GW190814 was initially detected in only LIGO Livingston and Virgo, it was reanalyzed with LIGO Hanford data, enabling the strong constraint on 3D source position. GW190412 and GW190701_203306 are also relatively well localized with $\Delta \Omega=21 \mathrm{deg}^{2}, \Delta V_{90}=0.035 \mathrm{Gpc}^{3}$ and $\Delta \Omega=46 \mathrm{deg}^{2}$, $\Delta V_{90}=0.035 \mathrm{Gpc}^{3}$, respectively, and are both detected in all three detectors. GW190424_180648 is detected and analyzed only in Livingston data and, therefore, has the largest localization area and volume with $\Delta \Omega=28000 \mathrm{deg}^{2}$ and $\Delta V_{90}=28 \mathrm{Gpc}^{3}$. Credible intervals on each source's distance and sky area are shown in Table VI. Probability density sky maps for all events are available as part of the data release [262].

\section{WAVEFORM RECONSTRUCTIONS}

Template-based $[179,286]$ and minimally modeled methods $[107,112,287]$ are complementary techniques for producing waveform reconstructions. Waveform templates 


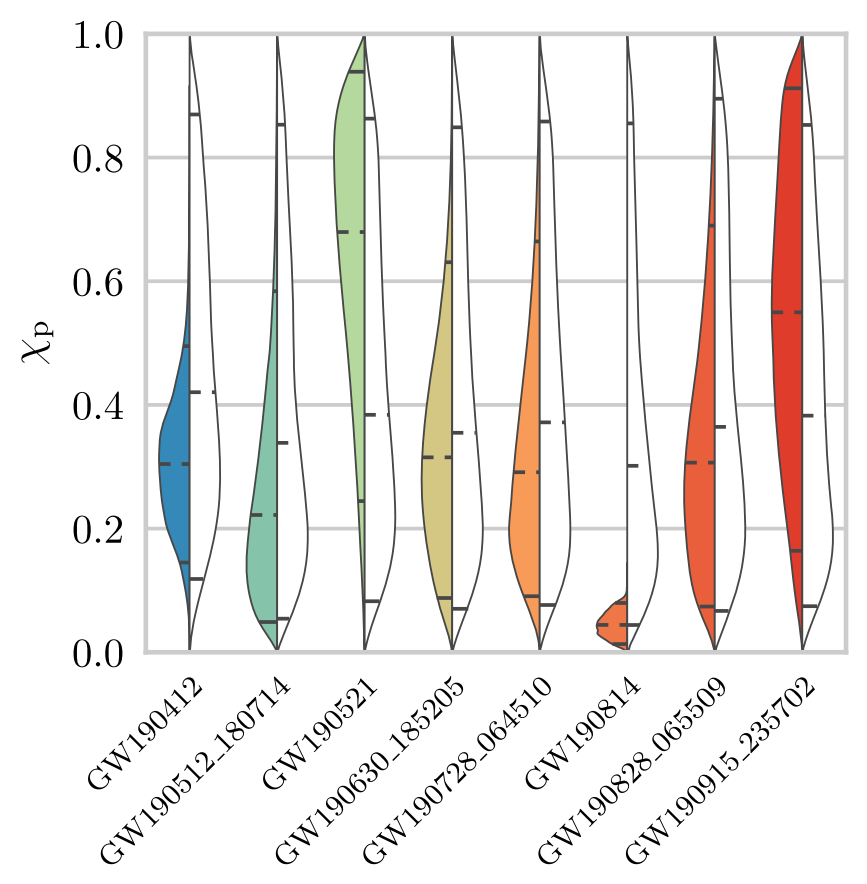

FIG. 11. Posterior and prior distributions on the effective precession spin parameter $\chi_{p}$ for select events. The $\chi_{p}$ prior shown on the right half of each leaf is conditioned on the posterior of $\chi_{\text {eff }}$, since the default prior in sampling contains correlations between $\chi_{p}$ and $\chi_{\text {eff }}$.

provide a mapping between the shape of the waveforms and the parameters of the source, such as the masses and spins of a binary system, but are limited to those sources for which we have models. Minimally modeled reconstructions make it possible to discover unexpected phenomena, but they do not provide a direct mapping to the physical properties of the source. Currently available waveform templates for binary mergers are based on various approximations and numerical solutions to Einstein's equations that cover a subset of the full parameter space. These waveform templates may, thus, fail to capture some features of the signal. A more exotic possibility is that gravity behaves differently than predicted by general relativity. One way to test these scenarios is to compare the template-based and minimally modeled waveform reconstructions.

A standard measure of the agreement between two waveforms $h_{1}$ and $h_{2}$ is the match, or overlap

$$
\mathcal{O}\left\langle h_{1}, h_{2}\right\rangle=\frac{\left\langle h_{1} \mid h_{2}\right\rangle}{\sqrt{\left\langle h_{1} \mid h_{1}\right\rangle\left\langle h_{2} \mid h_{2}\right\rangle}},
$$

where $\langle a \mid b\rangle$ denotes the noise-weighted inner product $[143,180]$. The match is constrained to be $\leq 1$.

For each event, the matches are computed between the maximum likelihood template-based waveforms and two minimally modeled waveform reconstruction methods, cWB [112] and BW [107], using data that contain the
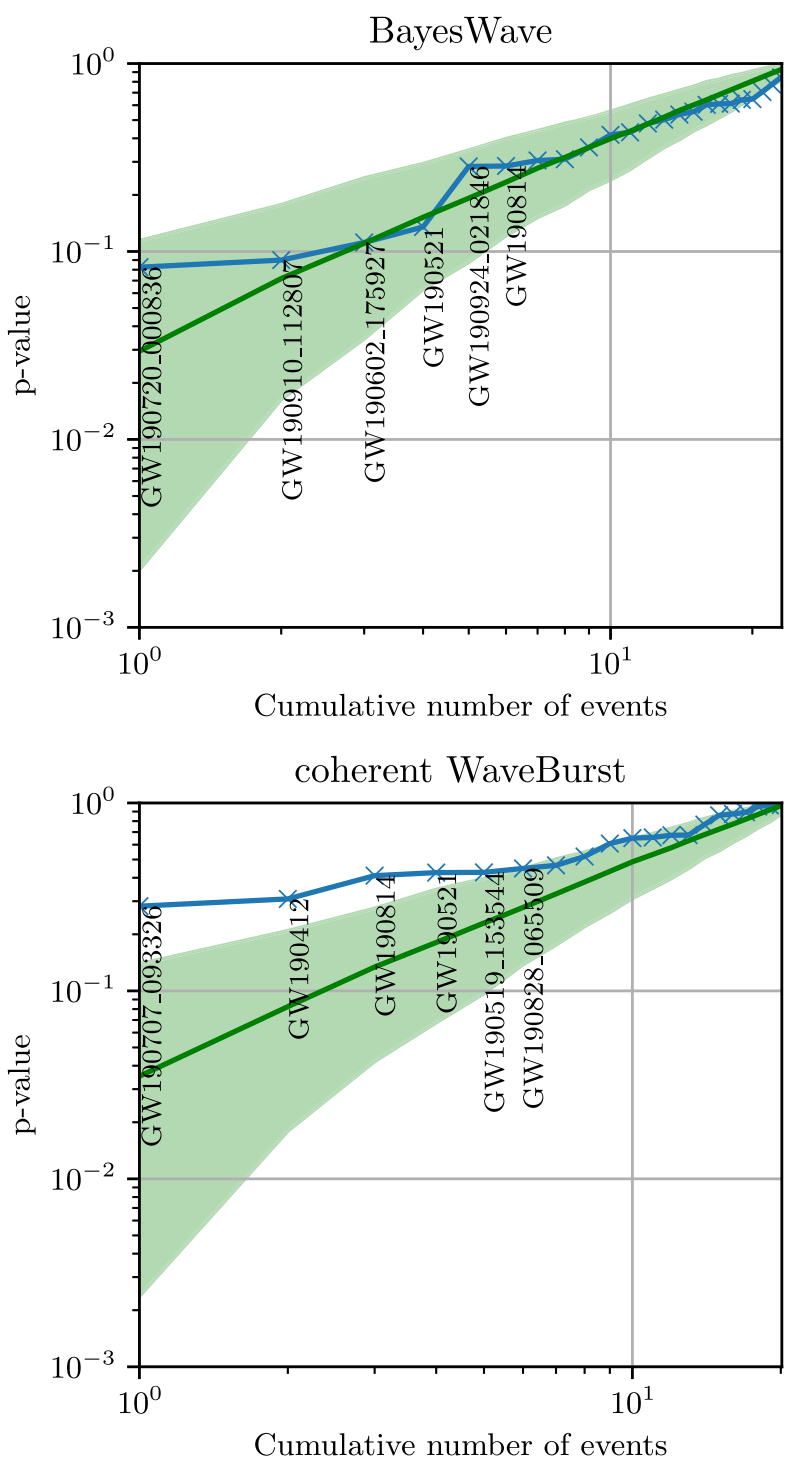

FIG. 12. $p$-value plot for the candidate events reconstructed by the minimally modeled pipelines in O3a. The upper panel is from the BW analysis, and the lower panel is from the cWB analysis. The cWB analysis includes the candidate events that are both detected and reconstructed (listed in Table IV) and those that are detected but reconstructed offline. (GW190513_205428, GW190707_093326, GW190728_064510, GW190814, and GW190828_065509). The BW analysis uses the same selection of $\mathrm{BBH}$ candidate events as are used for testing general relativity [36]. The $p$ values are sorted in increasing order and plotted vs the order number (which is also the cumulative number of candidate events). Each $p$ value is obtained from the observed on-source match value and the corresponding off-source distribution of the match values from off-source injections. The green band indicates the theoretical $90 \%$ symmetric distribution about the null hypothesis (dark green line). Only deviations below the green $90 \%$ confidence band indicate disagreement, and we see that there are none. A few of the cWB $p$ values are above the band, indicating either a statistical fluctuation or an overfitting, which we attribute to a small asymmetry between the way the on-source and off-source matches are computed. 
event (on-source data). To ascertain whether these match values are in line with expectations, waveforms from the template-based analysis are added to data near, but not including, each event (off-source data). The minimally modeled waveform reconstructions are repeated multiple times on these off-source data to estimate the distribution of match values we would expect for each event. For each event, these distributions are used to compute a $p$ value, given by the fraction of off-source match values that are below the on-source match. For some of the lower SNR candidate events, the minimally modeled methods are unable to reconstruct the signals, and these candidate events are excluded from the analysis. Details of the analysis procedure, and additional results, can be found in Appendix B.

Figure 12 shows the $p$ values for the analyzed candidate events sorted in increasing order [287,288]. Events with $p$ values above the diagonal have on-source matches that are higher than expected, while those below the line have matches that are lower than expected. The cWB analysis shows some events outside the theoretical $90 \%$ band for the single-order statistic, which can be due to statistical fluctuations. Because of correlations between $p$ values in the ordered plot, for $20 p$ values the probability of finding exactly four $p$ values outside the $90 \%$ band as in Fig. 12 is $5.2 \%$, although it may also point to a systematic effect in the analysis. Since the $p$ values are higher than expected, there is no evidence of a discrepancy with the templatebased analysis. Our tests also indicate that, if there is indeed a systematic effect, it may originate from an overestimate of the off-source matches no larger than $2 \%$. Further details are given in Appendix B. Overall, the $p$-value distributions support the null hypothesis that the minimally modeled waveform reconstructions are consistent with the generalrelativity-derived waveform templates.

\section{CONCLUSION}

We have presented the results from a search for compact binary coalescence signals in the first part of the third observing run of Advanced LIGO and Advanced Virgo. During the period of observations, spanning 1 April to 1 October 2019, the three detectors had sensitivity that significantly exceeded previous observing runs, with median BNS inspiral ranges of 108 (Hanford), 135 (Livingston), and $45 \mathrm{Mpc}$ (Virgo). This improved sensitivity allowed us to greatly expand the number of known compact binary mergers, adding 39 new gravitational-wave events to the 11 we have previously reported in GWTC-1 [8].

We performed parameter estimation on these 39 new GWTC-2 signals using a range of waveform models, allowing us to incorporate the effect of HMs in the inference of source parameters for $\mathrm{BBH}$ systems and to compare to the systematic differences between waveform families. We find that the sources of these signals include $\mathrm{BBHs}$ that are more massive, farther away, and more asymmetric in mass ratio than any sources in GWTC-1, as well as three binaries with at least one component of mass $<3 M_{\odot}$. These latter systems may include the first detected NSBH mergers; however, there is insufficient SNR to perform an informative measurement of the tidal deformability that would definitively indicate whether they had an NSBH or BBH origin. We expanded our analysis of the potential BNS signal to encompass further waveforms which include tidal effects, finding agreement with previously reported results [32].

Our analyses recovered 11 sources with positive effective inspiral spin but zero with negative (at $90 \%$ credibility). Although no individual binary in O3a has $\chi_{\text {eff }}<0$ with high credibility, a hierarchical population analysis [37] of LVC-reported events to date indicates a nontrivial fraction of binaries have negative effective inspiral spin. This result would be consistent with the independent observation of gravitational-wave candidate GW170121, which was found to have significant probability of having $\chi_{\text {eff }}<0$ [23,27]. We also examined the evidence for misalignment of orbital and component angular momenta, which would be an indication of a dynamical formation channel and produce precession of the orbital plane. We find only mild evidence in favor of precession in the most significant case of GW190412 [236,281]. We also performed consistency checks between the waveform models and the observed data, finding no statistically significant differences.

A pair of companion papers make use of the events in GWTC-2 to study source populations and fundamental physics. The inferred population distribution of compact object mergers is described in Ref. [37], which reports an updated BBH merger rate density of 23.9 $9_{-8.6}^{+14.9} \mathrm{Gpc}^{-3} \mathrm{yr}^{-1}$ and rate density for BNS of $320_{-240}^{+490} \mathrm{Gpc}^{-3} \mathrm{yr}^{-1}$. This paper also investigates mass and spin distributions and finds evidence for a population of BBH systems with spins misaligned from the orbital plane. Eight tests of general relativity are reported in Ref. [36], showing no evidence for violations of Einstein's theory of gravity, leading to some of the best constraints on alternative theories to date.

The online gravitational-wave transient catalog represents a cumulative set of events found in LIGO-Virgo data, including detections presented in GWTC-1 and now also events from the first six months of the $\mathrm{O} 3$ observing run. O3 continued from November 2019 until March 2020. Analysis of the second portion (O3b) is currently in progress, and events found during this period will be added in the next update.

The Advanced LIGO and Advanced Virgo detectors are currently undergoing commissioning to further improve their sensitivity and will be joined in their fourth observing run by the KAGRA detector [289]. This upgrade should lead to improvements in the detection rate and source localization, improving the prospects for multimessenger observation of future sources $[290,291]$. 
Data products associated with this catalog are available through the Gravitational Wave Open Science Center (GWOSC) [262]. Data associated with all events described in this paper are available through the GWOSC event portal, including calibrated strain time series, parameter estimation posterior samples, tables of $90 \%$ credible intervals for physical parameters, and search pipeline results. These data products may be accessed through a Web browser or open-source client package [292]. We provide a public data release associated with the results contained in this paper [293], which includes the data behind the figures, the simulation data used in estimating search sensitivity, and the posterior samples used in estimating the source properties. Full results from the injection campaign are available via a data release [294].

\section{ACKNOWLEDGMENTS}

The authors gratefully acknowledge the support of the United States National Science Foundation (NSF) for the construction and operation of the LIGO Laboratory and Advanced LIGO as well as the Science and Technology Facilities Council (STFC) of the United Kingdom, the Max-Planck-Society (MPS), and the State of Niedersachsen/Germany for support of the construction of Advanced LIGO and construction and operation of the GEO600 detector. Additional support for Advanced LIGO was provided by the Australian Research Council. The authors gratefully acknowledge the Italian Istituto Nazionale di Fisica Nucleare (INFN), the French Centre National de la Recherche Scientifique (CNRS), and the Netherlands Organization for Scientific Research, for the construction and operation of the Virgo detector and the creation and support of the EGO consortium. The authors also gratefully acknowledge research support from these agencies as well as by the Council of Scientific and Industrial Research of India, the Department of Science and Technology, India, the Science and Engineering Research Board (SERB), India, the Ministry of Human Resource Development, India, the Spanish Agencia Estatal de Investigación, the Vicepresidència i Conselleria d'Innovació, Recerca i Turisme and the Conselleria d'Educació i Universitat del Govern de les Illes Balears, the Conselleria d'Innovació, Universitats, Ciència i Societat Digital de la Generalitat Valenciana and the CERCA Programme Generalitat de Catalunya, Spain, the National Science Centre of Poland and the Foundation for Polish Science (FNP), the Swiss National Science Foundation (SNSF), the Russian Foundation for Basic Research, the Russian Science Foundation, the European Commission, the European Regional Development Funds (ERDF), the Royal Society, the Scottish Funding Council, the Scottish Universities Physics Alliance, the Hungarian Scientific Research Fund (OTKA), the French Lyon Institute of Origins (LIO), the Belgian Fonds de la Recherche Scientifique
(FRS-FNRS), Actions de Recherche Concertées (ARC) and Fonds Wetenschappelijk Onderzoek-Vlaanderen (FWO), Belgium, the Paris Île-de-France Region, the National Research, Development and Innovation Office Hungary (NKFIH), the National Research Foundation of Korea, the Natural Science and Engineering Research Council Canada, Canadian Foundation for Innovation (CFI), the Brazilian Ministry of Science, Technology, Innovations, and Communications, the International Center for Theoretical Physics South American Institute for Fundamental Research (ICTP-SAIFR), the Research Grants Council of Hong Kong, the National Natural Science Foundation of China (NSFC), the Leverhulme Trust, the Research Corporation, the Ministry of Science and Technology (MOST), Taiwan, and the Kavli Foundation. The authors gratefully acknowledge the support of the NSF, STFC, INFN, and CNRS for provision of computational resources.

The detection of the signals and subsequent significance evaluations in this catalog were performed with the GstLAL based inspiral software pipeline $[111,122,123]$ built on the LALSuite software library [295] and with the PyСBC [29,124-127] and cWB [112,118-121] packages. Parameter estimation was performed with the LALInference [179] and LALSimulation libraries within LALSuite [295], the Bilby and Parallel Bilby libraries [232-234] using the dynesty nested sampling package [235], and the RIFT library [229-231]. PESummary was used to postprocess and collate parameter estimation results [237]. Estimates of the noise spectra and glitch models were obtained using BayesWave [107,108,182]. The cWB and BayesWave packages were also used to generate waveform reconstructions. Plots were prepared with MATPLOTLIB [296], SEABORN [297], and GWPY [298]. NumPy [299] and SciPy [300] were used in the preparation of the manuscript.

\section{APPENDIX A: WAVEFORM SYSTEMATICS}

The choice of waveform influences our inferences of source properties $[8,301,302]$. As such, we employ multiple waveform families in the inference of each event's source parameters. The full list of models represented in our publicly available results for each event is shown in Table VIII.

There are many tools designed to quantify difference between probability distributions, one of which is the Kullback-Leibler (KL) divergence [303]. It is defined by

$$
D_{\mathrm{KL}}(p \mid q)=\int p(\vartheta) \log _{2}\left(\frac{p(\vartheta)}{q(\vartheta)}\right) d \vartheta
$$

where $p(\vartheta)$ and $q(\vartheta)$ are two probability distributions. However, the KL divergence is not symmetric, $D_{\mathrm{KL}}(p \mid q) \neq$ $D_{\mathrm{KL}}(q \mid p)$, and furthermore $D_{\mathrm{KL}}$ can diverge if the probability distributions are disjoint, which makes it impractical 
TABLE VIII. Summary of the waveform models used for the analyses, available in the data release. Fiducial results used for the main presentation in Sec. VII are shown in bold. Where multiple bold waveforms are listed, equal numbers of posterior samples from runs with those waveforms are combined. For GW190425, HS and LS suffixes correspond to high-spin $\left(\left|\overrightarrow{\chi_{i}}\right| \leq 0.89\right)$ and low-spin $\left(\left|\vec{\chi}_{i}\right| \leq 0.05\right)$ priors, respectively.

\begin{tabular}{|c|c|}
\hline Event & Available runs \\
\hline GW190408_181802 & IMRPhenomD, IMRPhenomPv2, SEOBNRv4P, SEOBNRv4PHM \\
\hline GW190412 & $\begin{array}{c}\text { IMRPhenomD, IMRPhenomHM, IMRPhenomPv2, IMRPhenomPv3HM, SEOBNRv4HM_ROM, } \\
\text { SEOBNRv4P, SEOBNRv4PHM, SEOBNRv4_ROM }\end{array}$ \\
\hline GW190413_052954 & IMRPhenomD, IMRPhenomPv2, NRSur7dq4, SEOBNRv4P, SEOBNRv4PHM \\
\hline GW190413_134308 & IMRPhenomD, IMRPhenomPv2, NRSur7dq4, SEOBNRv4P \\
\hline GW190421_213856 & IMRPhenomD, IMRPhenomPv2, NRSur7dq4, SEOBNRv4P, SEOBNRv4PHM \\
\hline GW190424_180648 & IMRPhenomD, IMRPhenomPv2, NRSur7dq4, SEOBNRv4P, SEOBNRv4PHM \\
\hline GW190425 & $\begin{array}{c}\text { IMRPhenomD_NRTidal-HS, IMRPhenomD_NRTidal-LS, IMRPhenomPv2_NRTidal-HS, } \\
\text { IMRPhenomPv2_NRTidal-LS, SEOBNRv4T_surrogate_HS, SEOBNRv4T_surrogate_LS, } \\
\text { TEOBResumS-HS, TEOBResumS-LS, TaylorF2-HS, TaylorF2-LS }\end{array}$ \\
\hline GW190426_152155 & IMRPhenomNSBH, IMRPhenomPv2, SEOBNRv4PHM, SEOBNRv4_ROM_NRTidalv2_NSBH, TaylorF2 \\
\hline GW190503_185404 & IMRPhenomD, IMRPhenomPv2, NRSur7dq4, SEOBNRv4P \\
\hline GW190512_180714 & IMRPhenomD, IMRPhenomPv2, SEOBNRv4P, SEOBNRv4PHM \\
\hline GW190513_205428 & IMRPhenomD, IMRPhenomPv2, NRSur7dq4, SEOBNRv4P, SEOBNRv4PHM \\
\hline GW190514_065416 & IMRPhenomD, IMRPhenomPv2, NRSur7dq4, SEOBNRv4P \\
\hline GW190517_055101 & IMRPhenomD, IMRPhenomPv2, NRSur7dq4, SEOBNRv4P, SEOBNRv4PHM \\
\hline GW190519_153544 & IMRPhenomD, IMRPhenomPv2, NRSur7dq4, SEOBNRv4P, SEOBNRv4PHM \\
\hline GW190521 & IMRPhenomPv3HM, NRSur7dq4, SEOBNRv4PHM \\
\hline GW190521_074359 & IMRPhenomD, IMRPhenomPv2, NRSur7dq4, SEOBNRv4P, SEOBNRv4PHM \\
\hline GW190527_092055 & IMRPhenomD, IMRPhenomPv2, NRSur7dq4, SEOBNRv4P \\
\hline GW190602_175927 & IMRPhenomD, IMRPhenomPv2, NRSur7dq4, SEOBNRv4P, SEOBNRv4PHM \\
\hline GW190620_030421 & IMRPhenomD, IMRPhenomPv2, NRSur7dq4, SEOBNRv4P, SEOBNRv4PHM \\
\hline GW190630_185205 & IMRPhenomD, IMRPhenomPv2, NRSur7dq4, SEOBNRv4P, SEOBNRv4PHM \\
\hline GW190701_203306 & IMRPhenomD, IMRPhenomPv2, NRSur7dq4, SEOBNRv4P, SEOBNRv4PHM \\
\hline GW190706_222641 & IMRPhenomD, IMRPhenomPv2, NRSur7dq4, SEOBNRv4P, SEOBNRv4PHM \\
\hline GW190707_093326 & IMRPhenomD, IMRPhenomPv2, SEOBNRv4P, SEOBNRv4PHM \\
\hline GW190708_232457 & IMRPhenomD, IMRPhenomPv2, SEOBNRv4P, SEOBNRv4PHM \\
\hline GW190719_215514 & IMRPhenomD, IMRPhenomPv2, NRSur7dq4, SEOBNRv4P, SEOBNRv4PHM \\
\hline GW190720_000836 & IMRPhenomD, IMRPhenomPv2, SEOBNRv4P, SEOBNRv4PHM \\
\hline GW190727_060333 & IMRPhenomD, IMRPhenomPv2, NRSur7dq4, SEOBNRv4P \\
\hline GW190728_064510 & IMRPhenomD, IMRPhenomPv2, SEOBNRv4P, SEOBNRv4PHM \\
\hline GW190731_140936 & IMRPhenomD, IMRPhenomPv2, NRSur7dq4, SEOBNRv4P \\
\hline GW190803_022701 & IMRPhenomD, IMRPhenomPv2, NRSur7dq4, SEOBNRv4P \\
\hline GW190814 & $\begin{array}{l}\text { IMRPhenomD, IMRPhenomHM, IMRPhenomNSBH, IMRPhenomPv3HM, SEOBNRv4HM_ROM, } \\
\text { SEOBNRv4PHM, SEOBNRv4_ROM, SEOBNRv4_ROM_NRTidalv2_NSBH }\end{array}$ \\
\hline GW190828_063405 & IMRPhenomD, IMRPhenomPv2, NRSur7dq4, SEOBNRv4P, SEOBNRv4PHM \\
\hline GW190828_065509 & IMRPhenomD, IMRPhenomPv2, SEOBNRv4P, SEOBNRv4PHM \\
\hline GW190909_114149 & IMRPhenomD, IMRPhenomPv2, SEOBNRv4P, SEOBNRv4PHM \\
\hline GW190910_112807 & IMRPhenomD, IMRPhenomPv2, SEOBNRv4P, SEOBNRv4PHM \\
\hline GW190915_235702 & IMRPhenomD, IMRPhenomPv2, SEOBNRv4P, SEOBNRv4PHM \\
\hline GW190924_021846 & IMRPhenomD, IMRPhenomPv2, SEOBNRv4P, SEOBNRv4PHM \\
\hline GW190929_012149 & IMRPhenomD, IMRPhenomPv2, SEOBNRv4P, SEOBNRv4PHM \\
\hline GW190930_133541 & IMRPhenomD, IMRPhenomPv2, SEOBNRv4P, SEOBNRv4PHM \\
\hline
\end{tabular}

to use when comparing posterior distributions of parameter estimation runs.

Both of these issues are addressed by the JS divergence [280], which is a smoothed and symmetrized version of $D_{\mathrm{KL}}$. It is defined as

$$
D_{\mathrm{JS}}(p, q) \equiv \frac{1}{2}\left[D_{\mathrm{KL}}(p \mid s)+D_{\mathrm{KL}}(q \mid s)\right]
$$

where $s=(p+q) / 2$ is the average distribution. By construction, the JS divergence is symmetric, and it satisfies $0 \leq D_{\mathrm{JS}} \leq 1$ bit, which follows from

$$
\begin{aligned}
D_{\mathrm{KL}}(p \mid s) & =\int p(\vartheta) \log _{2}\left(\frac{2 p(\vartheta)}{p(\vartheta)+q(\vartheta)}\right) d \vartheta \\
& \leq \int p(\vartheta) \log _{2}\left(\frac{2 p(\vartheta)}{p(\vartheta)}\right) d \vartheta=1 \mathrm{bit} .
\end{aligned}
$$


The convenient properties of the JS divergence make it a useful measure of the difference between posterior probability distributions, and we adopt it to quantify the systematics in our results resulting from different analysis choices.

We use the JS divergence to investigate two sources of systematics that can impact the results of inference: the differences in the models with equivalent physics due to modeling choices and differences due to the inclusion of different physical effects. To assess the importance of the former, we compare the results using our two fiducial precessing waveform models (IMRPhenomPv2 and SEOBNRv4P). For the latter, we consider inclusion of HMs beyond the dominant quadrupole by comparing SEOBNRv4P to models that include HMs: SEOBNRv4PHM and NRSur7dq4. We compute the JS divergence between one-dimensional marginal distributions on two key parameters: mass ratio and effective inspiral spin. We use a threshold of 0.007 bit to deem the differences significant, which for a Gaussian corresponds to a $20 \%$ shift in the mean, measured in units of one standard deviation. This threshold is larger than 0.002 bit, which is the variation that is shown to arise due to stochastic sampling [234]. In the following subsection, we describe how we use our computed JS divergences and JS threshold to choose the default parameter estimation results shown in this work.

\begin{tabular}{|c|c|c|c|c|}
\hline$\star$ & GW190408_181802 & GW190514_065416 & GW190706_222641 & GW190828_063405 \\
\hline$>$ & GW190412 & GW190517_055101 & GW190707_093326 & $28 \_065509$ \\
\hline - & GW190413_052954 & GW190519_153544 & GW190708_232457 & 0909_114149 \\
\hline 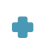 & GW190413_134308 & GW190521_074359 & GW190719_215514 & ㅁ GW190910_112807 \\
\hline च & GW190421_213856 & A GW190527_092055 & GW190720_000836 & $\checkmark \mathrm{GW}$ \\
\hline 4 & GW190424_180648 & GW190602_175927 & GW190727_060333 & GW190924_021846 \\
\hline ○ & GW190503_185404 & GW190620_030421 & GW190728_064510 & GW190929_012149 \\
\hline 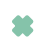 & GW190512_180714 & GW190630_185205 & GW190731_140936 & - GW190930_133541 \\
\hline$\star$ & GW190513_205428 & GW190701_203306 & GW190803_022701 & \\
\hline
\end{tabular}
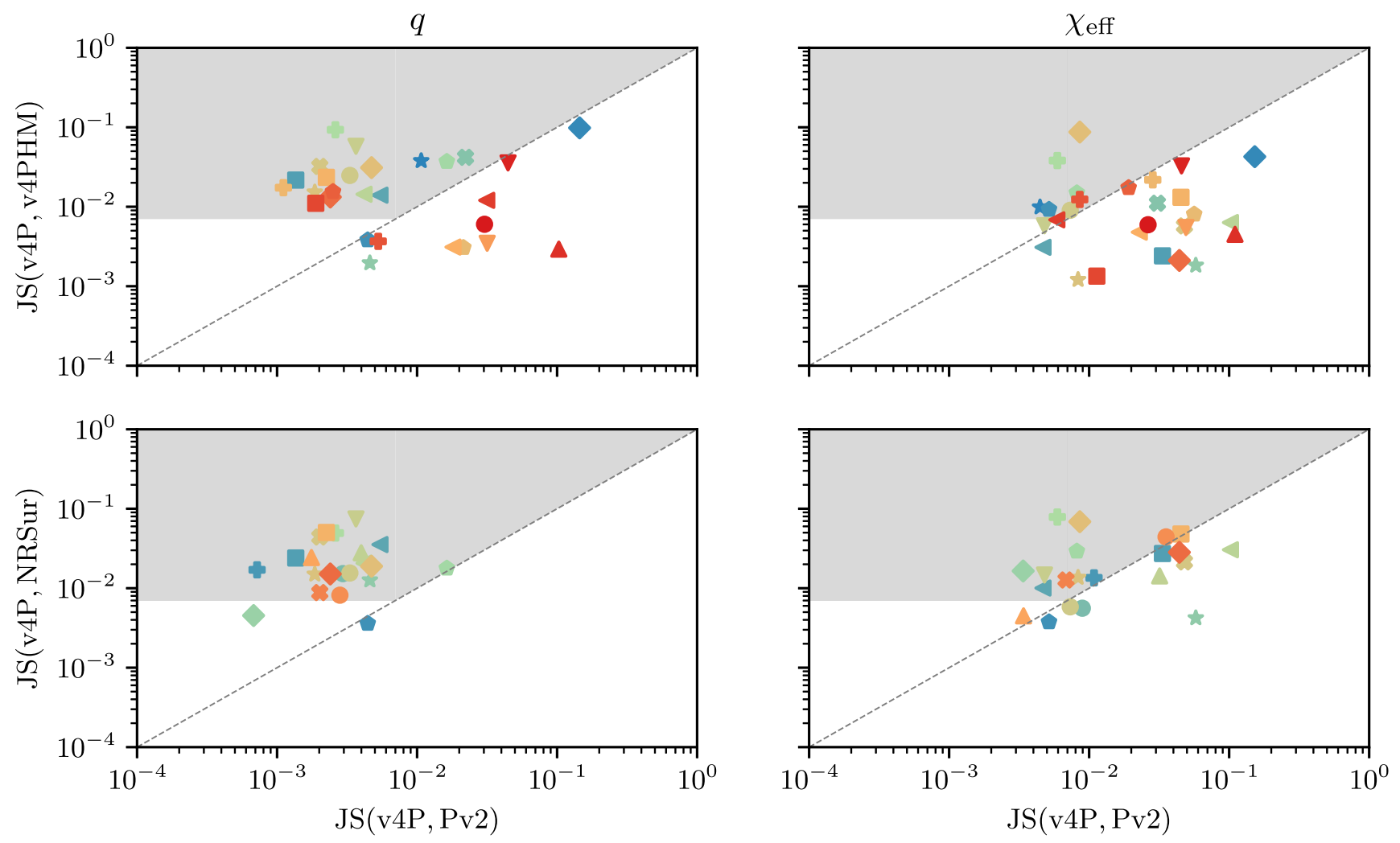

FIG. 13. Jensen-Shannon divergence $D_{\text {JS }}$ of one-dimensional marginal distributions using different waveform models. The vertical axes show $D_{\text {JS }}$ between one-dimensional posteriors using SEOBNRv4P and either SEOBNRv4PHM or NRSur7dq4. The horizontal axes show $D_{\text {JS }}$ between one-dimensional posteriors using SEOBNRv4P and IMRPhenomPv2. The gray regions show the selection criteria from Eq. (A4a): Any events in these regions are presented using higher-order multipole moment results in Sec. VII. 


\section{Choice of waveform models for each event}

We use the above considerations to select which waveform model(s) are used as the fiducial results presented in Sec. VII. [304] In particular, we present results from models with HMs if the following conditions are satisfied for any of the three key parameters and any of the HM models:

$$
\begin{gathered}
D_{\mathrm{JS}}(\text { SEOBNRv4P, HM }) \\
>D_{\mathrm{JS}}(\text { SEOBNRv4P, IMRPhenomPv2 }), \\
D_{\mathrm{JS}}(\text { SEOBNRv4P, HM })>0.007 \text { bit. }
\end{gathered}
$$

When the conditions do not hold, we combine equal number of samples from the results of IMRPhenomPv2 and SEOBNRv4P and use the joint samples. The only exceptions are GW190425 and GW190426_152155, for which we use the tidal waveforms described in Sec. V, and GW190412, GW190521, and GW190814, for which we know HMs are significant $[33,34,236]$. Figure 13 shows the JS divergences for every event (except GW190425, GW190426_152155, GW190521, and GW190814) to compare the effects of model systematics versus the effects of HMs. The gray regions correspond to the criteria in Eq. (A4a): Any events with $D_{\text {JS }}$ values in those regions are presented using HM results in Sec. VII.

\section{Waveform comparison-Model systematics}

The in-depth studies of GW190412, GW190521, and GW190814 quantitatively demonstrate how much the choice of gravitational waveform impacts our interpretation of real gravitational-wave events. We identify several additional instances where analyses with our two fiducial precessing waveform models (IMRPhenomPv2 and SEOBNRv4P) have notable differences, as measured by the JS divergence.

To illustrate the impact of systematics, we show in Fig. 14 the posterior distributions for the events with extremal JS divergence in the key parameters $q$ and $\chi_{\text {eff }}$ : GW190924_021846 and GW190521_074359. For GW190924_021846, SEOBNRv4P prefers more moderate mass ratios around $1 / 2$ and shows only minor differences in the other parameters. Meanwhile, GW190521_074359 shows the largest differences in the effective inspiral spin, with SEOBNRv4P more clearly preferring nonzero values.

While the cases above demonstrate the moderate shifts that result from model systematics, for all the events presented in this paper, there is always substantial overlap between the posteriors for all the key quantities we consider. Therefore, systematic uncertainty remains subdominant to statistical uncertainty.

\section{Waveform comparison-Effect of higher-order multipole moments modes}

Previous investigations of GW190412, GW190521, and GW190814 show that gravitational-wave radiation beyond the quadrupole can significantly impact our inferences on individual events [33,34,236]. Incorporating nonquadrupole modes enables tighter inferences on the source's distance, component masses, and spins [211,301, 305-317]. HMs impact inferences about even shortduration and low-amplitude sources because of the weaker constraints our observations provide. For example, including nonquadrupole modes can tighten constraints on the source mass ratio for low-amplitude sources [305,306],

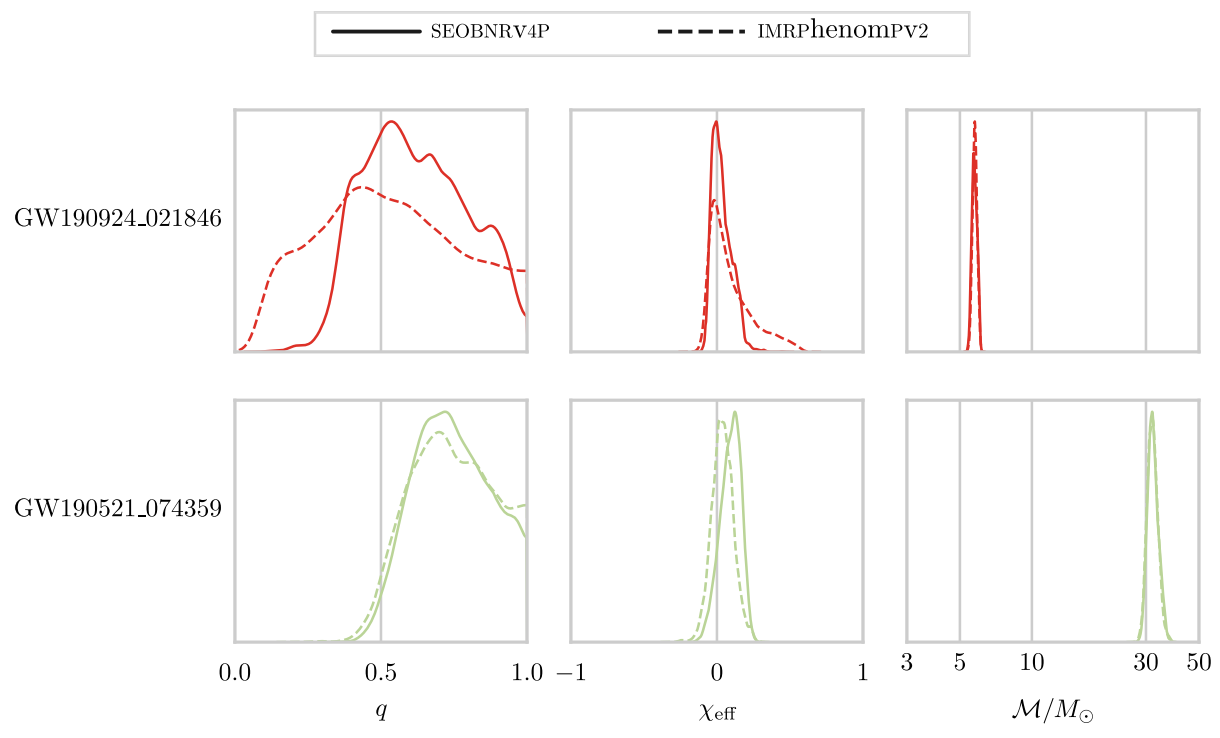

FIG. 14. Marginal posterior distributions on the mass ratio $q$, effective inspiral spin $\chi$ eff, and source-frame chirp mass $\mathcal{M}$ for GW190924_021846 and GW190521_074359, with the IMRPhenomPv2 and SEOBNRv4P waveform families. 
because high-mass ratio sources more efficiently produce nonquadrupole modes.

We systematically apply one or two models with HM to all sources in our catalog. We find that a majority of the sources investigated have modest shifts in mass ratio or $\chi_{\text {eff }}$ due to the impact of HMs.

To illustrate the impact of nonquadrupole modes, Fig. 15 shows posterior inferences on GW190519_ 153544, GW190602_175927, GW190706_222641, and GW190929_012149 using one or two independent models which include nonquadrupole modes (SEOBNRv4PHM and NRSur7dq4). Using more complete waveform models, the source of GW190519_153544 is inferred to be edge on, at a smaller distance, a more asymmetric mass ratio and, thus, a higher source-frame mass $m_{1}$. Because of this source's favorable orientation, nonquadrupole modes have a significant impact, with a Bayes factor for HMs of approximately 15. Similarly, nonquadrupole modes allow us to more strongly exclude both extreme and comparable mass ratios for the source responsible for GW190929_012149 and to disfavor comparable masses for GW190706_222641. Conversely, using the same two waveform models, the high-mass source responsible for GW190602_175927 is more confidently inferred to have mass ratio $q$ closer to unity, and, therefore, $m_{2}$ is skewed to larger values. Nonquadrupole modes also have a noticeable impact on parameters of GW190630_185205 and GW190828_ 065509 , in particular, the mass ratio and luminosity distance.

\section{APPENDIX B: WAVEFORM CONSISTENCY TESTS}

There are several different quantitative measures that can be used to measure waveform consistency. These include the residual SNR, which is found by subtracting the best-fit waveform template $h_{T}$ from the data $d$ and then applying minimally modeled methods to search for any coherent excess in the noise residual $r=d-h_{T}$. Additional measures of waveform consistency include the distance between waveforms, $\Delta^{2}\left\langle h_{1}, h_{2}\right\rangle=\left\langle h_{1}-h_{2} \mid h_{1}-h_{2}\right\rangle$, and the match, or overlap, $\mathcal{O}\left\langle h_{1}, h_{2}\right\rangle$.

The residuals test is applied as a test of general relativity $[9,10]$, at least within the precision with which the waveform models approximate general relativity. Distance and match provide more sensitive measures of the waveform consistency than the residuals test, since the extrinsic parameters of the source, such as the arrival time, sky location, and polarization, are constrained by the full signal, while for the residuals test the extrinsic parameters have to be constrained from the (usually small) difference between the signal and the template. To compare the signal reconstructions for the current catalog of sources, we adopt the waveform match as our measure of waveform consistency, since it does not depend on the overall amplitude of the signals, making it a convenient choice when comparing events with a range of amplitudes.

To make a quantitative assessment of the waveform match values, we need to know how the match depends on quantities such as the SNR and time-frequency volume of the signals. Instrument noise leads to nonzero mismatches, $\mathrm{MM}=1-\mathcal{O}$, even when using perfect templates. For example, the maximum likelihood solution for a perfect template has a mismatch with a mean and variance given by [318]

$$
E[\mathrm{MM}] \simeq \frac{D-1}{2 \mathrm{SNR}^{2}}, \quad \operatorname{Var}[\mathrm{MM}] \simeq \frac{D-1}{2 \mathrm{SNR}^{4}},
$$

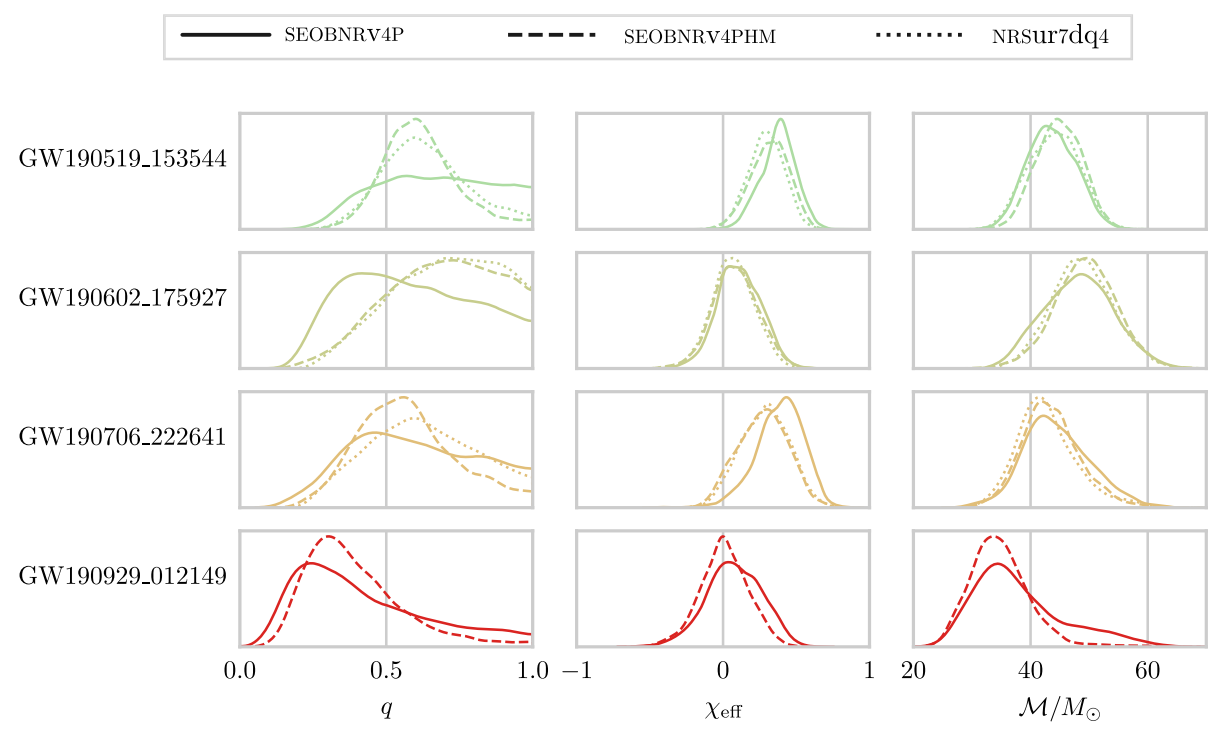

FIG. 15. Marginal posterior distributions on mass ratio $q$, effective inspiral spin $\chi$ eff, and source-frame chirp mass $\mathcal{M}$, for GW190519_153544, GW190602_175927, GW190706_222641, and GW190929_012149 with the SEOBNRv4P, SEOBNRv4PHM, and NRSur7dq4 waveform families. 
where $D$ is the number of parameters that define the signal model and where the reduction from $D$ to $D-1$ is because the match is independent from the overall amplitude of the signal. For the minimally modeled waveform reconstructions, the distributions of match values are more difficult to predict. Using simulations, it is found that the mismatch decreases with SNR, but more slowly than for templates, since the effective dimension of the model increases with SNR [108]. The mismatch also scales with the timefrequency volume. For binary systems of a given SNR, the mismatch is generally smaller for high-mass systems $[108,319,320]$. Given these complexities, we choose to empirically estimate the match distribution from simulations for each event. As a proxy for the signal, we use fair draws from the on-source template-based analysis and inject these into data surrounding the event; the right ascensions for the simulated signals are adjusted such that the simulated source is at the same sky location in the frame of the detectors. For the majority of events, the waveform model used for the injections is IMRPhenomPv2.
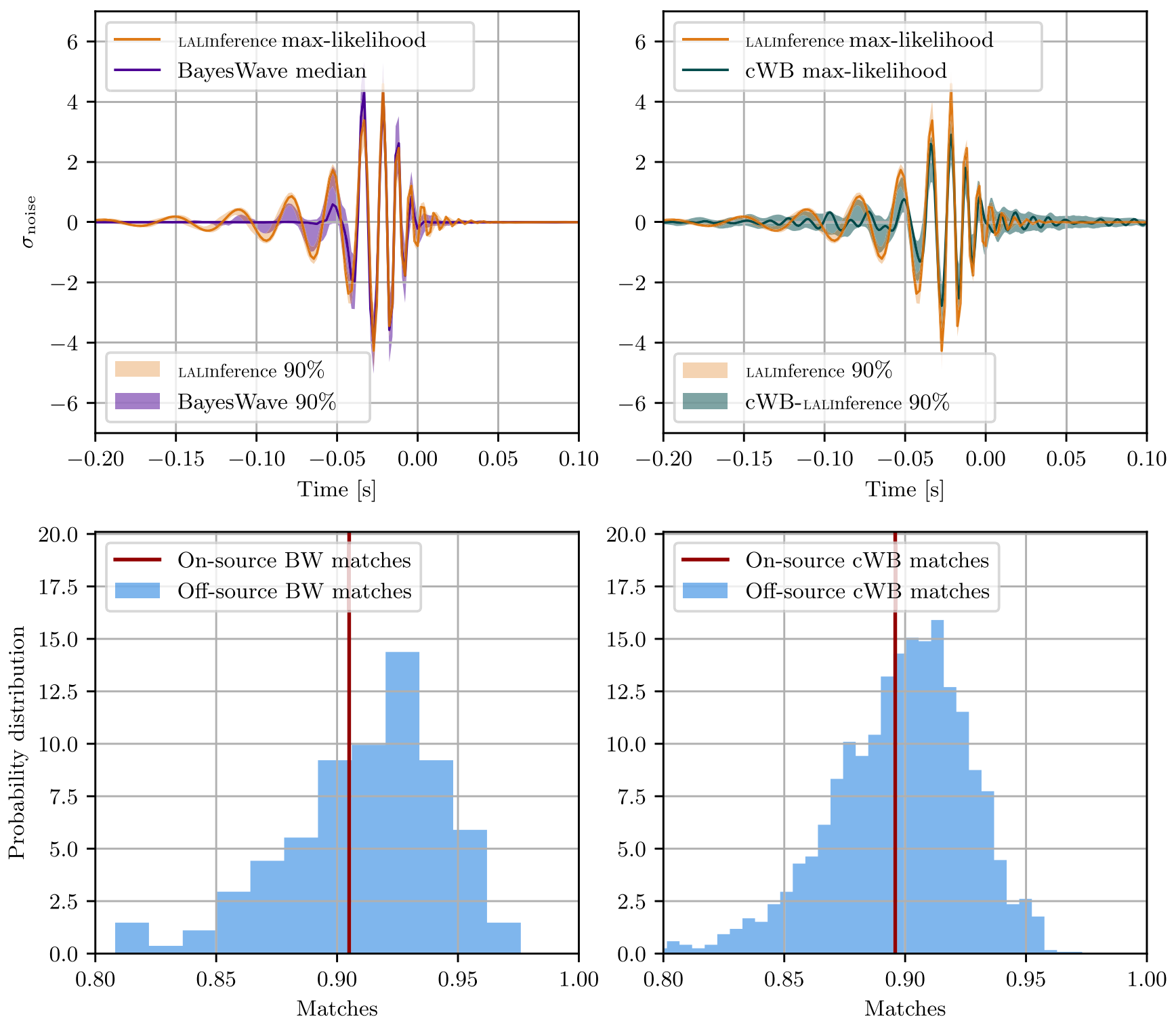

FIG. 16. The upper panels show waveform reconstructions for GW190519_153544 in the LIGO Livingston detector. The waveform posterior from the template-based analysis (shown in orange) is compared to the BW analysis in the upper left and to the cWB analysis in the upper right. The panels also show the $90 \%$ credible bands for the Bayesian LALInference and BW algorithms and the 90\% confidence band for cWB derived from off-source injections, i.e., by injecting samples from the template-based analysis into data surrounding the event and repeating the analysis multiple times (these bands are computed on an individual time sample basis). The lower two panels show the distribution of overlap values when running BW and cWB on waveforms drawn from the template-based analysis that are injected into data surrounding the event. The fraction of runs with matches below that of the on-source analysis give the $p$ value for the event. 
The exceptions are GW190412, GW190521, and GW190814, where IMRPhenomPv3HM is used.

The cWB and BW algorithms are used to produce point estimates for the waveform reconstructions for each of the simulated events. For cWB the point estimate is a constrained maximum likelihood reconstruction, while for BW the point estimate is the median of the waveform posterior distribution. Figure 16 illustrates the results obtained for GW190519_153544. The upper panels compare the template-based LALInference waveform reconstruction in the LIGO Livingston detector to the minimally modeled BW and cWB reconstructions. The solid lines are point estimates for the waveforms: for LALInference the maximum likelihood; for BW the median of posterior draws; and for cWB the constrained maximum likelihood. The panels also show the $90 \%$ credible bands for the Bayesian LALInference and BW algorithms and the $90 \%$ confidence band for cWB derived by injecting samples from the template-based analysis into data surrounding the event and repeating the analysis multiple times (these bands are computed on an individual time sample basis). The lower panels in Fig. 16 show the distribution of overlaps found when running $\mathrm{BW}$ and $\mathrm{cWB}$ on simulated data with similar properties to the event. Waveforms drawn from the onsource LALInference analysis are injected into data surrounding the event. The overlap between the injected waveform and the point estimates from the BW and cWB analyses of these injections are then used to produce the histograms seen in Fig. 16. The distribution of the match values defines a null distribution for each detected event, which takes into account the variability of the LALInference posterior distribution, the fluctuations of the detector noise, and the waveform reconstruction errors. The fraction of off-source analyses with overlaps below the on-source match values, which are shown as vertical lines in the lower panels in Fig. 16, defines the $p$ value for this event.

The same analysis procedure is repeated for a subset of additional events. cWB uses only events that are above the cWB search thresholds (resulting in a morphologydependent SNR threshold which is about 7-10 for the events reported in this catalog), while for BW the analysis is restricted to events where the on-source BW analysis yields SNR $>7$. Figure 17 shows the on-source match values vs the off-source median match values with $90 \%$ intervals. The upper panel shows the results of the BW analysis, while the lower panel shows the results of the cWB analysis. In both cases, the $p$ values point to a good agreement between the minimally modeled and templatebased reconstructions.

The discrepancies between the two plots may be ascribed to different choices made in the two reconstruction algorithms. cWB is both a detection and a reconstruction pipeline. For this reason, reconstructions are performed with the same production settings used in searches.

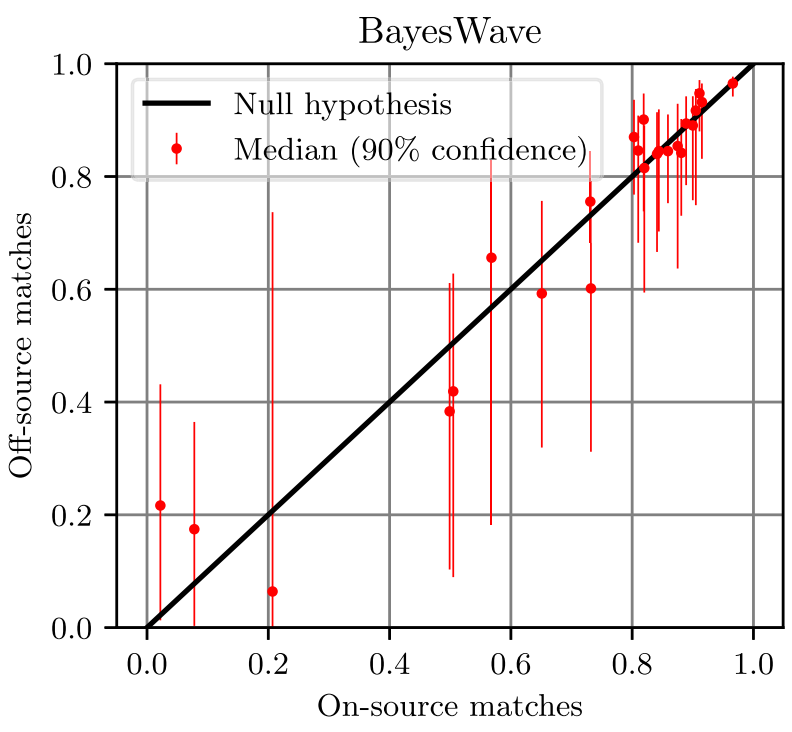

coherent WaveBurst

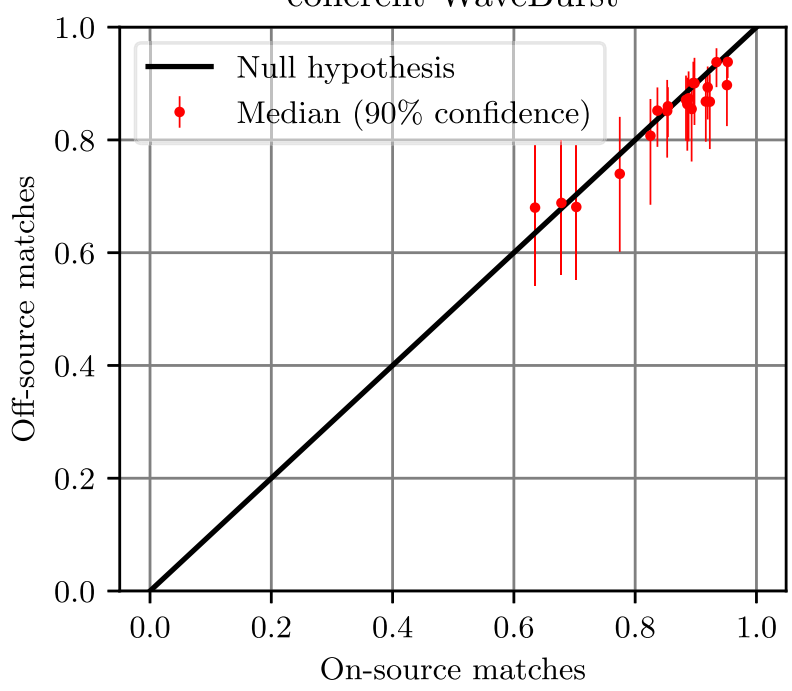

FIG. 17. Off-source vs on-source match values for the candidate events in O3a. The upper panel displays the results of the $\mathrm{BW}$ analysis, and the lower panel shows the results of the cWB analysis. The on-source match on the horizontal axis is the value obtained comparing the maximum likelihood waveform from parameter estimation with point estimates from the minimally modeled waveform reconstructions. The off-source match on the vertical axis is the median value of the match distribution obtained from off-source injection of sample waveforms from the template-based posterior distribution. In both panels, the errors bars denote the $90 \%$ equal-tailed confidence interval.

The production settings are optimized for noise rejection and to enforce strong network coherency constraints. To construct the match distributions, cWB uses about 20003000 waveform injections per event; however, those injections that are below the cWB thresholds are not reconstructed: In the majority of cases reported here (13), the reconstruction efficiency is greater than $80 \%$, while in a few other cases (7), the efficiency ranges from 
$15 \%$ to $50 \%$. For each event, this efficiency depends on the variability of the noise background. In cases of lower efficiency, we also check that the waveforms that are successfully reconstructed by cWB have parameter distributions that are statistically indistinguishable from those of the injected waveforms.

BW employs Bayesian inference to characterize detections made by the $\mathrm{CBC}$ and cWB search pipelines. As such, no cuts are made on the waveform reconstructions, which means that, for quiet signals, some of the samples are drawn from the prior distribution, resulting in a wide spread in the match distributions.

The distribution of match values for each event are used to compute $p$ values. The overall consistency of the template-based and minimally modeled waveform reconstructions can then be summarized by plotting the $p$ values, ranked in ascending order, against the theoretical distribution. In such plots, any significant deviations below the plot diagonal point to events that should undergo further analysis. The $p$ values for the events reconstructed by cWB and BW are shown in Fig. 12 in the main text. Applying the Fisher test [321] to the ensemble of $p$ values yields combined $p$ values of 0.57 for the $\mathrm{BW}$ analysis and 0.99 for the cWB analysis, indicating that there is no reason to reject the null hypothesis that the template-based and minimally modeled analysis are in agreement. The Fisher test is one sided in that it penalizes only $p$ values that are lower than expected. The cWB analysis includes instances where the $p$ values are higher than predicted, indicating that the on-source matches are higher than expected based on the off-source distributions. The cause of this bias is likely due to an asymmetry between the on-source and off-source analyses. The onsource analysis computes the match between the maximum likelihood template-based waveform and its reconstruction, while the off-source analysis computes the match between the injected template and its reconstruction. Ideally, the analysis would be symmetric, with the maximum likelihood template used both onsource and off-source, but the computational cost of running the full template-based analysis on the thousands of off-source injections is prohibitively expensive.

\section{APPENDIX C: COSMOLOGICAL DISTANCE RESAMPLING}

For the luminosity distance, a prior that goes as $D_{L}^{2}$ is enforced in the sampling, following the same procedure as described in previous publications. As this assumption becomes increasingly unrealistic as events are detected at greater distances, the posterior distributions shown in this paper are derived from a physically motivated prior that incorporates cosmological effects. We perform rejection sampling on the initial posterior samples to instead use a prior corresponding to a uniform merger rate per comoving volume in the rest frame of the source. Using a standard flat
$\Lambda \mathrm{CDM}$ cosmology, samples are accepted according to the weight

$$
\begin{aligned}
w(z) & \propto \frac{1}{(1+z)} \frac{d V_{c}}{d V_{E}} \\
& \propto(1+z)^{-2}\left(\frac{D_{L} E(z)}{D_{H}}+(1+z)^{2}\right)^{-1} .
\end{aligned}
$$

The initial $1 /(1+z)$ factor accounts for time dilation of the observed merger rate, $d V_{c}$ is the comoving volume element, and $d V_{E}=D_{L}^{2} d \Omega$ is the Euclidean volume element. $D_{H}=c / H_{0}$ is the Hubble distance and $E(z) \simeq$ $\sqrt{\Omega_{m}(1+z)^{3}+\Omega_{\Lambda}}$ for $\Lambda \mathrm{CDM}$, and we use Hubble constant $H_{0}=67.9 \mathrm{~km} \mathrm{~s}^{-1} \mathrm{Mpc}^{-1}$ and matter density $\Omega_{m}=0.3065=1-\Omega_{\Lambda}[238]$.

[1] B. P. Abbott et al. (LIGO Scientific Collaboration, Virgo Collaboration), Observation of Gravitational Waves from a Binary Black Hole Merger, Phys. Rev. Lett. 116, 061102 (2016).

[2] J. Aasi et al. (LIGO Scientific Collaboration), Advanced LIGO, Classical Quantum Gravity 32, 074001 (2015).

[3] F. Acernese et al. (Virgo Collaboration), Advanced Virgo: A Second-Generation Interferometric Gravitational Wave Detector, Classical Quantum Gravity 32, 024001 (2015).

[4] B. P. Abbott et al. (LIGO Scientific Collaboration, Virgo Collaboration), GW151226: Observation of Gravitational Waves from a 22-Solar-Mass Binary Black Hole Coalescence, Phys. Rev. Lett. 116, 241103 (2016).

[5] B. P. Abbott et al. (LIGO Scientific Collaboration, Virgo Collaboration), GW170104: Observation of a 50-SolarMass Binary Black Hole Coalescence at Redshift 0.2, Phys. Rev. Lett. 118, 221101 (2017); Erratum, Phys. Rev. Lett. 121, 129901 (2018).

[6] B. P. Abbott et al. (LIGO Scientific Collaboration, Virgo Collaboration), GW170608: Observation of a 19-SolarMass Binary Black Hole Coalescence, Astrophys. J. 851, L35 (2017).

[7] B. P. Abbott et al. (LIGO Scientific Collaboration, Virgo Collaboration), GW170814: A Three-Detector Observation of Gravitational Waves from a Binary Black Hole Coalescence, Phys. Rev. Lett. 119, 141101 (2017).

[8] B. P. Abbott et al. (LIGO Scientific Collaboration, Virgo Collaboration), GWTC-1: A Gravitational-Wave Transient Catalog of Compact Binary Mergers Observed by LIGO and Virgo during the First and Second Observing Runs, Phys. Rev. X 9, 031040 (2019).

[9] B. P. Abbott et al. (LIGO Scientific Collaboration, Virgo Collaboration), Tests of General Relativity with GW150914, Phys. Rev. Lett. 116, 221101 (2016); Erratum, Phys. Rev. Lett. 121, 129902 (2018).

[10] B. P. Abbott et al. (LIGO Scientific Collaboration, Virgo Collaboration), Tests of General Relativity with the Binary Black Hole Signals from the LIGO-Virgo Catalog GWTC-1, Phys. Rev. D 100, 104036 (2019). 
[11] B. P. Abbott et al. (LIGO Scientific Collaboration, Virgo Collaboration), Binary Black Hole Population Properties Inferred from the First and Second Observing Runs of Advanced LIGO and Advanced Virgo, Astrophys. J. 882, L24 (2019).

[12] B. P. Abbott et al. (LIGO Scientific Collaboration, Virgo Collaboration), GW170817: Observation of Gravitational Waves from a Binary Neutron Star Inspiral, Phys. Rev. Lett. 119, 161101 (2017).

[13] B. P. Abbott et al. (LIGO Scientific Collaboration, Virgo Collaboration, Fermi-GBM, INTEGRAL), Gravitational Waves and Gamma-Rays from a Binary Neutron Star Merger: GW170817 and GRB 170817A, Astrophys. J. 848, L13 (2017).

[14] B. P. Abbott et al. (LIGO Scientific Collaboration, Virgo Collaboration, Fermi GBM, INTEGRAL, IceCube, AstroSat Cadmium Zinc Telluride Imager Team, IPN, InsightHxmt, ANTARES, Swift, AGILE Team, 1M2H Team, Dark Energy Camera GW-EM, DES, DLT40, GRAWITA, Fermi-LAT, ATCA, ASKAP, Las Cumbres Observatory Group, OzGrav, DWF (Deeper Wider Faster Program), AST3, CAASTRO, VINROUGE, MASTER, J-GEM, GROWTH, JAGWAR, CaltechNRAO, TTU-NRAO, NuSTAR, Pan-STARRS, MAXI Team, TZAC Consortium, KU, Nordic Optical Telescope, ePESSTO, GROND, Texas Tech University, SALT Group, TOROS, BOOTES, MWA, CALET, IKI-GW Follow-up, H.E.S.S., LOFAR, LWA, HAWC, Pierre Auger, ALMA, Euro VLBI Team, Pi of Sky, Chandra Team at McGill University, DFN, ATLAS Telescopes, High Time Resolution Universe Survey, RIMAS, RATIR, SKA South Africa/MeerKAT), Multimessenger Observations of a Binary Neutron Star Merger, Astrophys. J. 848, L12 (2017).

[15] B. P. Abbott et al. (LIGO Scientific Collaboration, Virgo Collaboration), GW170817: Measurements of Neutron Star Radii and Equation of State, Phys. Rev. Lett. 121, 161101 (2018).

[16] B. P. Abbott et al. (LIGO Scientific Collaboration, Virgo Collaboration), Model Comparison from LIGO-Virgo Data on GW170817's Binary Components and Consequences for the Merger Remnant, Classical Quantum Gravity 37, 045006 (2020).

[17] B. P. Abbott et al. (LIGO Scientific Collaboration, Virgo Collaboration, 1M2H, Dark Energy Camera GW-E, DES, DLT40, Las Cumbres Observatory, VINROUGE, MASTER), A Gravitational-Wave Standard Siren Measurement of the Hubble Constant, Nature (London) 551, 85 (2017).

[18] B. P. Abbott et al. (LIGO Scientific Collaboration, Virgo Collaboration), A Gravitational-Wave Measurement of the Hubble Constant Following the Second Observing Run of Advanced LIGO and Virgo, Astrophys. J. 909, 218 (2021).

[19] B. P. Abbott et al. (LIGO Scientific Collaboration, Virgo Collaboration), Tests of General Relativity with GW170817, Phys. Rev. Lett. 123, 011102 (2019).

[20] R. Abbott et al. (LIGO Scientific Collaboration, Virgo Collaboration), Open Data from the First and Second Observing Runs of Advanced LIGO and Advanced Virgo, SoftwareX 3, 100658 (2021).
[21] A. Trovato (LIGO Scientific Collaboration, Virgo Collaboration), GWOSC: Gravitational Wave Open Science Center, Proc. Sci., Asterics2019 (2020) 082.

[22] B. Zackay, L. Dai, T. Venumadhav, J. Roulet, and M. Zaldarriaga, Detecting Gravitational Waves with Disparate Detector Responses: Two New Binary Black Hole Mergers, arXiv:1910.09528.

[23] T. Venumadhav, B. Zackay, J. Roulet, L. Dai, and M. Zaldarriaga, New Binary Black Hole Mergers in the Second Observing Run of Advanced LIGO and Advanced Virgo, Phys. Rev. D 101, 083030 (2020).

[24] T. Venumadhav, B. Zackay, J. Roulet, L. Dai, and M. Zaldarriaga, New Search Pipeline for Compact Binary Mergers: Results for Binary Black Holes in the First Observing Run of Advanced LIGO, Phys. Rev. D 100, 023011 (2019).

[25] B. Zackay, T. Venumadhav, L. Dai, J. Roulet, and M. Zaldarriaga, Highly Spinning and Aligned Binary Black Hole Merger in the Advanced LIGO First Observing Run, Phys. Rev. D 100, 023007 (2019).

[26] A. H. Nitz, C. Capano, A. B. Nielsen, S. Reyes, R. White, D. A. Brown, and B. Krishnan, 1-OGC: The First Open Gravitational-Wave Catalog of Binary Mergers from Analysis of Public Advanced LIGO Data, Astrophys. J. 872, 195 (2019).

[27] A. H. Nitz, T. Dent, G. S. Davies, S. Kumar, C. D. Capano, I. Harry, S. Mozzon, L. Nuttall, A. Lundgren, and M. Tápai, 2-OGC: Open Gravitational-Wave Catalog of Binary Mergers from Analysis of Public Advanced LIGO and Virgo Data, Astrophys. J. 891, 123 (2019).

[28] R. Magee et al., Sub-threshold Binary Neutron Star Search in Advanced LIGO's First Observing Run, Astrophys. J. 878, L17 (2019).

[29] B. Allen, W. G. Anderson, P. R. Brady, D. A. Brown, and J. D. E. Creighton, FINDCHIRP: An Algorithm for Detection of Gravitational Waves from Inspiraling Compact Binaries, Phys. Rev. D 85, 122006 (2012).

[30] H.-Y. Chen, D. E. Holz, J. Miller, M. Evans, S. Vitale, and J. Creighton, Distance Measures in Gravitational-Wave Astrophysics and Cosmology, Classical Quantum Gravity 38, 055010 (2021).

[31] LIGO Scientific Collaboration and Virgo Collaboration, GCN 25871, 25829, 25753, 25503, 25497, 25324, 25187, 25164, 25115, 25012, 24998, 24950, 24922, 24717, 24632, 24621, 24598, 24570, 24522, 24503, 24377, 24237, 24168, 24141, 24098, and 24069 (2019), https:// gcn.gsfc.nasa.gov/lvc.html.

[32] B. P. Abbott et al. (LIGO Scientific Collaboration, Virgo Collaboration), GW190425: Observation of a Compact Binary Coalescence with Total Mass 3.4 $M_{\odot}$, Astrophys. J. Lett. 892, L3 (2020).

[33] R. Abbott et al. (LIGO Scientific Collaboration, Virgo Collaboration), GW190814: Gravitational Waves from the Coalescence of a 23 Solar Mass Black Hole with a 2.6 Solar Mass Compact Object, Astrophys. J. 896, L44 (2020).

[34] R. Abbott et al. (LIGO Scientific Collaboration, Virgo Collaboration), GW190521: A Binary Black Hole Merger with a Total Mass of $150 M_{\odot}$, Phys. Rev. Lett. 125, 101102 (2020). 
[35] R. Abbott et al. (LIGO Scientific Collaboration, Virgo Collaboration), Properties and Astrophysical Implications of the $150 M_{\odot}$ Binary Black Hole Merger GW190521, Astrophys. J. Lett. 900, L13 (2020).

[36] LIGO and Virgo Collaborations, Tests of General Relativity with Binary Black Holes from the second LIGOVirgo Gravitational-Wave Transient Catalog, LIGO Report No. DCC P2000091, 2020, https://dcc.ligo.org/ LIGO-P2000091.

[37] LIGO and Virgo Collaborations, Population Properties of Compact Objects from the Second LIGO-Virgo Gravitational-Wave Transient Catalog, LIGO Report No. DCC P2000077, 2020, https://dcc.ligo.org/LIGO-P2000077.

[38] G. Vajente, E. K. Gustafson, and D. H. Reitze, Precision Interferometry for Gravitational Wave Detection: Current Status and Future Trends (Academic, New York, 2019), Chap. 3, pp. 75-148.

[39] B. P. Abbott et al. (LIGO Scientific Collaboration, Virgo Collaboration), Binary Black Hole Mergers in the First Advanced LIGO Observing Run, Phys. Rev. X 6, 041015 (2016); Erratum, Phys. Rev. X 8, 039903 (2018).

[40] A. Buikema et al. (aLIGO Collaboration), Sensitivity and Performance of the Advanced LIGO Detectors in the Third Observing Run, Phys. Rev. D 102, 062003 (2020).

[41] J. C. Driggers et al. (LIGO Scientific Collaboration), Improving Astrophysical Parameter Estimation via Offline Noise Subtraction for Advanced LIGO, Phys. Rev. D 99, 042001 (2019).

[42] G. Billingsley, H. Yamamoto, and L. Zhang, Characterization of Advanced LIGO Core Optics, Proc. Annu. Meet.-Am. Soc. Precis. Eng. 66, 78 (2017).

[43] R. Dolesi, M. Hueller, D. Nicolodi, D. Tombolato, S. Vitale, P. J. Wass, W. J. Weber, M. Evans, P. Fritschel, R. Weiss, J. H. Gundlach, C. A. Hagedorn, S. Schlamminger, G. Ciani, and A. Cavalleri, Brownian Force Noise from Molecular Collisions and the Sensitivity of Advanced Gravitational Wave Observatories, Phys. Rev. D 84, 063007 (2011).

[44] A. Brooks, Point Absorbers in Advanced LIGO, https://alog .ligo-wa.caltech.edu/aLOG/index.php?callRep=34853.

[45] M. Evans et al., Observation of Parametric Instability in Advanced LIGO, Phys. Rev. Lett. 114, 161102 (2015).

[46] S. Biscans, S. Gras, C. D. Blair, J. Driggers, M. Evans, P. Fritschel, T. Hardwick, and G. Mansell, Suppressing Parametric Instabilities in LIGO Using Low-Noise Acoustic Mode Dampers, Phys. Rev. D 100, 122003 (2019).

[47] R. Schnabel, N. Mavalvala, D. E. Mcclelland, and P. K. Lam, Quantum Metrology for Gravitational Wave Astronomy, Nat. Commun. 1, 121 (2010).

[48] L. Barsotti, J. Harms, and R. Schnabel, Squeezed Vacuum States of Light for Gravitational Wave Detectors, Rep. Prog. Phys. 82, 016905 (2019).

[49] M. Tse et al., Quantum-Enhanced Advanced LIGO Detectors in the Era of Gravitational-Wave Astronomy, Phys. Rev. Lett. 123, 231107 (2019).

[50] J. Abadie et al. (LIGO Scientific Collaboration), A Gravitational Wave Observatory Operating beyond the Quantum Shot-Noise Limit: Squeezed Light in Application, Nat. Phys. 7, 962 (2011).
[51] H. Grote, K. Danzmann, K. L. Dooley, R. Schnabel, J. Slutsky, and H. Vahlbruch, First Long-Term Application of Squeezed States of Light in a Gravitational-Wave Observatory, Phys. Rev. Lett. 110, 181101 (2013).

[52] D. J. Ottaway, P. Fritschel, and S. J. Waldman, Impact of Upconverted Scattered Light on Advanced Interferometric Gravitational Wave Detectors, Opt. Express 20, 8329 (2012).

[53] D. Aisa et al., The Advanced Virgo Monolithic Fused Silica Suspension, Nucl. Instrum. Methods Phys. Res., Sect. A 824, 644 (2016).

[54] L. Naticchioni (Virgo Collaboration), The Payloads of Advanced Virgo: Current Status and Upgrades, J. Phys. Conf. Ser. 957, 012002 (2018).

[55] M. Blom, M. G. Beker, A. Bertolini, J. F. J. van den Brand, H. J. Bulten, M. Doets, E. Hennes, F. A. Mul, D. S. Rabeling, and A. Schimmel, Vertical and Horizontal Seismic Isolation Performance of the Advanced Virgo External Injection Bench Seismic Attenuation System, Phys. Procedia 61, 641 (2015).

[56] M. Blom, Seismic Attenuation for Advanced Virgo: Vibration Isolation for the External Injection Bench, Ph.D.thesis, Nikhef, 2015.

[57] In Ref. [58], it is reported that the injected power during $\mathrm{O} 2$ is $14 \mathrm{~W}$; however, measurements taken during end-of-run commissioning activities determined that the correct value is approximately $30 \%$ lower than expected.

[58] F. Acernese et al. (Virgo Collaboration), Calibration of Advanced Virgo and Reconstruction of the Gravitational Wave Signal $h(t)$ during the Observing Run O2, Classical Quantum Gravity 35, 205004 (2018).

[59] A. Rocchi, E. Coccia, V. Fafone, V. Malvezzi, Y. Minenkov, and L. Sperandio, Thermal Effects and Their Compensation in Advanced Virgo, J. Phys. Conf. Ser. 363, 012016 (2012).

[60] I. Nardecchia et al., Integrated Dynamical Thermal Compensation Techniques for Advanced Virgo, in Proceedings of the 2nd Gravitational-Waves Science and Technology Symposium (GRASS 2019), Padova, Italy, 2019 (to be published), https://zenodo.org/record/3567579.

[61] F. Acernese et al. (Virgo Collaboration), Increasing the Astrophysical Reach of the Advanced Virgo Detector via the Application of Squeezed Vacuum States of Light, Phys. Rev. Lett. 123, 231108 (2019).

[62] H. Vahlbruch, M. Mehmet, K. Danzmann, and R. Schnabel, Detection of $15 \mathrm{~dB}$ Squeezed States of Light and Their Application for the Absolute Calibration of Photoelectric Quantum Efficiency, Phys. Rev. Lett. 117, 110801 (2016).

[63] B. P. Abbott et al. (LIGO Scientific Collaboration, Virgo Collaboration), A Guide to LIGO-Virgo Detector Noise and Extraction of Transient Gravitational-Wave Signals, Classical Quantum Gravity 37, 055002 (2020).

[64] R. P. Fisher, G. Hemming, M.-A. Bizouard, D. A. Brown, P. F. Couvares, F. Robinet, and D. Verkindt, DQSEGDB: A Time-Interval Database for Storing Gravitational Wave Observatory Metadata, SoftwareX 14, 100677 (2021).

[65] N. Christensen, P. Shawhan, and G. Gonzalez (LIGO Scientific Collaboration), Vetoes for Inspiral Triggers in LIGO Data, Classical Quantum Gravity 21, S1747 (2004). 
[66] B. P. Abbott et al. (LIGO Scientific Collaboration, Virgo Collaboration), Characterization of Transient Noise in Advanced LIGO Relevant to Gravitational Wave Signal GW150914, Classical Quantum Gravity 33, 134001 (2016).

[67] A. Viets et al., Reconstructing the Calibrated Strain Signal in the Advanced LIGO Detectors, Classical Quantum Gravity 35, 095015 (2018).

[68] F. Acernese et al. (Virgo Collaboration), Calibration of Advanced Virgo and Reconstruction of the Gravitational Wave Signal $h(t)$ during the Observing Run O2, Classical Quantum Gravity 35, 205004 (2018).

[69] B. P. Abbott et al. (LIGO Scientific Collaboration), Calibration of the Advanced LIGO Detectors for the Discovery of the Binary Black-Hole Merger GW150914, Phys. Rev. D 95, 062003 (2017).

[70] D. Tuyenbayev et al., Improving LIGO Calibration Accuracy by Tracking and Compensating for Slow Temporal Variations, Classical Quantum Gravity 34, 015002 (2017).

[71] S. Karki et al., The Advanced LIGO Photon Calibrators, Rev. Sci. Instrum. 87, 114503 (2016).

[72] D. Bhattacharjee, Y. Lecoeuche, S. Karki, J. Betzwieser, V. Bossilkov, S. Kandhasamy, E. Payne, and R. L. Savage, Fiducial Displacements with Improved Accuracy for the Global Network of Gravitational Wave Detectors, Classical Quantum Gravity 38, 015009 (2021).

[73] D. Estevez, P. Lagabbe, A. Masserot, L. Rolland, M. SeglarArroyo, and D. Verkindt, The Advanced Virgo Photon Calibrators, Classical Quantum Gravity 38, 075007 (2021).

[74] L. Sun et al., Characterization of Systematic Error in Advanced LIGO Calibration, Classical Quantum Gravity 37, 225008 (2020).

[75] B. P. Abbott et al. (LIGO Scientific Collaboration, Virgo Collaboration), GW150914: First Results from the Search for Binary Black Hole Coalescence with Advanced LIGO, Phys. Rev. D 93, 122003 (2016).

[76] S. Vitale, W. Del Pozzo, T. G. F. Li, C. Van Den Broeck, I. Mandel, B. Aylott, and J. Veitch, Effect of Calibration Errors on Bayesian Parameter Estimation for Gravitational Wave Signals from Inspiral Binary Systems in the Advanced Detectors Era, Phys. Rev. D 85, 064034 (2012).

[77] D. Estevez, B. Mours, L. Rolland, and D.Verkindt, Online $h(t)$ Reconstruction for Virgo O3 Data: Start of O3, Technical Report No. VIR-0652B-19, Virgo, 2019, https://tds.ego-gw.it/ql/?c=14486.

[78] D. Davis, T. J. Massinger, A. P. Lundgren, J. C. Driggers, A. L. Urban, and L. K. Nuttall, Improving the Sensitivity of Advanced LIGO Using Noise Subtraction, Classical Quantum Gravity 36, 055011 (2019).

[79] A. Viets and M. Wade, Subtracting Narrow-band Noise from LIGO Strain Data in the Third Observing Run, Technical Report No. T2100058, LIGO DCC, 2021, https://dcc.ligo.org/LIGO-T2100058/public.

[80] G. Vajente, Y. Huang, M. Isi, J. C. Driggers, J. S. Kissel, M. J. Szczepanczyk, and S. Vitale, Machine-Learning Nonstationary Noise out of Gravitational-Wave Detectors, Phys. Rev. D 101, 042003 (2020).

[81] L. Rolland, M. Seglar-Arroyo, and D. Verkindt, Reprocessing of $h(t)$ for the last two weeks of O3a, Technical Report No. VIR-1201A-19, Virgo, 2019.
[82] D. Davis et al., LIGO Detector Characterization in the Second and Third Observing Runs, arXiv:2101.11673.

[83] D. Macleod, A. L. Urban, M. Isi, T. M. Paulaltin, M. Pitkin, and A. Nitz, gwpy/gwsumm: 1.0.2, 2019.

[84] F. Robinet, Omicron: An Algorithm to Detect and Characterize Transient Noise in Gravitational-Wave Detectors, Technical Report No. VIR-0545C-14, Virgo, 2015.

[85] F. Robinet, N. Arnaud, N. Leroy, A. Lundgren, D. Macleod, and J. McIver, Omicron: A Tool to Characterize Transient Noise in Gravitational-Wave Detectors, SoftwareX 12, 100620 (2020).

[86] T. Accadia et al., Noise from Scattered Light in Virgo's Second Science Run Data, Classical Quantum Gravity 27, 194011 (2010).

[87] E. Flanagan and K. Thorne, Noise due to Backscatter Off Baffles, the Nearby Wall, and Objects at the Far End of the Beam Tube, and Recommended Actions, Technical Report No. LIGO-T940063-00-R, LIGO, 1994.

[88] S. Soni et al. (LIGO Collaboration), Reducing Scattered Light in LIGO's Third Observing Run, Classical Quantum Gravity 38, 025016 (2021).

[89] S. Soni et al., Discovering Features in Gravitational-Wave Data through Detector Characterization, Citizen Science and Machine Learning, arXiv:2103.12104.

[90] M. Cabero et al., Blip Glitches in Advanced LIGO Data, Classical Quantum Gravity 36, 155010 (2019).

[91] D. Davis, L. V. White, and P. R. Saulson, Utilizing aLIGO Glitch Classifications to Validate Gravitational-Wave Candidates, Classical Quantum Gravity 37, 145001 (2020).

[92] B. P. Abbott et al. (LIGO Scientific Collaboration, Virgo Collaboration), Effects of Data Quality Vetoes on a Search for Compact Binary Coalescences in Advanced LIGO's First Observing Run, Classical Quantum Gravity 35, 065010 (2018).

[93] P. Godwin et al., Incorporation of Statistical Data Quality Information into the GstLAL Search Analysis, Technical Report No. P2000117, LSC, 2020.

[94] R. Essick, P. Godwin, C. Hanna, L. Blackburn, and E. Katsavounidis, iDQ: Statistical Inference of Non-Gaussian Noise with Auxiliary Degrees of Freedom in Gravitational-Wave Detectors, arXiv:2005.12761.

[95] S. Chatterji, L. Blackburn, G. Martin, and E. Katsavounidis, Multiresolution Techniques for the Detection of Gravitational-Wave Bursts, Classical Quantum Gravity 21, S1809 (2004).

[96] M. Zevin et al., Gravity Spy: Integrating Advanced LIGO Detector Characterization, Machine Learning, and Citizen Science, Classical Quantum Gravity 34, 064003 (2017).

[97] S. Mozzon, L. K. Nuttall, A. Lundgren, T. Dent, S. Kumar, and A.H. Nitz, Dynamic Normalization for Compact Binary Coalescence Searches in Non-Stationary Noise, Classical Quantum Gravity 37, 215014 (2020).

[98] J. S. Areeda, J. R. Smith, A. P. Lundgren, E. Maros, D. M. Macleod, and J. Zweizig, LigoDV-web: Providing Easy, Secure and Universal Access to a Large Distributed Scientific Data Store for the LIGO Scientific Collaboration, Astron. Comput. 18, 27 (2017). 
[99] D. Verkindt, The Virgo Data Display, Technical Report No. VIR-0237A-15, Virgo, 2015, https://tds.ego-gw.it/q1/? $\mathrm{c}=10957$.

[100] D. Verkindt, Advanced Virgo: From Detector Monitoring to Gravitational Wave Alerts, in Proceedings of the 54th Rencontres de Moriond on Gravitation (Moriond Gravitation 2019), La Thuile, Italy, 2019 (ARISF, 2019), p. 57, https://cds.cern.ch/record/2653662/files/

Moriond_Gravitation_2019.pdf?

subformat $=$ pdfa\&version $=1 \#$ page $=65$.

[101] A. Effler, R. M. S. Schofield, V. V. Frolov, G. González, K. Kawabe, J. R. Smith, J. Birch, and R. McCarthy, Environmental Influences on the LIGO Gravitational Wave Detectors during the 6th Science Run, Classical Quantum Gravity 32, 035017 (2015).

[102] P. Nguyen et al., Environmental Noise in Advanced LIGO Detectors, arXiv:2101.09935.

[103] G. Hemming and D. Verkindt, Virgo Interferometer Monitor (VIM) Web User Interface (WUI) User Guide, Technical Report No. VIR-0546A-16, Virgo, 2016, https:// tds.ego-gw.it/q1/?c=11869.

[104] G. Hemming and D. Verkindt, Virgo Interferometer Monitor (VIM) Plot Generator Users Guide, Technical Report No. VIR-0554A-16, Virgo, 2016, https://tds.egogw.it/ql/?c=11877.

[105] J. R. Smith, T. Abbott, E. Hirose, N. Leroy, D. Macleod, J. McIver, P. Saulson, and P. Shawhan, A Hierarchical Method for Vetoing Noise Transients in GravitationalWave Detectors, Classical Quantum Gravity 28, 235005 (2011).

[106] T. Isogai (LIGO Scientific Collaboration, Virgo Collaboration), Used Percentage Veto for LIGO and Virgo Binary Inspiral Searches, J. Phys. Conf. Ser. 243, 012005 (2010).

[107] N. J. Cornish and T. B. Littenberg, Bayeswave: Bayesian Inference for Gravitational Wave Bursts and Instrument Glitches, Classical Quantum Gravity 32, 135012 (2015).

[108] T. B. Littenberg, J. B. Kanner, N. J. Cornish, and M. Millhouse, Enabling High Confidence Detections of Gravitational-Wave Bursts, Phys. Rev. D 94, 044050 (2016).

[109] N. J. Cornish, T. B. Littenberg, B. Bécsy, K. Chatziioannou, J. A. Clark, S. Ghonge, and M. Millhouse, The BayesWave Analysis Pipeline in the Era of Gravitational Wave Observations, Phys. Rev. D 103, 044006 (2021).

[110] C. Pankow et al., Mitigation of the Instrumental Noise Transient in Gravitational-Wave Data Surrounding GW170817, Phys. Rev. D 98, 084016 (2018).

[111] C. Messick et al., Analysis Framework for the Prompt Discovery of Compact Binary Mergers in Gravitationalwave Data, Phys. Rev. D 95, 042001 (2017).

[112] S. Klimenko et al., Method for Detection and Reconstruction of Gravitational Wave Transients with Networks of Advanced Detectors, Phys. Rev. D 93, 042004 (2016).

[113] T. Dal Canton, A. H. Nitz, B. Gadre, G. S. Davies, V. Villa-Ortega, T. Dent, I. Harry, and L. Xiao, Realtime Search for Compact Binary Mergers in Advanced LIGO and Virgo's Third Observing Run Using PyCBC Live, arXiv:2008.07494.
[114] T. Adams, D. Buskulic, V. Germain, G. M. Guidi, F. Marion, M. Montani, B. Mours, F. Piergiovanni, and G. Wang, Low-Latency Analysis Pipeline for Compact Binary Coalescences in the Advanced Gravitational Wave Detector Era, Classical Quantum Gravity 33, 175012 (2016).

[115] Q. Chu, Low-Latency Detection and Localization of Gravitational Waves from Compact Binary Coalescences, Ph.D. thesis, The University of Western Australia, 2017.

[116] B. P. Abbott et al. (LIGO Scientific Collaboration, Virgo Collaboration), Low-Latency Gravitational-Wave Alerts for Multimessenger Astronomy during the Second Advanced LIGO and Virgo Observing Run, Astrophys. J. 875, 161 (2019).

[117] B. J. Owen and B. S. Sathyaprakash, Matched Filtering of Gravitational Waves from Inspiraling Compact Binaries: Computational Cost and Template Placement, Phys. Rev. D 60, 022002 (1999).

[118] S. Klimenko and G. Mitselmakher, A Wavelet Method for Detection of Gravitational Wave Bursts, Classical Quantum Gravity 21, S1819 (2004).

[119] S. Klimenko, S. Mohanty, M. Rakhmanov, and G. Mitselmakher, Constraint Likelihood Analysis for a Network of Gravitational Wave Detectors, Phys. Rev. D 72, 122002 (2005).

[120] S. Klimenko, S. Mohanty, M. Rakhmanov, and G. Mitselmakher, Constraint Likelihood Method: Generalization for Colored Noise, J. Phys. Conf. Ser. 32, 12 (2006).

[121] S. Klimenko, G. Vedovato, M. Drago, G. Mazzolo, G. Mitselmakher, C. Pankow, G. Prodi, V. Re, F. Salemi, and I. Yakushin, Localization of Gravitational Wave Sources with Networks of Advanced Detectors, Phys. Rev. D 83, 102001 (2011).

[122] S. Sachdev et al., The GstLAL Search Analysis Methods for Compact Binary Mergers in Advanced LIGO's Second and Advanced Virgo's First Observing Runs, arXiv:1901.08580.

[123] C. Hanna, S. Caudill, C. Messick, A. Reza, S. Sachdev, L. Tsukada, K. Cannon, K. Blackburn, J. D. E. Creighton, H. Fong, P. Godwin, S. Kapadia, T. G. F. Li, R. Magee, D. Meacher, D. Mukherjee, A. Pace, S. Privitera, R. K. L. Lo, and L. Wade, Fast Evaluation of Multidetector Consistency for Real-Time Gravitational Wave Searches, Phys. Rev. D 101, 022003 (2020).

[124] B. Allen, $\chi^{2}$ Time-Frequency Discriminator for Gravitational Wave Detection, Phys. Rev. D 71, 062001 (2005).

[125] T. Dal Canton et al., Implementing a Search for AlignedSpin Neutron Star-Black Hole Systems with Advanced Ground-Based Gravitational Wave Detectors, Phys. Rev. D 90, 082004 (2014).

[126] S. A. Usman et al., The PyCBC Search for Gravitational Waves from Compact Binary Coalescence, Classical Quantum Gravity 33, 215004 (2016).

[127] A. H. Nitz, T. Dent, T. Dal Canton, S. Fairhurst, and D. A. Brown, Detecting Binary Compact-Object Mergers with Gravitational Waves: Understanding and Improving the Sensitivity of the PуСвC Search, Astrophys. J. 849, 118 (2017). 
[128] F. Aubin et al., The MBTA Pipeline for Detecting Compact Binary Coalescences in the Third LIGO-Virgo Observing Run, Classical Quantum Gravity 38, 095004 (2021).

[129] B. P. Abbott et al. (LIGO Scientific Collaboration, Virgo Collaboration), All-Sky Search for Short GravitationalWave Bursts in the Second Advanced LIGO and Advanced Virgo Run, Phys. Rev. D 100, 024017 (2019).

[130] B. P. Abbott et al. (LIGO Scientific Collaboration, Virgo Collaboration), Observing Gravitational-Wave Transient GW150914 with Minimal Assumptions, Phys. Rev. D 93, 122004 (2016); Addendum Phys. Rev. D 94, 069903 (2016).

[131] V. Necula, S. Klimenko, and G. Mitselmakher, Transient Analysis with Fast Wilson-Daubechies Time-Frequency Transform, J. Phys. Conf. Ser. 363, 012032 (2012).

[132] J. Aasi et al. (Virgo Collaboration), The Characterization of Virgo Data and Its Impact on Gravitational-Wave Searches, Classical Quantum Gravity 29, 155002 (2012).

[133] V. Tiwari, S. Klimenko, V. Necula, and G. Mitselmakher, Reconstruction of Chirp Mass in Searches for Gravitational Wave Transients, Classical Quantum Gravity 33, 01LT01 (2016).

[134] M. Szczepanczyk et al., Observing an Intermediate-Mass Black Hole GW190521 with Minimal Assumptions, arXiv: 2009.11336 [Phys. Rev. D (to be published)].

[135] M. Was, M.-A. Bizouard, V. Brisson, F. Cavalier, M. Davier, P. Hello, N. Leroy, F. Robinet, and M. Vavoulidis, On the Background Estimation by Time Slides in a Network of Gravitational Wave Detectors, Classical Quantum Gravity 27, 015005 (2010).

[136] J. C. Bustillo, F. Salemi, T. Dal Canton, and K. P. Jani, Sensitivity of Gravitational Wave Searches to the Full Signal of Intermediate-Mass Black Hole Binaries during the First Observing Run of Advanced LIGO, Phys. Rev. D 97, 024016 (2018).

[137] B. Abbott et al. (LIGO Scientific Collaboration, Virgo Collaboration), Search for Eccentric Binary Black Hole Mergers with Advanced LIGO and Advanced Virgo during Their First and Second Observing Runs, Astrophys. J. 883, 149 (2019).

[138] A. Nitz et al., gwastro/pycbc: PyCBC release v1.15.0, 2019.

[139] L. Blanchet, T. Damour, B. R. Iyer, C. M. Will, and A. G. Wiseman, Gravitational Radiation Damping of Compact Binary Systems to Second Post-Newtonian Order, Phys. Rev. Lett. 74, 3515 (1995).

[140] P. Ajith et al., Inspiral-Merger-Ringdown Waveforms for Black-Hole Binaries with Nonprecessing Spins, Phys. Rev. Lett. 106, 241101 (2011).

[141] P. C. Peters, Gravitational Radiation and the Motion of Two Point Masses, Phys. Rev. 136, B1224 (1964).

[142] A. Krolak and B. F. Schutz, Coalescing Binaries-Probe of the Universe, Gen. Relativ. Gravit. 19, 1163 (1987).

[143] C. Cutler and E. E. Flanagan, Gravitational Waves from Merging Compact Binaries: How Accurately Can One Extract the Binary's Parameters from the Inspiral Wave Form?, Phys. Rev. D 49, 2658 (1994).

[144] S. Privitera, S. R. P. Mohapatra, P. Ajith, K. Cannon, N. Fotopoulos, M. A. Frei, C. Hanna, A. J. Weinstein, and J. T. Whelan, Improving the Sensitivity of a Search for Coalescing Binary Black Holes with Nonprecessing Spins in Gravitational Wave Data, Phys. Rev. D 89, 024003 (2014).

[145] I. W. Harry, B. Allen, and B. S. Sathyaprakash, A Stochastic Template Placement Algorithm for Gravitational Wave Data Analysis, Phys. Rev. D 80, 104014 (2009).

[146] D. Mukherjee et al., The GstLaL Template Bank for Spinning Compact Binary Mergers in the Second Observation Run of Advanced LIGO and Virgo, arXiv:1812 .05121 [Phys. Rev. D (to be published)].

[147] B. S. Sathyaprakash and S. V. Dhurandhar, Choice of Filters for the Detection of Gravitational Waves from Coalescing Binaries, Phys. Rev. D 44, 3819 (1991).

[148] E. Poisson, Gravitational Waves from Inspiraling Compact Binaries: The Quadrupole Moment Term, Phys. Rev. D 57, 5287 (1998).

[149] T. Damour, P. Jaranowski, and G. Schaefer, Dimensional Regularization of the Gravitational Interaction of Point Masses, Phys. Lett. B 513, 147 (2001).

[150] B. Mikóczi, M. Vasuth, and L. A. Gergely, Self-Interaction Spin Effects in Inspiralling Compact Binaries, Phys. Rev. D 71, 124043 (2005).

[151] L. Blanchet, T. Damour, G. Esposito-Farese, and B. R. Iyer, Dimensional Regularization of the Third PostNewtonian Gravitational Wave Generation from Two Point Masses, Phys. Rev. D 71, 124004 (2005).

[152] K. G. Arun, A. Buonanno, G. Faye, and E. Ochsner, Higher-Order Spin Effects in the Amplitude and Phase of Gravitational Waveforms Emitted by Inspiraling Compact Binaries: Ready-to-Use Gravitational Waveforms, Phys. Rev. D 79, 104023 (2009); Erratum, Phys. Rev. D 84, 049901 (2011).

[153] A. Buonanno, B. R. Iyer, E. Ochsner, Y. Pan, and B. S. Sathyaprakash, Comparison of Post-Newtonian Templates for Compact Binary Inspiral Signals in GravitationalWave Detectors, Phys. Rev. D 80, 084043 (2009).

[154] A. Bohé, S. Marsat, and L. Blanchet, Next-to-Next-toLeading Order Spin-Orbit Effects in the Gravitational Wave Flux and Orbital Phasing of Compact Binaries, Classical Quantum Gravity 30, 135009 (2013).

[155] A. Bohé, G. Faye, S. Marsat, and E. K. Porter, Quadraticin-Spin Effects in the Orbital Dynamics and GravitationalWave Energy Flux of Compact Binaries at the 3PN Order, Classical Quantum Gravity 32, 195010 (2015).

[156] C. K. Mishra, A. Kela, K. G. Arun, and G. Faye, Ready-toUse Post-Newtonian Gravitational Waveforms for Binary Black Holes with Nonprecessing Spins: An Update, Phys. Rev. D 93, 084054 (2016).

[157] A. Bohé, L. Shao, A. Taracchini, A. Buonanno, S. Babak, I. W. Harry, I. Hinder, S. Ossokine, M. Pürrer, V. Raymond, T. Chu, H. Fong, P. Kumar, H. P. Pfeiffer, M. Boyle, D. A. Hemberger, L. E. Kidder, G. Lovelace, M. A. Scheel, and B. Szilágyi, Improved Effective-One-Body Model of Spinning, Nonprecessing Binary Black Holes for the Era of Gravitational-Wave Astrophysics with Advanced Detectors, Phys. Rev. D 95, 044028 (2017).

[158] T. Dal Canton and I. W. Harry, Designing a Template Bank to Observe Compact Binary Coalescences in Advanced LIGO's Second Observing Run, arXiv:1705.01845.

[159] S. Roy, A. S. Sengupta, and N. Thakor, Hybrid GeometricRandom Template-Placement Algorithm for Gravitational 
Wave Searches from Compact Binary Coalescences, Phys. Rev. D 95, 104045 (2017).

[160] S. Roy, A. S. Sengupta, and P. Ajith, Effectual Template Banks for Upcoming Compact Binary Searches in Advanced-LIGO and Virgo Data, Phys. Rev. D 99, 024048 (2019).

[161] K. Cannon, C. Hanna, and J. Peoples, Likelihood-Ratio Ranking Statistic for Compact Binary Coalescence Candidates with Rate Estimation, arXiv:1504.04632.

[162] K. Cannon, C. Hanna, and D. Keppel, Method to Estimate the Significance of Coincident Gravitational-Wave Observations from Compact Binary Coalescence, Phys. Rev. D 88, 024025 (2013).

[163] H. K. Y. Fong, From Simulations to Signals: Analyzing Gravitational Waves from Compact Binary Coalescences, Ph.D. thesis, Toronto University, 2018.

[164] T. Dent and J. Veitch, Optimizing Gravitational-Wave Searches for a Population of Coalescing Binaries: Intrinsic Parameters, Phys. Rev. D 89, 062002 (2014).

[165] S. Babak et al., Searching for Gravitational Waves from Binary Coalescence, Phys. Rev. D 87, 024033 (2013).

[166] G. S. Davies, T. Dent, M. Tápai, I. Harry, C. McIsaac, and A. H. Nitz, Extending the PyCBC Search for Gravitational Waves from Compact Binary Mergers to a Global Network, Phys. Rev. D 102, 022004 (2020).

[167] B. Zackay, T. Venumadhav, J. Roulet, L. Dai, and M. Zaldarriaga, Detecting Gravitational Waves in Data with Non-Gaussian Noise, arXiv:1908.05644.

[168] B. P. Abbott et al. (LIGO Scientific Collaboration, Virgo Collaboration), The Rate of Binary Black Hole Mergers Inferred from Advanced LIGO Observations Surrounding GW150914, Astrophys. J. 833, L1 (2016).

[169] The measure $\mathcal{V}$ is not equivalent to a geometric VT, because both the injected population density and the comoving volume element vary over redshift.

[170] W. M. Farr, J. R. Gair, I. Mandel, and C. Cutler, Counting And Confusion: Bayesian Rate Estimation With Multiple Populations, Phys. Rev. D 91, 023005 (2015).

[171] B. P. Abbott et al. (LIGO Scientific Collaboration, Virgo Collaboration), Supplement: The Rate of Binary Black Hole Mergers Inferred from Advanced LIGO Observations Surrounding GW150914, Astrophys. J. Suppl. Ser. 227, 14 (2016).

[172] LIGO Scientific Collaboration and Virgo Collaboration, GCN 24168, 2019, https://gcn.gsfc.nasa.gov/gcn3/24168 .gcn 3 .

[173] There is some ambiguity regarding the $\mathrm{BBH}$ nature of GW190814 [33]. Nevertheless, its chirp mass and total mass are not inconsistent with those of BBHs.

[174] LIGO Scientific Collaboration and Virgo Collaboration, GCN 24237, 2019, https://gcn.gsfc.nasa.gov/gcn3/24237 .gcn 3 .

[175] J. Creighton, Certain Identities in FGMC, Technical Report No. T1700029-v2, LIGO DCC, 2017, https://dcc .ligo.org/LIGO-T1700029/public.

[176] S. J. Kapadia et al., A Self-Consistent Method to Estimate the Rate of Compact Binary Coalescences with a Poisson Mixture Model, Classical Quantum Gravity 37, 045007 (2020).
[177] B. P. Abbott et al. (LIGO Scientific Collaboration, Virgo Collaboration), Properties of the Binary Black Hole Merger GW150914, Phys. Rev. Lett. 116, 241102 (2016).

[178] C. P. Berry et al., Parameter Estimation for Binary Neutron-Star Coalescences with Realistic Noise during the Advanced LIGO Era, Astrophys. J. 804, 114 (2015).

[179] J. Veitch et al., Parameter Estimation for Compact Binaries with Ground-Based Gravitational-Wave Observations Using the LALInference Software Library, Phys. Rev. D 91, 042003 (2015).

[180] L.S. Finn, Detection, Measurement and Gravitational Radiation, Phys. Rev. D 46, 5236 (1992).

[181] T. B. Littenberg and N. J. Cornish, Bayesian Inference for Spectral Estimation of Gravitational Wave Detector Noise, Phys. Rev. D 91, 084034 (2015).

[182] K. Chatziioannou, C.-J. Haster, T. B. Littenberg, W. M. Farr, S. Ghonge, M. Millhouse, J. A. Clark, and N. Cornish, Noise Spectral Estimation Methods and Their Impact on Gravitational Wave Measurement of Compact Binary Mergers, Phys. Rev. D 100, 104004 (2019).

[183] F. Hofmann, E. Barausse, and L. Rezzolla, The Final Spin from Binary Black Holes in Quasi-circular Orbits, Astrophys. J. Lett. 825, L19 (2016).

[184] X. Jiménez-Forteza, D. Keitel, S. Husa, M. Hannam, S. Khan, and M. Pürrer, Hierarchical Data-Driven Approach to Fitting Numerical Relativity Data for Nonprecessing Binary Black Holes with an Application to Final Spin and Radiated Energy, Phys. Rev. D 95, 064024 (2017).

[185] J. Healy and C. O. Lousto, Remnant of Binary Black-Hole Mergers: New Simulations and Peak Luminosity Studies, Phys. Rev. D 95, 024037 (2017).

[186] N. K. Johnson-McDaniel, A. Gupta, P. Ajith, D. Keitel, O. Birnholtz, F. Ohme, and S. Husa, Determining the Final Spin of a Binary Black Hole System including In-Plane Spins: Method and Checks of Accuracy, Technical Report No. T1600168, LSC, 2016, https://dcc.ligo.org/LIGOT1600168/public.

[187] D. Keitel et al., The Most Powerful Astrophysical Events: Gravitational-Wave Peak Luminosity of Binary Black Holes as Predicted by Numerical Relativity, Phys. Rev. D 96, 024006 (2017).

[188] K. Yagi and N. Yunes, Approximate Universal Relations for Neutron Stars and Quark Stars, Phys. Rep. 681, 1 (2017).

[189] M. Favata, Systematic Parameter Errors in Inspiraling Neutron Star Binaries, Phys. Rev. Lett. 112, 101101 (2014).

[190] L. Wade, J. D. E. Creighton, E. Ochsner, B. D. Lackey, B. F. Farr, T. B. Littenberg, and V. Raymond, Systematic and Statistical Errors in a Bayesian Approach to the Estimation of the Neutron-Star Equation of State Using Advanced Gravitational Wave Detectors, Phys. Rev. D 89, 103012 (2014).

[191] T. Binnington and E. Poisson, Relativistic Theory of Tidal Love Numbers, Phys. Rev. D 80, 084018 (2009).

[192] T. Damour and A. Nagar, Relativistic Tidal Properties of Neutron Stars, Phys. Rev. D 80, 084035 (2009).

[193] P. Landry and E. Poisson, Tidal Deformation of a Slowly Rotating Material Body. External Metric, Phys. Rev. D 91, 104018 (2015). 
[194] P. Pani, L. Gualtieri, A. Maselli, and V. Ferrari, Tidal Deformations of a Spinning Compact Object, Phys. Rev. D 92, 024010 (2015).

[195] S. Husa, S. Khan, M. Hannam, M. Pürrer, F. Ohme, X. J. Forteza, and A. Bohé, Frequency-Domain Gravitational Waves from Nonprecessing Black-Hole Binaries. I. New Numerical Waveforms and Anatomy of the Signal, Phys. Rev. D 93, 044006 (2016).

[196] S. Khan, S. Husa, M. Hannam, F. Ohme, M. Pürrer, X. J. Forteza, and A. Bohé, Frequency-Domain Gravitational Waves from Nonprecessing Black-Hole Binaries. II. A Phenomenological Model for the Advanced Detector Era, Phys. Rev. D 93, 044007 (2016).

[197] M. Hannam, P. Schmidt, A. Bohé, L. Haegel, S. Husa, F. Ohme, G. Pratten, and M. Pürrer, Simple Model of Complete Precessing Black-Hole-Binary Gravitational Waveforms, Phys. Rev. Lett. 113, 151101 (2014).

[198] P. Schmidt, F. Ohme, and M. Hannam, Towards Models of Gravitational Waveforms from Generic Binaries II: Modelling Precession Effects with a Single Effective Precession Parameter, Phys. Rev. D 91, 024043 (2015).

[199] S. Ossokine et al., Multipolar Effective-One-Body Waveforms for Precessing Binary Black Holes: Construction and Validation, Phys. Rev. D 102, 044055 (2020).

[200] A. Buonanno and T. Damour, Effective One-Body Approach to General Relativistic Two-Body Dynamics, Phys. Rev. D 59, 084006 (1999).

[201] A. Buonanno and T. Damour, Transition from Inspiral to Plunge in Binary Black Hole Coalescences, Phys. Rev. D 62, 064015 (2000).

[202] P. Schmidt, M. Hannam, S. Husa, and P. Ajith, Tracking the Precession of Compact Binaries from Their Gravitational-Wave Signal, Phys. Rev. D 84, 024046 (2011).

[203] M. Boyle, R. Owen, and H. P. Pfeiffer, A Geometric Approach to the Precession of Compact Binaries, Phys. Rev. D 84, 124011 (2011).

[204] R. O’Shaughnessy, B. Vaishnav, J. Healy, Z. Meeks, and D. Shoemaker, Efficient Asymptotic Frame Selection for Binary Black Hole Spacetimes Using Asymptotic Radiation, Phys. Rev. D 84, 124002 (2011).

[205] E. Ochsner and R. O'Shaughnessy, Asymptotic Frame Selection for Binary Black Hole Spacetimes II: PostNewtonian Limit, Phys. Rev. D 86, 104037 (2012).

[206] P. Schmidt, M. Hannam, and S. Husa, Towards Models of Gravitational Waveforms from Generic Binaries: A Simple Approximate Mapping between Precessing and Non-precessing Inspiral Signals, Phys. Rev. D 86, 104063 (2012).

[207] S. Khan, K. Chatziioannou, M. Hannam, and F. Ohme, Phenomenological Model for the Gravitational-Wave Signal from Precessing Binary Black Holes with TwoSpin Effects, Phys. Rev. D 100, 024059 (2019).

[208] S. Khan, F. Ohme, K. Chatziioannou, and M. Hannam, Including Higher Order Multipoles in Gravitational-Wave Models for Precessing Binary Black Holes, Phys. Rev. D 101, 024056 (2020).

[209] S. Babak, A. Taracchini, and A. Buonanno, Validating the Effective-One-Body Model of Spinning, Precessing Binary Black Holes against Numerical Relativity, Phys. Rev. D 95, 024010 (2017).
[210] V. Varma, S. E. Field, M. A. Scheel, J. Blackman, D. Gerosa, L. C. Stein, L. E. Kidder, and H. P. Pfeiffer, Surrogate Models for Precessing Binary Black Hole Simulations with Unequal Masses, Phys. Rev. Research 1, 033015 (2019).

[211] L. London, S. Khan, E. Fauchon-Jones, C. García, M. Hannam, S. Husa, X. Jiménez-Forteza, C. Kalaghatgi, F. Ohme, and F. Pannarale, First Higher-Multipole Model of Gravitational Waves from Spinning and Coalescing BlackHole Binaries, Phys. Rev. Lett. 120, 161102 (2018).

[212] R. Cotesta, A. Buonanno, A. Bohé, A. Taracchini, I. Hinder, and S. Ossokine, Enriching the Symphony of Gravitational Waves from Binary Black Holes by Tuning Higher Harmonics, Phys. Rev. D 98, 084028 (2018).

[213] T. Dietrich, S. Khan, R. Dudi, S. J. Kapadia, P. Kumar, A. Nagar, F. Ohme, F. Pannarale, A. Samajdar, S. Bernuzzi, G. Carullo, W. Del Pozzo, M. Haney, C. Markakis, M. Pürrer, G. Riemenschneider, Y. E. Setyawati, K. W. Tsang, and C. Van Den Broeck, Matter Imprints in Waveform Models for Neutron Star Binaries: Tidal and Self-Spin Effects, Phys. Rev. D 99, 024029 (2019).

[214] T. Dietrich, S. Bernuzzi, and W. Tichy, Closed-Form Tidal Approximants for Binary Neutron Star Gravitational Waveforms Constructed from High-Resolution Numerical Relativity Simulations, Phys. Rev. D 96, 121501(R) (2017).

[215] T. Damour, A. Nagar, and L. Villain, Measurability of the Tidal Polarizability of Neutron Stars in Late-Inspiral Gravitational-Wave Signals, Phys. Rev. D 85, 123007 (2012).

[216] A. Maselli, V. Cardoso, V. Ferrari, L. Gualtieri, and P. Pani, Equation-of-State-Independent Relations in Neutron Stars, Phys. Rev. D 88, 023007 (2013).

[217] S. Bernuzzi, A. Nagar, T. Dietrich, and T. Damour, Modeling the Dynamics of Tidally Interacting Binary Neutron Stars up to the Merger, Phys. Rev. Lett. 114, 161103 (2015).

[218] B. D. Lackey, M. Pürrer, A. Taracchini, and S. Marsat, Surrogate Model for an Aligned-Spin Effective One Body Waveform Model of Binary Neutron Star Inspirals Using Gaussian Process Regression, Phys. Rev. D 100, 024002 (2019).

[219] T. Hinderer et al., Effects of Neutron-Star Dynamic Tides on Gravitational Waveforms within the Effective-OneBody Approach, Phys. Rev. Lett. 116, 181101 (2016).

[220] J. Steinhoff, T. Hinderer, A. Buonanno, and A. Taracchini, Dynamical Tides in General Relativity: Effective Action and Effective-One-Body Hamiltonian, Phys. Rev. D 94, 104028 (2016).

[221] A. Matas et al., Aligned-Spin Neutron-Star-Black-Hole Waveform Model Based on the Effective-One-Body Approach and Numerical-Relativity Simulations, Phys. Rev. D 102, 043023 (2020).

[222] J. E. Thompson, E. Fauchon-Jones, S. Khan, E. Nitoglia, F. Pannarale, T. Dietrich, and M. Hannam, Modeling the Gravitational Wave Signature of Neutron Star Black Hole Coalescences: PhenomNSBH, Phys. Rev. D 101, 124059 (2020).

[223] L. Santamaria et al., Matching Post-Newtonian and Numerical Relativity Waveforms: Systematic Errors and 
a New Phenomenological Model for Non-precessing Black Hole Binaries, Phys. Rev. D 82, 064016 (2010).

[224] T. Dietrich, A. Samajdar, S. Khan, N. K. JohnsonMcDaniel, R. Dudi, and W. Tichy, Improving the NRTidal Model for Binary Neutron Star Systems, Phys. Rev. D 100, 044003 (2019).

[225] G. Ashton and S. Khan, Multiwaveform Inference of Gravitational Waves, Phys. Rev. D 101, 064037 (2020).

[226] R. Cotesta, S. Marsat, and M. Pürrer, Frequency Domain Reduced Order Model of Aligned-Spin Effective-One-Body Waveforms with Higher-Order Modes, Phys. Rev. D 101, 124040 (2020).

[227] Y. Pan, A. Buonanno, A. Taracchini, L. E. Kidder, A. H. Mroué, H. P. Pfeiffer, M. A. Scheel, and B. Szilágyi, Inspiral-Merger-Ringdown Waveforms of Spinning, Precessing Black-Hole Binaries in the Effective-One-Body Formalism, Phys. Rev. D 89, 084006 (2014).

[228] J. Skilling, Nested Sampling for General Bayesian Computation, Bayesian Anal. 1, 833 (2006).

[229] C. Pankow, P. Brady, E. Ochsner, and R. O'Shaughnessy, Novel Scheme for Rapid Parallel Parameter Estimation of Gravitational Waves from Compact Binary Coalescences, Phys. Rev. D 92, 023002 (2015).

[230] J. Lange et al., Parameter Estimation Method That Directly Compares Gravitational Wave Observations to Numerical Relativity, Phys. Rev. D 96, 104041 (2017).

[231] D. Wysocki, R. O'Shaughnessy, J. Lange, and Y.-L. L. Fang, Accelerating Parameter Inference with Graphics Processing Units, Phys. Rev. D 99, 084026 (2019).

[232] R. J. E. Smith, G. Ashton, A. Vajpeyi, and C. Talbot, Massively Parallel Bayesian Inference for Transient Gravitational-Wave Astronomy, Mon. Not. R. Astron. Soc. 498, 4492 (2020).

[233] G. Ashton et al., BILBY: A User-Friendly Bayesian Inference Library for Gravitational-Wave Astronomy, Astrophys. J. Suppl. Ser. 241, 27 (2019).

[234] I. M. Romero-Shaw et al., Bayesian Inference for Compact Binary Coalescences with Bilby: Validation and Application to the First LIGO-Virgo Gravitational-Wave Transient Catalogue, Mon. Not. R. Astron. Soc. 499, 3295 (2020).

[235] J. S. Speagle, DYNESTY: A Dynamic Nested Sampling Package for Estimating Bayesian Posteriors and Evidences, Mon. Not. R. Astron. Soc. 493, 3132 (2020).

[236] R. Abbott et al. (LIGO Scientific Collaboration, Virgo Collaboration), GW190412: Observation of a BinaryBlack-Hole Coalescence with Asymmetric Masses, Phys. Rev. D 102, 043015 (2020).

[237] C. Hoy and V. Raymond, PESummary: The Code Agnostic Parameter Estimation Summary Page Builder, arXiv: 2006.06639.

[238] P. Ade et al. (Planck Collaboration), Planck 2015 Results. XIII. Cosmological Parameters, Astron. Astrophys. 594, A13 (2016).

[239] https://emfollow.docs.ligo.org/userguide/analysis/index .html\#alert-threshold.

[240] Had we only applied a $2.0 \mathrm{yr}^{-1}$ FAR threshold in GWTC-1 and not a $p_{\text {astro }}$ threshold, one additional marginal candidate event from GWTC-1 would have passed the criteria we use here: 170616.
[241] LIGO Scientific Collaboration and Virgo Collaboration, GCN 24442, 2019, https://gcn.gsfc.nasa.gov/gen3/24442 .gcn3.

[242] LIGO Scientific Collaboration and Virgo Collaboration, GCN 25087, 2019, https://gcn.gsfc.nasa.gov/gcn3/25087 .gcn3.

[243] LIGO Scientific Collaboration and Virgo Collaboration, GCN 25606, 2019, https://gcn.gsfc.nasa.gov/gcn3/25606 .gcn 3 .

[244] LIGO Scientific Collaboration and Virgo Collaboration, GCN 25695, 2019, https://gcn.gsfc.nasa.gov/gcn3/25695 .gcn 3 .

[245] LIGO Scientific Collaboration and Virgo Collaboration, GCN 25707, 2019, https://gcn.gsfc.nasa.gov/gcn3/25707 .gcn3.

[246] LIGO Scientific Collaboration and Virgo Collaboration, GCN 25814, 2019, https://gcn.gsfc.nasa.gov/gcn3/25814 . $\mathrm{gcn} 3$.

[247] LIGO Scientific Collaboration and Virgo Collaboration, GCN 25876, 2019, https://gen.gsfc.nasa.gov/gen3/25876 .gcn3.

[248] LIGO Scientific Collaboration and Virgo Collaboration, GCN 24045, 2019, https://gcn.gsfc.nasa.gov/gcn3/24045 . $\mathrm{gcn} 3$.

[249] https://gracedb.ligo.org/superevents/public/O3/.

[250] LIGO Scientific Collaboration and Virgo Collaboration, GCN G298048, 2017, https://gcn.gsfc.nasa.gov/other/ G298048.gcn3.

[251] LIGO Scientific Collaboration and Virgo Collaboration, GCN 24462, 2019, https://gcn.gsfc.nasa.gov/gen3/24462 .gcn3.

[252] LIGO Scientific Collaboration and Virgo Collaboration, GCN 24489, 2019, https://gcn.gsfc.nasa.gov/gcn3/24489 .gcn 3 .

[253] LIGO Scientific Collaboration and Virgo Collaboration, GCN 24109, 2019, https://gcn.gsfc.nasa.gov/gcn3/24109 .gcn3.

[254] LIGO Scientific Collaboration and Virgo Collaboration, GCN 24591, 2019, https://gcn.gsfc.nasa.gov/gen3/24591 .gcn3.

[255] LIGO Scientific Collaboration and Virgo Collaboration, GCN 24656. 2019, https://gcn.gsfc.nasa.gov/gcn3/24656 .gcn3.

[256] LIGO Scientific Collaboration and Virgo Collaboration, GCN 25301, 2019, https://gcn.gsfc.nasa.gov/gcn3/25301 .gcn 3 .

[257] LIGO Scientific Collaboration and Virgo Collaboration, GCN 25367, 2019, https://gcn.gsfc.nasa.gov/gen3/25367 .gcn 3 .

[258] LIGO Scientific Collaboration and Virgo Collaboration, GCN 25442, 2019, https://gcn.gsfc.nasa.gov/gcn3/25442 .gcn3.

[259] LIGO Scientific Collaboration and Virgo Collaboration, GCN 25554, 2019, https://gcn.gsfc.nasa.gov/gcn3/25554 . $\mathrm{gcn} 3$.

[260] LIGO Scientific Collaboration and Virgo Collaboration, GCN 25883, 2019, https://gcn.gsfc.nasa.gov/gen3/25883 .gcn3.

[261] Where posterior samples from existing publications are used, we still impose the uniform-in-comoving volume 
prior described in Sec. V C for consistency with the new results in this publication.

[262] LIGO Scientific Collaboration and Virgo Collaboration, GWTC-2 Data Release, https://gw-openscience.org/ GWTC-2/.

[263] C. D. Bailyn, R. K. Jain, P. Coppi, and J. A. Orosz, The Mass Distribution of Stellar Black Holes, Astrophys. J. 499, 367 (1998).

[264] F. Ozel, D. Psaltis, R. Narayan, and J. E. McClintock, The Black Hole Mass Distribution in the Galaxy, Astrophys. J. 725, 1918 (2010).

[265] W. M. Farr, N. Sravan, A. Cantrell, L. Kreidberg, C. D. Bailyn, I. Mandel, and V. Kalogera, The Mass Distribution of Stellar-Mass Black Holes, Astrophys. J. 741, 103 (2011).

[266] F. Ozel, D. Psaltis, R. Narayan, and A. S. Villarreal, On the Mass Distribution and Birth Masses of Neutron Stars, Astrophys. J. 757, 55 (2012).

[267] M. C. Miller and J. M. Miller, The Masses and Spins of Neutron Stars and Stellar-Mass Black Holes, Phys. Rep. 548, 1 (2015).

[268] M. Heida, P. Jonker, M. Torres, and A. Chiavassa, The Mass Function of GX 339-4 from Spectroscopic Observations of Its Donor Star, Astrophys. J. 846, 132 (2017).

[269] T. A. Thompson et al., A Noninteracting Low-Mass Black Hole-Giant Star Binary System, Science 366, 637 (2019).

[270] J. M. Lattimer, The Nuclear Equation of State and Neutron Star Masses, Annu. Rev. Nucl. Part. Sci. 62, 485 (2012).

[271] F. Özel and P. Freire, Masses, Radii, and the Equation of State of Neutron Stars, Annu. Rev. Astron. Astrophys. 54, 401 (2016).

[272] J. Alsing, H. O. Silva, and E. Berti, Evidence for a Maximum Mass Cut-off in the Neutron Star Mass Distribution and Constraints on the Equation of State, Mon. Not. R. Astron. Soc. 478, 1377 (2018).

[273] K. Chatziioannou and W. M. Farr, Inferring the Maximum and Minimum Mass of Merging Neutron Stars with Gravitational Waves, Phys. Rev. D 102, 064063 (2020).

[274] T. M. Tauris et al., Formation of Double Neutron Star Systems, Astrophys. J. 846, 170 (2017).

[275] N. Farrow, X.-J. Zhu, and E. Thrane, The Mass Distribution of Galactic Double Neutron Stars, Astrophys. J. 876, 18 (2019).

[276] M. Safarzadeh, E. Ramirez-Ruiz, and E. Berger, Does GW190425 Require an Alternative Formation Pathway than a Fast-Merging Channel?, Astrophys. J. 900, 13 (2020).

[277] A. Gupta, D. Gerosa, K. G. Arun, E. Berti, W. M. Farr, and B. S. Sathyaprakash, Black Holes in the Low Mass Gap: Implications for Gravitational Wave Observations, Phys. Rev. D 101, 103036 (2020).

[278] I. M. Romero-Shaw, N. Farrow, S. Stevenson, E. Thrane, and X.-J. Zhu, On the Origin of GW190425, Mon. Not. R. Astron. Soc. 496, L64 (2020).

[279] I. Mandel, B. Müller, J. Riley, S. E. de Mink, A. VignaGómez, and D. Chattopadhyay, Binary Population Synthesis with Probabilistic Remnant Mass and Kick Prescriptions, Mon. Not. R. Astron. Soc. 500, 1380 (2020).

[280] J. Lin, Divergence Measures Based on the Shannon Entropy, IEEE Trans. Inf. Theory 37, 145 (1991).
[281] M. Zevin, C. P. L. Berry, S. Coughlin, K. Chatziioannou, and S. Vitale, You Can't Always Get What You Want: The Impact of Prior Assumptions on Interpreting GW190412, Astrophys. J. Lett. 899, L17 (2020).

[282] S. Fairhurst, R. Green, C. Hoy, M. Hannam, and A. Muir, Two-Harmonic Approximation for Gravitational Waveforms from Precessing Binaries, Phys. Rev. D 102, 024055 (2020).

[283] S. Fairhurst, R. Green, M. Hannam, and C. Hoy, When Will We Observe Binary Black Holes Precessing?, Phys. Rev. D 102, 041302(R) (2020).

[284] R. Green, C. Hoy, S. Fairhurst, M. Hannam, F. Pannarale, and C. Thomas, Identifying when Precession Can Be Measured in Gravitational Waveforms, arXiv:2010.04131.

[285] G. Pratten, P. Schmidt, R. Buscicchio, and L. M. Thomas, Measuring Precession in Asymmetric Compact Binaries, Phys. Rev. Research 2, 043096 (2020).

[286] N. Christensen and R. Meyer, Markov Chain Monte Carlo Methods for Bayesian Gravitational Radiation Data Analysis, Phys. Rev. D 58, 082001 (1998).

[287] F. Salemi, E. Milotti, G. A. Prodi, G. Vedovato, C. Lazzaro, S. Tiwari, S. Vinciguerra, M. Drago, and S. Klimenko, Wider Look at the Gravitational-Wave Transients from GWTC-1 Using an Unmodeled Reconstruction Method, Phys. Rev. D 100, 042003 (2019).

[288] S. Ghonge, K. Chatziioannou, J. A. Clark, T. Littenberg, M. Millhouse, L. Cadonati, and N. Cornish, Reconstructing Gravitational Wave Signals from Binary Black Hole Mergers with Minimal Assumptions, Phys. Rev. D 102, 064056 (2020).

[289] T. Akutsu et al. (KAGRA Collaboration), KAGRA: 2.5 Generation Interferometric Gravitational Wave Detector, Nat. Astron. 3, 35 (2019).

[290] B. P. Abbott et al. (KAGRA Collaboration, LIGO Scientific Collaboration, Virgo Collaboration), Prospects for Observing and Localizing Gravitational-Wave Transients with Advanced LIGO, Advanced Virgo and KAGRA, Living Rev. Relativity 23, 3 (2020).

[291] C. Pankow, M. Rizzo, K. Rao, C. P. L. Berry, and V. Kalogera, Localization of Compact Binary Sources with Second Generation Gravitational-wave Interferometer Networks, Astrophys. J. 902, 71 (2020).

[292] D. Macleod, GWOSC Client, https://zenodo.org/record/ 2590960.

[293] LIGO Scientific Collaboration and Virgo Collaboration, GWTC-2 Data Release, https:/gw-openscience.org/ GWTC-2/.

[294] LIGO Scientific Collaboration and Virgo Collaboration, GWTC-2 Data Release: Sensitivity of Matched Filter Searches to Binary Black Hole Merger Populations, https://dcc.ligo.org/P2000217/public.

[295] LIGO Scientific Collaboration, LIGO Algorithm Library, https://git.ligo.org/lscsoft/lalsuite.

[296] J. D. Hunter, Matplotlib: A 2D Graphics Environment, Comput. Sci. Eng. 9, 90 (2007).

[297] M. Waskom and the Seaborn development team, mwaskom/seaborn, https://zenodo.org/record/4645478.

[298] D. Macleod et al., gwpy/gwpy: 2.0.1, https://zenodo.org/ record/3973364. 
[299] C. R. Harris et al., Array Programming with NumPy, Nature (London) 585, 357 (2020).

[300] P. Virtanen et al. (SciPy 1.0 Contributors), SciPy 1.0: Fundamental Algorithms for Scientific Computing in Python, Nat. Methods 17, 261 (2020).

[301] B. P. Abbott et al. (LIGO Scientific Collaboration, Virgo Collaboration), Effects of Waveform Model Systematics on the Interpretation of GW150914, Classical Quantum Gravity 34, 104002 (2017).

[302] T. D. Abbott et al. (LIGO Scientific Collaboration, Virgo Collaboration), Improved Analysis of GW150914 Using a Fully Spin-Precessing Waveform Model, Phys. Rev. X 6 , 041014 (2016).

[303] S. Kullback and R. A. Leibler, On Information and Sufficiency, Ann. Math. Stat. 22, 79 (1951).

[304] Our data release includes results from models with and without HMs.

[305] F. H. Shaik, J. Lange, S. E. Field, R. O’Shaughnessy, V. Varma, L. E. Kidder, H. P. Pfeiffer, and D. Wysocki, Impact of Subdominant Modes on the Interpretation of Gravitational-Wave Signals from Heavy Binary Black Hole Systems, Phys. Rev. D 101, 124054 (2020).

[306] M. Pürrer and C.-J. Haster, Gravitational Waveform Accuracy Requirements for Future Ground-Based Detectors, Phys. Rev. Research 2, 023151 (2020).

[307] V. Varma and P. Ajith, Effects of Nonquadrupole Modes in the Detection and Parameter Estimation of Black Hole Binaries with Nonprecessing Spins, Phys. Rev. D 96, 124024 (2017).

[308] J. C. Bustillo, S. Husa, A. M. Sintes, and M. Pürrer, Impact of Gravitational Radiation Higher Order Modes on Single Aligned-Spin Gravitational Wave Searches for Binary Black Holes, Phys. Rev. D 93, 084019 (2016).

[309] T. B. Littenberg, J. G. Baker, A. Buonanno, and B. J. Kelly, Systematic Biases in Parameter Estimation of Binary Black-Hole Mergers, Phys. Rev. D 87, 104003 (2013).

[310] J. C. Bustillo, P. Laguna, and D. Shoemaker, Detectability of Gravitational Waves from Binary Black Holes: Impact of Precession and Higher Modes, Phys. Rev. D 95, 104038 (2017).

[311] D. A. Brown, P. Kumar, and A. H. Nitz, Template Banks to Search for Low-Mass Binary Black Holes in Advanced Gravitational-Wave Detectors, Phys. Rev. D 87, 082004 (2013).

[312] V. Varma, P. Ajith, S. Husa, J. C. Bustillo, M. Hannam, and M. Pürrer, Gravitational-Wave Observations of Binary Black Holes: Effect of Nonquadrupole Modes, Phys. Rev. D 90, 124004 (2014).

[313] P. B. Graff, A. Buonanno, and B. S. Sathyaprakash, Missing Link: Bayesian Detection and Measurement of
Intermediate-Mass Black-Hole Binaries, Phys. Rev. D 92 , 022002 (2015).

[314] R. O'Shaughnessy, B. Farr, E. Ochsner, H.-S. Cho, V. Raymond, C. Kim, and C.-H. Lee, Parameter Estimation of Gravitational Waves from Precessing Black Hole-Neutron Star Inspirals with Higher Harmonics, Phys. Rev. D 89, 102005 (2014).

[315] S. A. Usman, J. C. Mills, and S. Fairhurst, Constraining the Inclinations of Binary Mergers from GravitationalWave Observations, Astrophys. J. 877, 82 (2019).

[316] P. Kumar, J. Blackman, S. E. Field, M. Scheel, C. R. Galley, M. Boyle, L. E. Kidder, H. P. Pfeiffer, B. Szilagyi, and S.A. Teukolsky, Constraining the Parameters of GW150914 and GW170104 with Numerical Relativity Surrogates, Phys. Rev. D 99, 124005 (2019).

[317] C. Mills and S. Fairhurst, Measuring Gravitational-Wave Higher-Order Multipoles, Phys. Rev. D 103, 024042 (2021).

[318] K. Chatziioannou, A. Klein, N. Yunes, and N. Cornish, Constructing Gravitational Waves from Generic SpinPrecessing Compact Binary Inspirals, Phys. Rev. D 95, 104004 (2017).

[319] B. Bécsy, P. Raffai, N. J. Cornish, R. Essick, J. Kanner, E. Katsavounidis, T. B. Littenberg, M. Millhouse, and S. Vitale, Parameter Estimation for Gravitational-Wave Bursts with the BayesWave Pipeline, Astrophys. J. 839, 15 (2017).

[320] F. Pannarale, R. Macas, and P. J. Sutton, Bayesian Inference Analysis of Unmodelled GravitationalWave Transients, Classical Quantum Gravity 36, 035011 (2019).

[321] F. Mosteller and R. A. Fisher, Questions and Answers, The American Statistician 2, 30 (1948).

Correction: Table VI contained two entries with conversion errors (fourth column, row 15 and twelfth column, row 38) and has been fixed.

Second Correction: Table VI contained 11 further entries with conversion errors (row 7, col. 11; row 8, col. 5 and col. 11; row 9 , col. 7 ; row 18 , col. 2 ; row 20 , col. 2 ; row 29 , col. 7 ; row 34 , col. 8; row 35, col. 9; row 36, col. 7; row 39, col. 7) and has been fixed.

R. Abbott, ${ }^{1}$ T. D. Abbott,${ }^{2}$ S. Abraham,${ }^{3}$ F. Acernese, ${ }^{4,5}$ K. Ackley,${ }^{6}$ A. Adams, ${ }^{7}$ C. Adams, ${ }^{8}$ R. X. Adhikari, ${ }^{1}$ V. B. Adya ${ }^{9}$ C. Affeldt, ${ }^{10,11}$ M. Agathos, ${ }^{12,13}$ K. Agatsuma, ${ }^{14}$ N. Aggarwal, ${ }^{15}$ O. D. Aguiar, ${ }^{16}$ L. Aiello, ${ }^{17,18}$ A. Ain, ${ }^{19,20}$ P. Ajith, ${ }^{21}$ S. Akcay, ${ }^{13,22}$ G. Allen, ${ }^{23}$ A. Allocca, ${ }^{19}$ P. A. Altin, ${ }^{9}$ A. Amato, ${ }^{24}$ S. Anand, ${ }^{1}$ A. Ananyeva, ${ }^{1}$ S. B. Anderson, ${ }^{1}$ W. G. Anderson, ${ }^{25}$ S. V. Angelova, ${ }^{26}$ S. Ansoldi, ${ }^{27,28}$ J. M. Antelis, ${ }^{29}$ S. Antier, ${ }^{30}$ S. Appert, ${ }^{1}$ K. Arai, ${ }^{1}$ M. C. Araya, ${ }^{1}$ J. S. Areeda, ${ }^{31}$ M. Arène, ${ }^{30}$ N. Arnaud, ${ }^{32,33}$ S. M. Aronson, ${ }^{34}$ K. G. Arun, ${ }^{35}$ Y. Asali, ${ }^{36}$ S. Ascenzi, ${ }^{17,37}$ G. Ashton, ${ }^{6}$ 
S. M. Aston, ${ }^{8}$ P. Astone,${ }^{38}$ F. Aubin, ${ }^{39}$ P. Aufmuth, ${ }^{10,11}$ K. AultONeal, ${ }^{29}$ C. Austin, ${ }^{2}$ V. Avendano, ${ }^{40}$ S. Babak,${ }^{30}$ F. Badaracco, ${ }^{17,18}$ M. K. M. Bader, ${ }^{41}$ S. Bae, ${ }^{42}$ A. M. Baer, ${ }^{7}$ S. Bagnasco, ${ }^{43}$ J. Baird, ${ }^{30}$ M. Ball ${ }^{44}$ G. Ballardin, ${ }^{33}$ S. W. Ballmer, ${ }^{45}$ A. Bals, ${ }^{29}$ A. Balsamo, ${ }^{7}$ G. Baltus, ${ }^{46}$ S. Banagiri, ${ }^{47}$ D. Bankar, ${ }^{3}$ R. S. Bankar, ${ }^{3}$ J. C. Barayoga, ${ }^{1}$ C. Barbieri, ${ }^{48,49,50}$ B. C. Barish, ${ }^{1}$ D. Barker, ${ }^{51}$ P. Barneo, ${ }^{52}$ S. Barnum,${ }^{53}$ F. Barone,${ }^{54,5}$ B. Barr, ${ }^{55}$ L. Barsotti, ${ }^{53}$ M. Barsuglia, ${ }^{30}$ D. Barta, ${ }^{56}$ J. Bartlett, ${ }^{51}$ I. Bartos,${ }^{34}$ R. Bassiri, ${ }^{57}$ A. Basti, ${ }^{20,19}$ M. Bawaj, ${ }^{58,59}$ J. C. Bayley, ${ }^{55}$ M. Bazzan,${ }^{60,61}$ B. R. Becher ${ }^{62}$ B. Bécsy ${ }^{63}$ V. M. Bedakihale,${ }^{64}$ M. Bejger, ${ }^{65}$ I. Belahcene, ${ }^{32}$ D. Beniwal, ${ }^{66}$ M. G. Benjamin, ${ }^{29}$ T. F. Bennett, ${ }^{67}$ J. D. Bentley, ${ }^{14}$ F. Bergamin, ${ }^{10,11}$ B. K. Berger, ${ }^{57}$ G. Bergmann, ${ }^{10,11}$ S. Bernuzzi, ${ }^{13}$ C. P. L. Berry, ${ }^{15}$ D. Bersanetti, ${ }^{68}$ A. Bertolini, ${ }^{41}$ J. Betzwieser, ${ }^{8}$ R. Bhandare, ${ }^{69}$ A. V. Bhandari, ${ }^{3}$ D. Bhattacharjee, ${ }^{70}$ J. Bidler, ${ }^{31}$ I. A. Bilenko, ${ }^{71}$ G. Billingsley, ${ }^{1}$ R. Birney, ${ }^{72}$ O. Birnholtz, ${ }^{73}$ S. Biscans, ${ }^{1,53}$ M. Bischi, ${ }^{74,75}$ S. Biscoveanu, ${ }^{53}$ A. Bisht,${ }^{10,11}$ M. Bitossi, ${ }^{33,19}$ M.-A. Bizouard, ${ }^{76}$ J. K. Blackburn, ${ }^{1}$ J. Blackman, ${ }^{77}$ C. D. Blair, ${ }^{78}$ D. G. Blair, ${ }^{78}$ R. M. Blair,${ }^{51}$ O. Blanch, ${ }^{79}$ F. Bobba, ${ }^{80,81}$ N. Bode ${ }^{10,11}$ M. Boer ${ }^{76}$ Y. Boetzel,${ }^{82}$ G. Bogaert, ${ }^{76}$ M. Boldrini ${ }^{83,38}$ F. Bondu, ${ }^{84}$ E. Bonilla, ${ }^{57}$ R. Bonnand,${ }^{39}$ P. Booker, ${ }^{10,11}$ B. A. Boom,${ }^{41}$ R. Bork,${ }^{1}$ V. Boschi, ${ }^{19}$ S. Bose,${ }^{3}$ V. Bossilkov ${ }^{78}$ V. Boudart, ${ }^{46}$ Y. Bouffanais ${ }^{60,61}$ A. Bozzi ${ }^{33}$ C. Bradaschia, ${ }^{19}$ P. R. Brady, ${ }^{25}$ A. Bramley, ${ }^{8}$ M. Branchesi, ${ }^{17,18}$ J. E. Brau,${ }^{44}$ M. Breschi, ${ }^{13}$ T. Briant,${ }^{85}$ J. H. Briggs,${ }^{55}$ F. Brighenti, ${ }^{7475}$ A. Brillet, ${ }^{76}$ M. Brinkmann, ${ }^{10,11}$ P. Brockill, ${ }^{25}$ A. F. Brooks, ${ }^{1}$ J. Brooks, ${ }^{33}$ D. D. Brown, ${ }^{66}$ S. Brunett, ${ }^{1}$ G. Bruno, ${ }^{86}$ R. Bruntz, ${ }^{7}$ A. Buikema, ${ }^{53}$ T. Bulik, ${ }^{87}$ H. J. Bulten, ${ }^{41,88}$ A. Buonanno, ${ }^{89,90}$ R. Buscicchio, ${ }^{14}$ D. Buskulic,${ }^{39}$

R. L. Byer, ${ }^{57}$ M. Cabero, ${ }^{10,11}$ L. Cadonati, ${ }^{91}$ M. Caesar, ${ }^{92}$ G. Cagnoli, ${ }^{24}$ C. Cahillane, ${ }^{1}$ J. Calderón Bustillo, ${ }^{6}$

J. D. Callaghan, ${ }^{55}$ T. A. Callister, ${ }^{93}$ E. Calloni, ${ }^{94,5}$ J. B. Camp,${ }^{95}$ M. Canepa,${ }^{96,68}$ K. C. Cannon, ${ }^{97}$ H. Cao, ${ }^{66}$ J. Cao, ${ }^{98}$ G. Carapella, ${ }^{80,81}$ F. Carbognani, ${ }^{33}$ M. F. Carney, ${ }^{15}$ M. Carpinelli, ${ }^{99,100}$ G. Carullo, ${ }^{20,19}$ T. L. Carver, ${ }^{101}$ J. Casanueva Diaz, ${ }^{33}$ C. Casentini, ${ }^{102,37}$ S. Caudill, ${ }^{41}$ M. Cavaglià, ${ }^{70}$ F. Cavalier,${ }^{32}$ R. Cavalieri, ${ }^{33}$ G. Cella, ${ }^{19}$ P. Cerdá-Durán, ${ }^{103}$ E. Cesarini, ${ }^{37}$

W. Chaibi, ${ }^{76}$ K. Chakravarti, ${ }^{3}$ C.-L. Chan, ${ }^{104}$ C. Chan, ${ }^{97}$ K. Chandra, ${ }^{105}$ P. Chanial, ${ }^{33}$ S. Chao, ${ }^{106}$ P. Charlton, ${ }^{107}$

E. A. Chase,${ }^{15}$ E. Chassande-Mottin, ${ }^{30}$ D. Chatterjee, ${ }^{25}$ D. Chattopadhyay, ${ }^{108}$ M. Chaturvedi, ${ }^{69}$ K. Chatziioannou, ${ }^{93}$

A. Chen, ${ }^{104}$ H. Y. Chen, ${ }^{109}$ X. Chen, ${ }^{78}$ Y. Chen, ${ }^{77}$ H.-P. Cheng, ${ }^{34}$ C. K. Cheong, ${ }^{104}$ H. Y. Chia, ${ }^{34}$ F. Chiadini, ${ }^{10,81}$ R. Chierici, ${ }^{111}$ A. Chincarini,${ }^{68}$ A. Chiummo, ${ }^{33}$ G. Cho, ${ }^{112}$ H. S. Cho, ${ }^{113}$ M. Cho,${ }^{90}$ S. Choate, ${ }^{92}$ N. Christensen, ${ }^{76}$ Q. Chu ${ }^{78}$ S. Chua, ${ }^{85}$ K. W. Chung, ${ }^{114}$ S. Chung, ${ }^{78}$ G. Ciani, ${ }^{60,61}$ P. Ciecielag, ${ }^{65}$ M. Cieślar, ${ }^{65}$ M. Cifaldi, ${ }^{102,37}$ A. A. Ciobanu ${ }^{66}$ R. Ciolfi, ${ }^{115,61}$ F. Cipriano, ${ }^{76}$ A. Cirone, ${ }^{96,68}$ F. Clara, ${ }^{51}$ E. N. Clark,${ }^{116}$ J. A. Clark, ${ }^{91}$ L. Clarke, ${ }^{117}$ P. Clearwater,${ }^{118}$ S. Clesse, ${ }^{86}$ F. Cleva, ${ }^{76}$ E. Coccia, ${ }^{17,18}$ P.-F. Cohadon, ${ }^{85}$ D. E. Cohen, ${ }^{32}$ M. Colleoni, ${ }^{119}$ C. G. Collette, ${ }^{120}$ C. Collins, ${ }^{14}$ M. Colpi,${ }^{48,49}$ M. Constancio Jr., ${ }^{16}$ L. Conti, ${ }^{61}$ S. J. Cooper, ${ }^{14}$ P. Corban, ${ }^{8}$ T. R. Corbitt, ${ }^{2}$ I. Cordero-Carrión, ${ }^{121}$ S. Corezzi, ${ }^{59,58}$ K. R. Corley, ${ }^{36}$ N. Cornish, ${ }^{63}$ D. Corre, ${ }^{32}$ A. Corsi,${ }^{122}$ S. Cortese, ${ }^{33}$ C. A. Costa, ${ }^{16}$ R. Cotesta, ${ }^{89}$ M. W. Coughlin, ${ }^{47,1}$ S. B. Coughlin, ${ }^{15,101}$ J.-P. Coulon, ${ }^{76}$ S. T. Countryman, ${ }^{36}$ B. Cousins, ${ }^{123}$ P. Couvares, ${ }^{1}$ P. B. Covas, ${ }^{119}$ D. M. Coward, ${ }^{78}$ M. J. Cowart, ${ }^{8}$ D. C. Coyne, ${ }^{1}$ R. Coyne, ${ }^{124}$ J. D. E. Creighton, ${ }^{25}$ T. D. Creighton, ${ }^{125}$ M. Croquette ${ }^{85}$

S. G. Crowder, ${ }^{126}$ J. R. Cudell, ${ }^{46}$ T. J. Cullen, ${ }^{2}$ A. Cumming, ${ }^{55}$ R. Cummings, ${ }^{55}$ L. Cunningham, ${ }^{55}$ E. Cuoco, ${ }^{33,127}$ M. Curyło, ${ }^{87}$ T. Dal Canton, ${ }^{32,89}$ G. Dálya ${ }^{128}$ A. Dana, ${ }^{57}$ L. M. DaneshgaranBajastani, ${ }^{67}$ B. D’Angelo, ${ }^{96,68}$ B. Danila, ${ }^{129}$ S. L. Danilishin, ${ }^{130}$ S. D'Antonio, ${ }^{37}$ K. Danzmann, ${ }^{10,11}$ C. Darsow-Fromm, ${ }^{131}$ A. Dasgupta, ${ }^{64}$ L. E. H. Datrier, ${ }^{55}$ V. Dattilo, ${ }^{33}$ I. Dave,${ }^{69}$ M. Davier, ${ }^{32}$ G. S. Davies ${ }^{132}$ D. Davis, ${ }^{1}$ E. J. Daw, ${ }^{133}$ R. Dean, ${ }^{92}$ D. DeBra, ${ }^{57}$ M. Deenadayalan, ${ }^{3}$ J. Degallaix, ${ }^{134}$ M. De Laurentis,${ }^{94,5}$ S. Deléglise, ${ }^{85}$ V. Del Favero, ${ }^{135}$ F. De Lillo, ${ }^{86}$ N. De Lillo, ${ }^{55}$ W. Del Pozzo, ${ }^{20,19}$ L. M. DeMarchi, ${ }^{15}$ F. De Matteis, ${ }^{102,37}$ V. D'Emilio, ${ }^{101}$ N. Demos, ${ }^{53}$ T. Denker, ${ }^{10,11}$ T. Dent, ${ }^{132}$ A. Depasse, ${ }^{86}$ R. De Pietri, ${ }^{136,137}$ R. De Rosa, ${ }^{94,5}$ C. De Rossi, ${ }^{33}$ R. DeSalvo, ${ }^{138,81}$ O. de Varona, ${ }^{10,11}$ S. Dhurandhar, ${ }^{3}$ M. C. Díaz, ${ }^{125}$ M. Diaz-Ortiz Jr., ${ }^{34}$ N. A. Didio, ${ }^{45}$ T. Dietrich,${ }^{41}$ L. Di Fiore,${ }^{5}$ C. DiFronzo,${ }^{14}$ C. Di Giorgio,${ }^{80,81}$ F. Di Giovanni, ${ }^{103}$ M. Di Giovanni, ${ }^{139,140}$ T. Di Girolamo,,${ }^{94,5}$ A. Di Lieto, ${ }^{20,19}$ B. Ding, ${ }^{120}$ S. Di Pace, ${ }^{83,38}$ I. Di Palma ${ }^{83,38}$ F. Di Renzo, ${ }^{20,19}$ A. K. Divakarla, ${ }^{34}$ A. Dmitriev, ${ }^{14}$ Z. Doctor, ${ }^{44}$ L. D'Onofrio, ${ }^{94,5}$ F. Donovan, ${ }^{53}$ K. L. Dooley, ${ }^{101}$ S. Doravari, ${ }^{3}$ I. Dorrington, ${ }^{101}$ T. P. Downes,${ }^{25}$ M. Drago, ${ }^{17,18}$ J. C. Driggers, ${ }^{51}$ Z. Du, ${ }^{98}$ J.-G. Ducoin, ${ }^{32}$ P. Dupej, ${ }^{55}$ O. Durante,,${ }^{80,81}$ D. D’Urso, ${ }^{99,100}$ P.-A. Duverne, ${ }^{32}$ S. E. Dwyer, ${ }^{51}$ P. J. Easter, ${ }^{6}$ G. Eddolls, ${ }^{55}$ B. Edelman, ${ }^{44}$ T. B. Edo, ${ }^{133}$ O. Edy, ${ }^{141}$ A. Effler, ${ }^{8}$ J. Eichholz, ${ }^{9}$ S. S. Eikenberry ${ }^{34}$ M. Eisenmann, ${ }^{39}$ R. A. Eisenstein, ${ }^{53}$ A. Ejlli, ${ }^{101}$ L. Errico, ${ }^{94,5}$ R. C. Essick, ${ }^{109}$ H. Estellés, ${ }^{119}$ D. Estevez, ${ }^{39}$ Z. B. Etienne, ${ }^{142}$ T. Etzel, ${ }^{1}$ M. Evans, ${ }^{53}$ T. M. Evans, ${ }^{8}$ B. E. Ewing, ${ }^{123}$ V. Fafone, ${ }^{102,37,17}$ H. Fair, ${ }^{45}$ S. Fairhurst, ${ }^{101}$ X. Fan, ${ }^{98}$ A. M. Farah, ${ }^{109}$ S. Farinon, ${ }^{68}$ B. Farr, ${ }^{44}$ W. M. Farr, ${ }^{143,93}$ E. J. Fauchon-Jones, ${ }^{101}$ M. Favata, ${ }^{40}$ M. Fays, ${ }^{46,133}$ M. Fazio, ${ }^{144}$ J. Feicht, ${ }^{1}$ M. M. Fejer, ${ }^{57}$ F. Feng,${ }^{30}$ E. Fenyvesi, ${ }^{56,145}$ D. L. Ferguson, ${ }^{91}$ A. Fernandez-Galiana, ${ }^{53}$ I. Ferrante, ${ }^{20,19}$ T. A. Ferreira ${ }^{16}$ F. Fidecaro, ${ }^{20,19}$ P. Figura, ${ }^{87}$ I. Fiori, ${ }^{33}$ D. Fiorucci ${ }^{17,18}$ M. Fishbach,${ }^{109}$ R. P. Fisher, ${ }^{7}$ J. M. Fishner, ${ }^{53}$ R. Fittipaldi, ${ }^{146,81}$ M. Fitz-Axen, ${ }^{47}$ V. Fiumara, ${ }^{147,81}$ R. Flaminio, ${ }^{39,148}$ E. Floden, ${ }^{47}$ E. Flynn, ${ }^{31}$ H. Fong, ${ }^{97}$ J. A. Font, ${ }^{103,149}$ P. W. F. Forsyth, ${ }^{9}$ J.-D. Fournier, ${ }^{76}$ S. Frasca, ${ }^{83,38}$ F. Frasconi, ${ }^{19}$ Z. Frei, ${ }^{128}$ A. Freise, ${ }^{14}$ R. Frey, ${ }^{44}$ V. Frey, ${ }^{32}$ P. Fritschel, ${ }^{53}$ V. V. Frolov, ${ }^{8}$ 
G. G. Fronzé, ${ }^{43}$ P. Fulda, ${ }^{34}$ M. Fyffe, ${ }^{8}$ H. A. Gabbard,${ }^{55}$ B. U. Gadre, ${ }^{89}$ S. M. Gaebel, ${ }^{14}$ J. R. Gair, ${ }^{89}$ J. Gais,${ }^{104}$ S. Galaudage, ${ }^{6}$ R. Gamba, ${ }^{13}$ D. Ganapathy, ${ }^{53}$ A. Ganguly, ${ }^{21}$ S. G. Gaonkar, ${ }^{3}$ B. Garaventa, ${ }^{68,96}$ C. García-Quirós, ${ }^{119}$ F. Garufi, ${ }^{94,5}$ B. Gateley, ${ }^{51}$ S. Gaudio, ${ }^{29}$ V. Gayathri, ${ }^{34}$ G. Gemme,${ }^{68}$ A. Gennai, ${ }^{19}$ D. George, ${ }^{23}$ J. George, ${ }^{69}$ R. N. George, ${ }^{150}$ L. Gergely, ${ }^{129}$ S. Ghonge, ${ }^{91}$ Abhirup Ghosh, ${ }^{89}$ Archisman Ghosh, ${ }^{41,151-} 153$ S. Ghosh,${ }^{25,40}$ B. Giacomazzo, ${ }^{48,49,50}$ L. Giacoppo, ${ }^{83,38}$ J. A. Giaime, ${ }^{2,8}$ K. D. Giardina, ${ }^{8}$ D. R. Gibson, ${ }^{72}$ C. Gier, ${ }^{26}$ K. Gill, ${ }^{36}$ P. Giri, ${ }^{1920}$ J. Glanzer, ${ }^{2}$ A. E. Gleckl, ${ }^{31}$ P. Godwin, ${ }^{123}$ E. Goetz, ${ }^{154}$ R. Goetz, ${ }^{34}$ N. Gohlke, ${ }^{10,11}$ B. Goncharov, ${ }^{6}$ G. González, ${ }^{2}$ A. Gopakumar, ${ }^{155}$ S. E. Gossan, ${ }^{1}$ M. Gosselin, ${ }^{20,19}$ R. Gouaty, ${ }^{39}$ B. Grace, ${ }^{9}$ A. Grado, ${ }^{156,5}$ M. Granata, ${ }^{134}$ V. Granata,${ }^{80}$ A. Grant,${ }^{55}$ S. Gras, ${ }^{53}$ P. Grassia, ${ }^{1}$ C. Gray, ${ }^{51}$ R. Gray,${ }^{55}$ G. Greco,${ }^{74,75}$ A. C. Green,${ }^{34}$ R. Green, ${ }^{101}$ E. M. Gretarsson, ${ }^{29}$ H. L. Griggs,${ }^{91}$ G. Grignani, ${ }^{59,58}$ A. Grimaldi, ${ }^{139,140}$ E. Grimes, ${ }^{29}$ S. J. Grimm, ${ }^{17,18}$ H. Grote, ${ }^{101}$ S. Grunewald, ${ }^{89}$ P. Gruning, ${ }^{32}$ J. G. Guerrero, ${ }^{31}$ G. M. Guidi, ${ }^{74,75}$ A. R. Guimaraes, ${ }^{2}$ G. Guixé, ${ }^{52}$ H. K. Gulati, ${ }^{64}$ Y. Guo, ${ }^{41}$ Anchal Gupta, ${ }^{1}$ Anuradha Gupta, ${ }^{123}$ P. Gupta, ${ }^{41,157}$ E. K. Gustafson, ${ }^{1}$ R. Gustafson, ${ }^{158}$ F. Guzman, ${ }^{116}$ L. Haegel, ${ }^{30}$ O. Halim, ${ }^{18,17}$ E. D. Hall, ${ }^{53}$ E. Z. Hamilton, ${ }^{101}$ G. Hammond, ${ }^{55}$ M. Haney, ${ }^{82}$ M. M. Hanke, ${ }^{10,11}$ J. Hanks,${ }^{51}$ C. Hanna, ${ }^{123}$ M. D. Hannam, ${ }^{101}$ O. A. Hannuksela, ${ }^{104}$ O. Hannuksela, ${ }^{157,41}$ H. Hansen, ${ }^{51}$ T. J. Hansen ${ }^{29}$ J. Hanson, ${ }^{8}$ T. Harder, ${ }^{76}$ T. Hardwick, ${ }^{2}$ K. Haris, ${ }^{41,157,21}$ J. Harms, ${ }^{17,18}$ G. M. Harry, ${ }^{159}$ I. W. Harry, ${ }^{141}$ D. Hartwig, ${ }^{131}$ R. K. Hasskew, ${ }^{8}$ C.-J. Haster, ${ }^{53}$ K. Haughian, ${ }^{55}$ F. J. Hayes,${ }^{55}$ J. Healy, ${ }^{135}$ A. Heidmann ${ }^{85}$ M. C. Heintze,${ }^{8}$ J. Heinze,${ }^{10,11}$ J. Heinzel, ${ }^{160}$ H. Heitmann, ${ }^{76}$ F. Hellman, ${ }^{161}$

P. Hello, ${ }^{32}$ A. F. Helmling-Cornell,${ }^{44}$ G. Hemming,${ }^{33}$ M. Hendry, ${ }^{55}$ I. S. Heng ${ }^{55}$ E. Hennes,${ }^{41}$ J. Hennig, ${ }^{10,11}$

M. H. Hennig, ${ }^{10,11}$ F. Hernandez Vivanco, ${ }^{6}$ M. Heurs, ${ }^{10,11}$ S. Hild,${ }^{130}$ P. Hill,${ }^{26}$ A. S. Hines,${ }^{116}$ S. Hochheim, ${ }^{10,11}$ E. Hofgard, ${ }^{57}$ D. Hofman, ${ }^{134}$ J. N. Hohmann, ${ }^{131}$ A. M. Holgado ${ }^{23}$ N. A. Holland,,${ }^{9}$ I. J. Hollows, ${ }^{133}$ Z. J. Holmes, ${ }^{66}$ K. Holt,${ }^{8}$ D. E. Holz,${ }^{109}$ P. Hopkins, ${ }^{101}$ C. Horst,${ }^{25}$ J. Hough, ${ }^{55}$ E. J. Howell, ${ }^{78}$ C. G. Hoy,${ }^{101}$ D. Hoyland, ${ }^{14}$ Y. Huang,${ }^{53}$ M. T. Hübner, ${ }^{6}$ A. D. Huddart ${ }^{117}$ E. A. Huerta, ${ }^{23}$ B. Hughey, ${ }^{29}$ V. Hui, ${ }^{39}$ S. Husa, ${ }^{119}$ S. H. Huttner, ${ }^{55}$ B. M. Hutzler, ${ }^{2}$ R. Huxford,${ }^{123}$ T. Huynh-Dinh, ${ }^{8}$ B. Idzkowski, ${ }^{87}$ A. Iess, ${ }^{102,37}$ S. Imperato, ${ }^{15}$ H. Inchauspe,${ }^{34}$ C. Ingram, ${ }^{66}$ G. Intini,${ }^{83,38}$ M. Isi, ${ }^{53}$ B. R. Iyer, ${ }^{21}$ V. JaberianHamedan, ${ }^{78}$ T. Jacqmin,${ }^{85}$ S. J. Jadhav, ${ }^{162}$ S. P. Jadhav, ${ }^{3}$ A. L. James, ${ }^{101}$ K. Jani, ${ }^{91}$ K. Janssens, ${ }^{163}$ N. N. Janthalur, ${ }^{162}$ P. Jaranowski, ${ }^{164}$ D. Jariwala, ${ }^{34}$ R. Jaume, ${ }^{119}$ A. C. Jenkins, ${ }^{114}$ M. Jeunon, ${ }^{47}$ J. Jiang, ${ }^{34}$ G. R. Johns, ${ }^{7}$ N. K. Johnson-McDaniel, ${ }^{12}$ A. W. Jones, ${ }^{14}$ D. I. Jones, ${ }^{165}$ J. D. Jones,${ }^{51}$ P. Jones, ${ }^{14}$ R. Jones, ${ }^{55}$ R. J. G. Jonker, ${ }^{41}$ L. Ju, ${ }^{78}$ J. Junker, ${ }^{10,11}$ C. V. Kalaghatgi, ${ }^{101}$ V. Kalogera, ${ }^{15}$ B. Kamai, ${ }^{1}$ S. Kandhasamy, ${ }^{3}$ G. Kang, ${ }^{42}$ J. B. Kanner, ${ }^{1}$ S. J. Kapadia, ${ }^{21}$ D. P. Kapasi, ${ }^{9}$ C. Karathanasis, ${ }^{79}$ S. Karki, ${ }^{70}$ R. Kashyap, ${ }^{123}$ M. Kasprzack, ${ }^{1}$ W. Kastaun, ${ }^{10,11}$ S. Katsanevas, ${ }^{33}$

E. Katsavounidis, ${ }^{53}$ W. Katzman, ${ }^{8}$ K. Kawabe,${ }^{51}$ F. Kéfélian, ${ }^{76}$ D. Keitel, ${ }^{119}$ J. S. Key, ${ }^{166}$ S. Khadka, ${ }^{57}$ F. Y. Khalili, ${ }^{71}$ I. Khan, ${ }^{17,37}$ S. Khan, ${ }^{101}$ E. A. Khazanov, ${ }^{167}$ N. Khetan, ${ }^{17,18}$ M. Khursheed ${ }^{69}$ N. Kijbunchoo, ${ }^{9}$ C. Kim, ${ }^{168}$ G. J. Kim, ${ }^{91}$ J. C. Kim, ${ }^{169}$ K. Kim, ${ }^{170}$ W. S. Kim, ${ }^{171}$ Y.-M. Kim, ${ }^{172}$ C. Kimball, ${ }^{15}$ P. J. King, ${ }^{51}$ M. Kinley-Hanlon, ${ }^{55}$ R. Kirchhoff, ${ }^{10,11}$ J. S. Kissel,${ }^{51}$ L. Kleybolte, ${ }^{131}$ S. Klimenko, ${ }^{34}$ T. D. Knowles, ${ }^{142}$ E. Knyazev, ${ }^{53}$ P. Koch, ${ }^{10,11}$ S. M. Koehlenbeck,,${ }^{10,11}$ G. Koekoek, ${ }^{41,173}$ S. Koley, ${ }^{41}$ M. Kolstein, ${ }^{79}$ K. Komori, ${ }^{53}$ V. Kondrashov, ${ }^{1}$ A. Kontos, ${ }^{62}$ N. Koper, ${ }^{10,11}$ M. Korobko, ${ }^{131}$ W. Z. Korth, ${ }^{1}$ M. Kovalam, ${ }^{78}$ D. B. Kozak, ${ }^{1}$ C. Krämer, ${ }^{10,11}$ V. Kringel,,${ }^{10,11}$ N. V. Krishnendu, ${ }^{10,11}$ A. Królak, ${ }^{174,175}$ G. Kuehn, ${ }^{10,11}$ A. Kumar, ${ }^{162}$ P. Kumar, ${ }^{176}$ Rahul Kumar, ${ }^{51}$ Rakesh Kumar, ${ }^{64}$ K. Kuns, ${ }^{53}$ S. Kwang, ${ }^{25}$ B. D. Lackey ${ }^{89}$ D. Laghi, ${ }^{20,19}$ E. Lalande, ${ }^{177}$ T. L. Lam, ${ }^{104}$ A. Lamberts, ${ }^{76,178}$ M. Landry,${ }^{51}$ B. B. Lane, ${ }^{53}$ R. N. Lang, ${ }^{53}$ J. Lange, ${ }^{135}$ B. Lantz ${ }^{57}$ R. K. Lanza, ${ }^{53}$ I. La Rosa,${ }^{39}$ A. Lartaux-Vollard ${ }^{32}$ P. D. Lasky, ${ }^{6}$ M. Laxen, ${ }^{8}$ A. Lazzarini, ${ }^{1}$ C. Lazzaro, ${ }^{61,60}$ P. Leaci, ${ }^{83,38}$ S. Leavey, ${ }^{10,11}$ Y. K. Lecoeuche, ${ }^{51}$ H. M. Lee, ${ }^{170}$ H. W. Lee, ${ }^{169}$ J. Lee, ${ }^{112}$ K. Lee, ${ }^{57}$ J. Lehmann,,${ }^{10,11}$ E. Leon, ${ }^{31}$ N. Leroy, ${ }^{32}$ N. Letendre, ${ }^{39}$ Y. Levin, ${ }^{6}$ A. Li,${ }^{1}$ J. Li ${ }^{98}$ K. J. L. Li,${ }^{104}$ T. G. F. Li,${ }^{104}$ X. Li,${ }^{77}$ F. Linde, ${ }^{179,41}$ S. D. Linker, ${ }^{67}$ J. N. Linley, ${ }^{55}$ T. B. Littenberg, ${ }^{180}$ J. Liu, ${ }^{10,11}$ X. Liu, ${ }^{25}$ M. Llorens-Monteagudo, ${ }^{103}$ R. K. L. Lo, ${ }^{1}$ A. Lockwood, ${ }^{181}$

L. T. London, ${ }^{53}$ A. Longo, ${ }^{182,183}$ M. Lorenzini, ${ }^{102,37}$ V. Loriette, ${ }^{184}$ M. Lormand, ${ }^{8}$ G. Losurdo, ${ }^{19}$ J. D. Lough, ${ }^{10,11}$ C. O. Lousto, ${ }^{135}$ G. Lovelace, ${ }^{31}$ H. Lück, ${ }^{10,11}$ D. Lumaca, ${ }^{102,37}$ A. P. Lundgren, ${ }^{141}$ Y. Ma, ${ }^{77}$ R. Macas, ${ }^{101}$ M. MacInnis,${ }^{53}$ D. M. Macleod, ${ }^{101}$ I. A. O. MacMillan, ${ }^{1}$ A. Macquet, ${ }^{76}$ I. Magaña Hernandez, ${ }^{25}$ F. Magaña-Sandoval,${ }^{34}$ C. Magazzù,${ }^{19}$ R. M. Magee, ${ }^{123}$ E. Majorana, ${ }^{38}$ I. Maksimovic, ${ }^{184}$ S. Maliakal, ${ }^{1}$ A. Malik, ${ }^{69}$ N. Man,${ }^{76}$ V. Mandic, ${ }^{47}$ V. Mangano,${ }^{83,38}$ G. L. Mansell, ${ }^{51,53}$ M. Manske, ${ }^{25}$ M. Mantovani, ${ }^{33}$ M. Mapelli, ${ }^{60,61}$ F. Marchesoni, ${ }^{185,58}$ F. Marion, ${ }^{39}$ S. Márka, ${ }^{36}$ Z. Márka, ${ }^{36}$ C. Markakis, ${ }^{12}$ A. S. Markosyan, ${ }^{57}$ A. Markowitz, ${ }^{1}$ E. Maros, ${ }^{1}$ A. Marquina, ${ }^{121}$ S. Marsat ${ }^{30}$ F. Martelli, ${ }^{74,75}$ I. W. Martin, ${ }^{55}$ R. M. Martin, ${ }^{40}$ M. Martinez, ${ }^{79}$ V. Martinez, ${ }^{24}$ D. V. Martynov,${ }^{14}$ H. Masalehdan, ${ }^{131}$ K. Mason,${ }^{53}$ E. Massera, ${ }^{133}$ A. Masserot, ${ }^{39}$ T. J. Massinger, ${ }^{53}$ M. Masso-Reid ${ }^{55}$ S. Mastrogiovanni ${ }^{30}$ A. Matas, ${ }^{89}$ M. Mateu-Lucena, ${ }^{119}$ F. Matichard, ${ }^{1,53}$ M. Matiushechkina, ${ }^{10,11}$ N. Mavalvala, ${ }^{53}$ E. Maynard, ${ }^{2}$ J. J. McCann,${ }^{78}$ R. McCarthy, ${ }^{51}$ D. E. McClelland, ${ }^{9}$ S. McCormick, ${ }^{8}$ L. McCuller, ${ }^{53}$ S. C. McGuire, ${ }^{186}$ C. McIsaac, ${ }^{141}$ J. McIver, ${ }^{154}$ D. J. McManus, ${ }^{9}$ T. McRae, ${ }^{9}$ S. T. McWilliams, ${ }^{142}$ 
D. Meacher, ${ }^{25}$ G. D. Meadors, ${ }^{6}$ M. Mehmet,${ }^{10,11}$ A. K. Mehta, ${ }^{89}$ A. Melatos, ${ }^{118}$ D. A. Melchor, ${ }^{31}$ G. Mendell, ${ }^{51}$ A. Menendez-Vazquez, ${ }^{79}$ R. A. Mercer, ${ }^{25}$ L. Mereni, ${ }^{134}$ K. Merfeld, ${ }^{44}$ E. L. Merilh, ${ }^{51}$ J. D. Merritt, ${ }^{44}$ M. Merzougui, ${ }^{76}$ S. Meshkov, ${ }^{1}$ C. Messenger ${ }^{55}$ C. Messick,${ }^{150}$ R. Metzdorff, ${ }^{85}$ P. M. Meyers,${ }^{118}$ F. Meylahn, ${ }^{10,11}$ A. Mhaske, ${ }^{3}$ A. Miani, ${ }^{139,140}$ H. Miao, ${ }^{14}$ I. Michaloliakos, ${ }^{34}$ C. Michel,${ }^{134}$ H. Middleton, ${ }^{118}$ L. Milano, ${ }^{94,5}$ A. L. Miller, ${ }^{34,86}$ M. Millhouse, ${ }^{118}$ J. C. Mills, ${ }^{101}$ E. Milotti, ${ }^{187,28}$ M. C. Milovich-Goff, ${ }^{67}$ O. Minazzoli, ${ }^{76,188}$ Y. Minenkov, ${ }^{37}$ Ll. M. Mir, ${ }^{79}$ A. Mishkin, ${ }^{34}$ C. Mishra, ${ }^{189}$ T. Mistry, ${ }^{133}$ S. Mitra, ${ }^{3}$ V. P. Mitrofanov, ${ }^{71}$ G. Mitselmakher, ${ }^{34}$ R. Mittleman,${ }^{53}$ G. Mo,${ }^{53}$ K. Mogushi, ${ }^{70}$ S. R. P. Mohapatra,${ }^{53}$ S. R. Mohite, ${ }^{25}$ I. Molina, ${ }^{31}$ M. Molina-Ruiz, ${ }^{161}$ M. Mondin, ${ }^{67}$ M. Montani,${ }^{74,75}$ C. J. Moore,${ }^{14}$ D. Moraru,${ }^{51}$ F. Morawski,${ }^{65}$ G. Moreno, ${ }^{51}$ S. Morisaki, ${ }^{97}$ B. Mours, ${ }^{190}$ C. M. Mow-Lowry, ${ }^{14}$ S. Mozzon, ${ }^{141}$ F. Muciaccia, ${ }^{83,38}$ Arunava Mukherjee,${ }^{55}$ D. Mukherjee, ${ }^{123}$ Soma Mukherjee, ${ }^{125}$ Subroto Mukherjee, ${ }^{64}$ N. Mukund,${ }^{10,11}$ A. Mullavey, ${ }^{8}$ J. Munch ${ }^{66}$ E. A. Muñiz, ${ }^{45}$ P. G. Murray, ${ }^{55}$ S. L. Nadji, ${ }^{10,11}$ A. Nagar, ${ }^{191,43,192}$ I. Nardecchia, ${ }^{102,37}$ L. Naticchioni, ${ }^{38}$ R. K. Nayak, ${ }^{193}$ B. F. Neil, ${ }^{78}$ J. Neilson, ${ }^{138,81}$ G. Nelemans, ${ }^{194}$ T. J. N. Nelson, ${ }^{8}$ M. Nery, ${ }^{10,11}$ A. Neunzert, ${ }^{166}$ A. H. Nitz, ${ }^{10,11}$ K. Y. Ng,${ }^{53}$ S. Ng, ${ }^{66}$ C. Nguyen, ${ }^{30}$ P. Nguyen, ${ }^{44}$ T. Nguyen, ${ }^{53}$ S. A. Nichols, ${ }^{2}$ S. Nissanke, ${ }^{151,41}$ F. Nocera, ${ }^{33}$ M. Noh, ${ }^{154}$ C. North, ${ }^{101}$ D. Nothard, ${ }^{195}$ L. K. Nuttall, ${ }^{141}$ J. Oberling, ${ }^{51}$ B. D. O’Brien, ${ }^{34}$ J. O’Dell, ${ }^{117}$ G. Oganesyan,,${ }^{17,18}$ G. H. Ogin, ${ }^{196}$ J. J. Oh, ${ }^{171}$ S. H. Oh, ${ }^{171}$ F. Ohme, ${ }^{10,11}$ H. Ohta, ${ }^{97}$ M. A. Okada, ${ }^{16}$ C. Olivetto, ${ }^{33}$ P. Oppermann, ${ }^{10,11}$ R. J. Oram, ${ }^{8}$ B. O'Reilly, ${ }^{8}$ R. G. Ormiston, ${ }^{47}$ L. F. Ortega,${ }^{34}$ R. O'Shaughnessy, ${ }^{135}$ S. Ossokine, ${ }^{89}$ C. Osthelder, ${ }^{1}$ D. J. Ottaway, ${ }^{66}$ H. Overmier, ${ }^{8}$ B. J. Owen, ${ }^{122}$ A. E. Pace, ${ }^{123}$ G. Pagano, ${ }^{20,19}$ M. A. Page,${ }^{78}$ G. Pagliaroli,${ }^{17,18}$ A. Pai, ${ }^{105}$ S. A. Pai, ${ }^{69}$ J. R. Palamos, ${ }^{44}$ O. Palashov, ${ }^{167}$ C. Palomba, ${ }^{38}$ H. Pan, ${ }^{106}$ P. K. Panda, ${ }^{162}$ T. H. Pang, ${ }^{41,157}$ C. Pankow, ${ }^{15}$ F. Pannarale,${ }^{83,38}$ B. C. Pant, ${ }^{69}$ F. Paoletti, ${ }_{19}$ A. Paoli, ${ }^{33}$ A. Paolone, ${ }^{38,197}$ W. Parker,${ }^{8,186}$ D. Pascucci, ${ }^{41}$ A. Pasqualetti, ${ }^{33}$ R. Passaquieti, ${ }^{20,19}$ D. Passuello, ${ }^{19}$ M. Patel, ${ }^{7}$ B. Patricelli, ${ }^{20,19}$ E. Payne, ${ }^{6}$ T. C. Pechsiri, ${ }^{34}$ M. Pedraza, ${ }^{1}$ M. Pegoraro, ${ }^{61}$ A. Pele, ${ }^{8}$ S. Penn, ${ }^{198}$ A. Perego, ${ }^{139,140}$ C. J. Perez ${ }^{51}$ C. Périgois, ${ }^{39}$ A. Perreca, ${ }^{139,140}$ S. Perriès, ${ }^{111}$ J. Petermann, ${ }^{131}$ D. Petterson, ${ }^{1}$ H. P. Pfeiffer, ${ }^{89}$ K. A. Pham, ${ }^{47}$ K. S. Phukon, ${ }^{41,179,3}$ O. J. Piccinni, ${ }^{83,38}$ M. Pichot, ${ }^{76}$ M. Piendibene,${ }^{20,19}$ F. Piergiovanni, ${ }^{74,75}$ L. Pierini, ${ }^{83,38}$ V. Pierro, ${ }^{138,81}$ G. Pillant, ${ }^{33}$ F. Pilo, ${ }^{19}$ L. Pinard, ${ }^{134}$ I. M. Pinto,${ }^{138,81,191}$ K. Piotrzkowski, ${ }^{86}$ M. Pirello, ${ }^{51}$ M. Pitkin, ${ }^{199}$ E. Placidi, ${ }^{83}$ W. Plastino, ${ }^{182,183}$ C. Pluchar, ${ }^{116}$ R. Poggiani, ${ }^{20,19}$ E. Polini, ${ }^{39}$ D. Y. T. Pong, ${ }^{104}$ S. Ponrathnam, ${ }^{3}$ P. Popolizio, ${ }^{33}$ E. K. Porter, ${ }^{30}$ A. Poverman, ${ }^{62}$ J. Powell, ${ }^{108}$ M. Pracchia, ${ }^{39}$ A. K. Prajapati, ${ }^{64}$ K. Prasai, ${ }^{57}$ R. Prasanna,${ }^{162}$ G. Pratten, ${ }^{14}$ T. Prestegard, ${ }^{25}$ M. Principe, ${ }^{138,191,81}$ G. A. Prodi, ${ }^{200,140}$ L. Prokhorov, ${ }^{14}$ P. Prosposito, ${ }^{102,37}$ L. Prudenzi, ${ }^{89}$ A. Puecher, ${ }^{41,157}$ M. Punturo, ${ }^{58}$ F. Puosi, ${ }^{19,20}$ P. Puppo, ${ }^{38}$ M. Pürrer, ${ }^{89}$ H. Qi,${ }^{101}$ V. Quetschke, ${ }^{125}$ P. J. Quinonez, ${ }^{29}$ R. Quitzow-James, ${ }^{70}$ F. J. Raab, ${ }^{51}$ G. Raaijmakers, ${ }^{151,41}$ H. Radkins, ${ }^{51}$ N. Radulesco, ${ }^{76}$ P. Raffai, ${ }^{128}$ H. Rafferty, ${ }^{201}$ S. X. Rail, ${ }^{177}$ S. Raja, ${ }^{69}$ C. Rajan, ${ }^{69}$ B. Rajbhandari, ${ }^{122}$ M. Rakhmanov, ${ }^{125}$ K. E. Ramirez, ${ }^{125}$ T. D. Ramirez,${ }^{31}$ A. Ramos-Buades,${ }^{119}$ J. Rana, ${ }^{123}$ K. Rao, ${ }^{15}$ P. Rapagnani, ${ }^{83,38}$ U. D. Rapol, ${ }^{202}$ B. Ratto, ${ }^{29}$ V. Raymond, ${ }^{101}$ M. Razzano, ${ }^{20,19}$ J. Read, ${ }^{31}$ T. Regimbau, ${ }^{39}$ L. Rei, ${ }^{68}$ S. Reid, ${ }^{26}$ D. H. Reitze, ${ }^{1,34}$ P. Rettegno, ${ }^{203,43}$ F. Ricci, ${ }^{83,38}$ C. J. Richardson, ${ }^{29}$ J. W. Richardson, ${ }^{1}$ L. Richardson, ${ }^{116}$ P. M. Ricker, ${ }^{23}$ G. Riemenschneider, ${ }^{203,43}$ K. Riles, ${ }^{158}$ M. Rizzo, ${ }^{15}$ N. A. Robertson, ${ }^{1,55}$ F. Robinet,${ }^{32}$ A. Rocchi, ${ }^{37}$ J. A. Rocha, ${ }^{31}$ S. Rodriguez, ${ }^{31}$ R. D. Rodriguez-Soto, ${ }^{29}$ L. Rolland, ${ }^{39}$ J. G. Rollins, ${ }^{1}$ V. J. Roma, ${ }^{44}$ M. Romanelli, ${ }^{84}$ R. Romano, ${ }^{4,5}$ C. L. Romel, ${ }^{51}$ A. Romero, ${ }^{79}$ I. M. Romero-Shaw, ${ }^{6}$ J. H. Romie, ${ }^{8}$ S. Ronchini, ${ }^{17,18}$ C. A. Rose, ${ }^{25}$ D. Rose, ${ }^{31}$ K. Rose, ${ }^{195}$ M. J. B. Rosell, ${ }^{150}$ D. Rosińska, ${ }^{87}$ S. G. Rosofsky, ${ }^{23}$ M. P. Ross, ${ }^{181}$ S. Rowan, ${ }^{55}$ S. J. Rowlinson, ${ }^{14}$ Santosh Roy, ${ }^{3}$ Soumen Roy, ${ }^{204}$ P. Ruggi, ${ }^{33}$ K. Ryan, ${ }^{51}$ S. Sachdev, ${ }^{123}$ T. Sadecki, ${ }^{51}$ J. Sadiq ${ }^{135}$ M. Sakellariadou, ${ }^{114}$ O. S. Salafia, ${ }^{50,49,48}$

L. Salconi, ${ }^{33}$ M. Saleem, ${ }^{35}$ A. Samajdar, ${ }^{41,157}$ E. J. Sanchez, ${ }^{1}$ J. H. Sanchez, ${ }^{31}$ L. E. Sanchez, ${ }^{1}$ N. Sanchis-Gual, ${ }^{205}$ J. R. Sanders, ${ }^{206}$ L. Sandles, ${ }^{101}$ K. A. Santiago, ${ }^{40}$ E. Santos, ${ }^{76}$ T. R. Saravanan, ${ }^{3}$ N. Sarin, ${ }^{6}$ B. Sassolas,${ }^{134}$

B. S. Sathyaprakash, ${ }^{123,101}$ O. Sauter, ${ }^{39}$ R. L. Savage, ${ }^{51}$ V. Savant, ${ }^{3}$ D. Sawant, ${ }^{105}$ S. Sayah, ${ }^{134}$ D. Schaetzl, ${ }^{1}$ P. Schale, ${ }^{44}$ M. Scheel, ${ }^{77}$ J. Scheuer, ${ }^{15}$ A. Schindler-Tyka, ${ }^{34}$ P. Schmidt, ${ }^{14}$ R. Schnabel, ${ }^{131}$ R. M. S. Schofield, ${ }^{44}$ A. Schönbeck, ${ }^{131}$ E. Schreiber, ${ }^{10,11}$ B. W. Schulte, ${ }^{10,11}$ B. F. Schutz, ${ }^{101,10}$ O. Schwarm, ${ }^{196}$ E. Schwartz, ${ }^{101}$ J. Scott, ${ }^{55}$ S. M. Scott, ${ }^{9}$ M. Seglar-Arroyo ${ }^{39}$ E. Seidel, ${ }^{23}$ D. Sellers, ${ }^{8}$ A. S. Sengupta,${ }^{204}$ N. Sennett,${ }^{89}$ D. Sentenac, ${ }^{33}$ V. Sequino, ${ }^{94,5}$ A. Sergeev, ${ }^{167}$ Y. Setyawati, ${ }^{10,11}$ T. Shaffer, ${ }^{51}$ M. S. Shahriar, ${ }^{15}$ S. Sharifi, ${ }^{2}$ A. Sharma, ${ }^{17,18}$ P. Sharma, ${ }^{69}$ P. Shawhan, ${ }^{90}$ H. Shen, ${ }^{23}$ M. Shikauchi, ${ }^{97}$ R. Shink, ${ }^{177}$ D. H. Shoemaker, ${ }^{53}$ D. M. Shoemaker, ${ }^{91}$ K. Shukla, ${ }^{161}$ S. ShyamSundar, ${ }^{69}$ M. Sieniawska, ${ }^{65}$ D. Sigg, ${ }^{51}$ L. P. Singer, ${ }^{95}$ D. Singh, ${ }^{123}$ N. Singh, ${ }^{87}$ A. Singha, ${ }^{130}$ A. Singhal, ${ }^{17,38}$ A. M. Sintes, ${ }^{119}$ V. Sipala, ${ }^{99,100}$ V. Skliris, ${ }^{101}$ B. J. J. Slagmolen, ${ }^{9}$ T. J. Slaven-Blair, ${ }^{78}$ J. Smetana, ${ }^{14}$ J. R. Smith, ${ }^{31}$ R. J. E. Smith, ${ }^{6}$ S. N. Somala, ${ }^{207}$ E. J. Son, ${ }^{171}$ K. Soni, ${ }^{3}$ S. Soni, ${ }^{2}$ B. Sorazu, ${ }^{55}$ V. Sordini, ${ }^{111}$ F. Sorrentino, ${ }^{68}$ N. Sorrentino, ${ }^{20,19}$ R. Soulard, ${ }^{76}$ T. Souradeep, ${ }^{202,3}$ E. Sowell, ${ }^{122}$ A. P. Spencer, ${ }^{55}$ M. Spera, ${ }^{60,61,15}$ A. K. Srivastava,${ }^{64}$ V. Srivastava, ${ }^{45}$ K. Staats, ${ }^{15}$ C. Stachie, ${ }^{76}$ D. A. Steer, ${ }^{30}$ J. Steinhoff, ${ }^{89}$ 
M. Steinke, ${ }^{10,11}$ J. Steinlechner, ${ }^{130,55}$ S. Steinlechner ${ }^{130}$ D. Steinmeyer, ${ }^{10,11}$ S. P. Stevenson, ${ }^{108}$ G. Stolle-McAllister, ${ }^{195}$ D. J. Stops ${ }^{14}$ M. Stover, ${ }^{195}$ K. A. Strain,${ }^{55}$ G. Stratta, ${ }^{208,75}$ A. Strunk,${ }^{51}$ R. Sturani, ${ }^{209}$ A. L. Stuver, ${ }^{92}$ J. Südbeck, ${ }^{131}$ S. Sudhagar, ${ }^{3}$ V. Sudhir, ${ }^{53}$ H. G. Suh, ${ }^{25}$ T. Z. Summerscales, ${ }^{210}$ H. Sun, ${ }^{78}$ L. Sun, ${ }^{1}$ S. Sunil, ${ }^{64}$ A. Sur, ${ }^{65}$ J. Suresh,${ }^{97}$ P. J. Sutton, ${ }^{101}$ B. L. Swinkels, ${ }^{41}$ M. J. Szczepańczyk, ${ }^{34}$ M. Tacca, ${ }^{41}$ S. C. Tait,${ }^{55}$ C. Talbot, ${ }^{6}$ A. J. Tanasijczuk, ${ }^{86}$

D. B. Tanner, ${ }^{34}$ D. Tao, ${ }^{1}$ A. Tapia,${ }^{31}$ E. N. Tapia San Martin, ${ }^{41}$ J. D. Tasson, ${ }^{160}$ R. Taylor, ${ }^{1}$ R. Tenorio, ${ }^{119}$ L. Terkowski, ${ }^{131}$ M. P. Thirugnanasambandam, ${ }^{3}$ L. M. Thomas,${ }^{14}$ M. Thomas, ${ }^{8}$ P. Thomas, ${ }^{51}$ J. E. Thompson, ${ }^{101}$ S. R. Thondapu, ${ }^{69}$

K. A. Thorne, ${ }^{8}$ E. Thrane, ${ }^{6}$ Shubhanshu Tiwari, ${ }^{82}$ Srishti Tiwari, ${ }^{155}$ V. Tiwari, ${ }^{101}$ K. Toland,${ }^{55}$ A. E. Tolley, ${ }^{141}$ M. Tonelli, ${ }^{20,19}$ Z. Tornasi, ${ }^{55}$ A. Torres-Forné ${ }^{89}$ C. I. Torrie, ${ }^{1}$ I. Tosta e Melo, ${ }^{99,100}$ D. Töyrä, ${ }^{9}$ A. T. Tran, ${ }^{126}$ A. Trapananti, ${ }^{185,58}$ F. Travasso, ${ }^{58,185}$ G. Traylor, ${ }^{8}$ M. C. Tringali, ${ }^{87}$ A. Tripathee, ${ }^{158}$ A. Trovato,${ }^{30}$ R. J. Trudeau, ${ }^{1}$ D. S. Tsai, ${ }^{106}$

K. W. Tsang, ${ }^{41,211,157}$ M. Tse, ${ }^{53}$ R. Tso, ${ }^{77}$ L. Tsukada, ${ }^{97}$ D. Tsuna, ${ }^{97}$ T. Tsutsui, ${ }^{97}$ M. Turconi, ${ }^{76}$ A. S. Ubhi, ${ }^{14}$ R. P. Udall, ${ }^{91}$ K. Ueno,${ }^{97}$ D. Ugolini, ${ }^{201}$ C. S. Unnikrishnan, ${ }^{155}$ A. L. Urban, ${ }^{2}$ S. A. Usman, ${ }^{109}$ A. C. Utina, ${ }^{130}$ H. Vahlbruch, ${ }^{10,11}$ G. Vajente, ${ }^{1}$ A. Vajpeyi, ${ }^{6}$ G. Valdes,${ }^{2}$ M. Valentini, ${ }^{139,140}$ V. Valsan, ${ }^{25}$ N. van Bakel, ${ }^{41}$ M. van Beuzekom, ${ }^{41}$ J. F. J. van den Brand, ${ }^{173,88,41}$ C. Van Den Broeck, ${ }^{157,41}$ D. C. Vander-Hyde, ${ }^{45}$ L. van der Schaaf, ${ }^{41}$ J. V. van Heijningen, ${ }^{78}$ M. Vardaro, ${ }^{179,41}$ A. F. Vargas, ${ }^{118}$ V. Varma,${ }^{77}$ S. Vass, ${ }^{1}$ M. Vasúth,${ }^{56}$ A. Vecchio,${ }^{14}$ G. Vedovato,${ }^{61}$ J. Veitch, ${ }^{55}$ P. J. Veitch ${ }^{66}$ K. Venkateswara ${ }^{181}$ J. Venneberg, ${ }^{10,11}$ G. Venugopalan, ${ }^{1}$ D. Verkindt,${ }^{39}$ Y. Verma,${ }^{69}$ D. Veske, ${ }^{36}$ F. Vetrano, ${ }^{74}$ A. Viceré, ${ }^{74,75}$ A. D. Viets, ${ }^{212}$ A. Vijaykumar, ${ }^{21}$ V. Villa-Ortega, ${ }^{132}$ J.-Y. Vinet, ${ }^{76}$ S. Vitale, ${ }^{53}$ T. Vo, ${ }^{45}$ H. Vocca, ${ }^{59,58}$ C. Vorvick, ${ }^{51}$ S. P. Vyatchanin, ${ }^{71}$ A. R. Wade, ${ }^{9}$ L. E. Wade, ${ }^{195}$ M. Wade, ${ }^{195}$ R. C. Walet,${ }^{41}$ M. Walker, ${ }^{7}$ G. S. Wallace, ${ }^{26}$ L. Wallace, ${ }^{1}$ S. Walsh, ${ }^{25}$ J. Z. Wang, ${ }^{158}$ S. Wang, ${ }^{23}$ W. H. Wang, ${ }^{125}$ Y. F. Wang, ${ }^{104}$ R. L. Ward, ${ }^{9}$ J. Warner, ${ }^{51}$ M. Was, ${ }^{39}$ N. Y. Washington, ${ }^{1}$ J. Watchi, ${ }^{120}$ B. Weaver, ${ }^{51}$ L. Wei, ${ }^{10,11}$ M. Weinert, ${ }^{10,11}$ A. J. Weinstein, ${ }^{1}$ R. Weiss, ${ }^{53}$ F. Wellmann, ${ }^{10,11}$ L. Wen, ${ }^{78}$ P. Weßels ${ }^{10,11}$ J. W. Westhouse, ${ }^{29}$ K. Wette, ${ }^{9}$ J. T. Whelan, ${ }^{135}$ D. D. White ${ }^{31}$ L. V. White, ${ }^{45}$ B. F. Whiting, ${ }^{34}$ C. Whittle ${ }^{53}$ D. M. Wilken, ${ }^{10,11}$ D. Williams, ${ }^{55}$ M. J. Williams, ${ }^{55}$ A. R. Williamson, ${ }^{141}$ J. L. Willis, ${ }^{1}$ B. Willke, ${ }^{10,11}$ D. J. Wilson, ${ }^{116}$ M. H. Wimmer, ${ }^{10,11}$ W. Winkler, ${ }^{10,11}$ C. C. Wipf, ${ }^{1}$ G. Woan, ${ }^{55}$ J. Woehler,,${ }^{10,11}$ J. K. Wofford, ${ }^{135}$ I. C. F. Wong, ${ }^{104}$ J. Wrangel, ${ }^{10,11}$ J. L. Wright, ${ }^{55}$ D. S. Wu, ${ }^{10,11}$ D. M. Wysocki, ${ }^{135}$ L. Xiao, ${ }^{1}$ H. Yamamoto, ${ }^{1}$ L. Yang, ${ }^{144}$ Y. Yang, ${ }^{34}$ Z. Yang,${ }^{47}$ M. J. Yap, ${ }^{9}$ D. W. Yeeles, ${ }^{101}$ A. Yoon, ${ }^{7}$ Hang Yu, ${ }^{77}$ Haocun Yu, ${ }^{53}$ S. H. R. Yuen, ${ }^{104}$ A. Zadrożny, ${ }^{175}$ M. Zanolin, ${ }^{29}$ T. Zelenova, ${ }^{33}$ J.-P. Zendri, ${ }^{11}$ M. Zevin, ${ }^{15}$ J. Zhang, ${ }^{78}$ L. Zhang, ${ }^{1}$ R. Zhang, ${ }^{34}$ T. Zhang, ${ }^{14}$ C. Zhao, ${ }^{78}$ G. Zhao, ${ }^{120}$ Y. Zheng, ${ }^{70}$ M. Zhou, ${ }^{15}$ Z. Zhou, ${ }^{15}$ X. J. Zhu, ${ }^{6}$ A. B. Zimmerman, ${ }^{150}$ Y. Zlochower, ${ }^{135}$ M. E. Zucker, ${ }^{1,53}$ and J. Zweizig ${ }^{1}$

\section{(LIGO Scientific Collaboration and Virgo Collaboration)}

${ }^{1}$ LIGO, California Institute of Technology, Pasadena, California 91125, USA

${ }^{2}$ Louisiana State University, Baton Rouge, Louisiana 70803, USA

${ }^{3}$ Inter-University Centre for Astronomy and Astrophysics, Pune 411007, India

${ }^{4}$ Dipartimento di Farmacia, Università di Salerno, I-84084 Fisciano, Salerno, Italy

${ }^{5}$ INFN, Sezione di Napoli, Complesso Universitario di Monte S.Angelo, I-80126 Napoli, Italy

${ }^{6}$ OzGrav, School of Physics and Astronomy, Monash University, Clayton 3800, Victoria, Australia

${ }^{7}$ Christopher Newport University, Newport News, Virginia 23606, USA

${ }^{8}$ LIGO Livingston Observatory, Livingston, Louisiana 70754, USA

${ }^{9}$ OzGrav, Australian National University, Canberra, Australian Capital Territory 0200, Australia

${ }^{10}$ Max Planck Institute for Gravitational Physics (Albert Einstein Institute), D-30167 Hannover, Germany

${ }^{11}$ Leibniz Universität Hannover, D-30167 Hannover, Germany

${ }^{12}$ University of Cambridge, Cambridge CB2 1TN, United Kingdom

${ }^{13}$ Theoretisch-Physikalisches Institut, Friedrich-Schiller-Universität Jena, D-07743 Jena, Germany

${ }^{14}$ University of Birmingham, Birmingham B15 2TT, United Kingdom

${ }^{15}$ Center for Interdisciplinary Exploration and Research in Astrophysics (CIERA), Northwestern University, Evanston, Illinois 60208, USA

${ }^{16}$ Instituto Nacional de Pesquisas Espaciais, 12227-010 São José dos Campos, São Paulo, Brazil

${ }^{17}$ Gran Sasso Science Institute (GSSI), I-67100L'Aquila, Italy

${ }^{18}$ INFN, Laboratori Nazionali del Gran Sasso, I-67100 Assergi, Italy

${ }^{19}$ INFN, Sezione di Pisa, I-56127 Pisa, Italy

${ }^{20}$ Università di Pisa, I-56127 Pisa, Italy

${ }^{21}$ International Centre for Theoretical Sciences, Tata Institute of Fundamental Research, Bengaluru 560089, India

${ }^{22}$ University College Dublin, Dublin 4, Ireland

${ }^{23}$ NCSA, University of Illinois at Urbana-Champaign, Urbana, Illinois 61801, USA 
${ }^{24}$ Université de Lyon, Université Claude Bernard Lyon 1, CNRS, Institut Lumière Matière, F-69622 Villeurbanne, France

${ }^{25}$ University of Wisconsin-Milwaukee, Milwaukee, Wisconsin 53201, USA

${ }^{26}$ SUPA, University of Strathclyde, Glasgow G1 1XQ, United Kingdom

${ }^{27}$ Dipartimento di Matematica e Informatica, Università di Udine, I-33100 Udine, Italy

${ }^{28}$ INFN, Sezione di Trieste, I-34127 Trieste, Italy

${ }^{29}$ Embry-Riddle Aeronautical University, Prescott, Arizona 86301, USA

${ }^{30}$ Université de Paris, CNRS, Astroparticule et Cosmologie, F-75013 Paris, France

${ }^{31}$ California State University Fullerton, Fullerton, California 92831, USA

${ }^{32}$ Université Paris-Saclay, CNRS/IN2P3, IJCLab, 91405 Orsay, France

${ }^{33}$ European Gravitational Observatory (EGO), I-56021 Cascina, Pisa, Italy

${ }^{34}$ University of Florida, Gainesville, Florida 32611, USA

${ }^{35}$ Chennai Mathematical Institute, Chennai 603103, India

${ }^{36}$ Columbia University, New York, New York 10027, USA

${ }^{37}$ INFN, Sezione di Roma Tor Vergata, I-00133 Roma, Italy

${ }^{38}$ INFN, Sezione di Roma, I-00185 Roma, Italy

${ }^{39}$ Laboratoire d'Annecy de Physique des Particules (LAPP), Université Grenoble Alpes, Université Savoie Mont Blanc, CNRS/IN2P3, F-74941 Annecy, France

${ }^{40}$ Montclair State University, Montclair, New Jersey 07043, USA

${ }^{41}$ Nikhef, Science Park 105, 1098 XG Amsterdam, Netherlands

${ }^{42}$ Korea Institute of Science and Technology Information, Daejeon 34141, South Korea

${ }^{43}$ INFN Sezione di Torino, I-10125 Torino, Italy

${ }^{44}$ University of Oregon, Eugene, Oregon 97403, USA

${ }^{45}$ Syracuse University, Syracuse, New York 13244, USA

${ }^{46}$ Université de Liège, B-4000 Liège, Belgium

${ }^{47}$ University of Minnesota, Minneapolis, Minnesota 55455, USA

${ }^{48}$ Università degli Studi di Milano-Bicocca, I-20126 Milano, Italy

${ }^{49}$ INFN, Sezione di Milano-Bicocca, I-20126 Milano, Italy

${ }^{50}$ INAF, Osservatorio Astronomico di Brera sede di Merate, I-23807 Merate, Lecco, Italy

${ }^{51}$ LIGO Hanford Observatory, Richland, Washington 99352, USA

${ }^{52}$ Institut de Ciències del Cosmos, Universitat de Barcelona,

C/Martí i Franquès 1, Barcelona, 08028, Spain

${ }^{53}$ LIGO, Massachusetts Institute of Technology, Cambridge, Massachusetts 02139, USA

${ }^{54}$ Dipartimento di Medicina, Chirurgia e Odontoiatria "Scuola Medica Salernitana," Università di Salerno, I-84081 Baronissi, Salerno, Italy

${ }^{55}$ SUPA, University of Glasgow, Glasgow G12 8QQ, United Kingdom

${ }^{56}$ Wigner RCP, RMKI, H-1121 Budapest, Konkoly Thege Miklós út 29-33, Hungary

${ }^{57}$ Stanford University, Stanford, California 94305, USA

${ }^{58}$ INFN, Sezione di Perugia, I-06123 Perugia, Italy

${ }^{59}$ Università di Perugia, I-06123 Perugia, Italy

${ }^{60}$ Università di Padova, Dipartimento di Fisica e Astronomia, I-35131 Padova, Italy

${ }^{61}$ INFN, Sezione di Padova, I-35131 Padova, Italy

${ }^{62}$ Bard College, 30 Campus Road, Annandale-On-Hudson, New York 12504, USA

${ }^{63}$ Montana State University, Bozeman, Montana 59717, USA

${ }^{64}$ Institute for Plasma Research, Bhat, Gandhinagar 382428, India

${ }^{65}$ Nicolaus Copernicus Astronomical Center, Polish Academy of Sciences, 00-716, Warsaw, Poland

${ }^{66}$ OzGrav, University of Adelaide, Adelaide, South Australia 5005, Australia

${ }^{67}$ California State University,

Los Angeles, 5151 State University Drive, Los Angeles, California 90032, USA

${ }^{68}$ INFN, Sezione di Genova, I-16146 Genova, Italy

${ }^{69}$ RRCAT, Indore, Madhya Pradesh 452013, India

${ }^{70}$ Missouri University of Science and Technology, Rolla, Missouri 65409, USA

${ }^{71}$ Faculty of Physics, Lomonosov Moscow State University, Moscow 119991, Russia

${ }^{72}$ SUPA, University of the West of Scotland, Paisley PA1 2BE, United Kingdom

${ }^{73}$ Bar-Ilan University, Ramat Gan, 5290002, Israel

${ }^{74}$ Università degli Studi di Urbino "Carlo Bo," I-61029 Urbino, Italy

${ }^{75}$ INFN, Sezione di Firenze, I-50019 Sesto Fiorentino, Firenze, Italy

${ }^{76}$ Artemis, Université Côte d'Azur, Observatoire Côte d'Azur, CNRS, F-06304 Nice, France

${ }^{77}$ Caltech CaRT, Pasadena, California 91125, USA

${ }^{78}$ OzGrav, University of Western Australia, Crawley, Western Australia 6009, Australia 
${ }^{79}$ Institut de Física d'Altes Energies (IFAE), Barcelona Institute of Science and Technology, and ICREA, E-08193 Barcelona, Spain

${ }^{80}$ Dipartimento di Fisica “E. R. Caianiello," Università di Salerno, I-84084 Fisciano, Salerno, Italy

${ }^{81}$ INFN, Sezione di Napoli, Gruppo Collegato di Salerno, Complesso Universitario di Monte S. Angelo, I-80126 Napoli, Italy

${ }^{82}$ Physik-Institut, University of Zurich, Winterthurerstrasse 190, 8057 Zurich, Switzerland

${ }^{83}$ Università di Roma “La Sapienza," I-00185 Roma, Italy

${ }^{84}$ Université Rennes, CNRS, Institut FOTON-UMR6082, F-3500 Rennes, France

${ }^{85}$ Laboratoire Kastler Brossel, Sorbonne Université, CNRS, ENS-Université PSL, Collège de France, F-75005 Paris, France

${ }^{86}$ Université catholique de Louvain, B-1348 Louvain-la-Neuve, Belgium

${ }^{87}$ Astronomical Observatory Warsaw University, 00-478 Warsaw, Poland

${ }^{88}$ VU University Amsterdam, $1081 \mathrm{HV}$ Amsterdam, Netherlands

${ }^{89}$ Max Planck Institute for Gravitational Physics (Albert Einstein Institute), D-14476 Potsdam-Golm, Germany

${ }^{90}$ University of Maryland, College Park, Maryland 20742, USA

${ }^{91}$ School of Physics, Georgia Institute of Technology, Atlanta, Georgia 30332, USA

${ }^{92}$ Villanova University, 800 Lancaster Avenue, Villanova, Pennsylvania 19085, USA

${ }^{93}$ Center for Computational Astrophysics, Flatiron Institute, New York, New York 10010, USA

${ }^{94}$ Università di Napoli "Federico II," Complesso Universitario di Monte S.Angelo, I-80126 Napoli, Italy

${ }^{95}$ NASA Goddard Space Flight Center, Greenbelt, Maryland 20771, USA

${ }^{96}$ Dipartimento di Fisica, Università degli Studi di Genova, I-16146 Genova, Italy

${ }^{97}$ RESCEU, University of Tokyo, Tokyo, 113-0033, Japan

${ }^{98}$ Tsinghua University, Beijing 100084, China

${ }^{99}$ Università degli Studi di Sassari, I-07100 Sassari, Italy

${ }^{100}$ INFN, Laboratori Nazionali del Sud, I-95125 Catania, Italy

${ }^{101}$ Gravity Exploration Institute, Cardiff University, Cardiff CF24 3AA, United Kingdom

${ }^{102}$ Università di Roma Tor Vergata, I-00133 Roma, Italy

${ }^{103}$ Departamento de Astronomía y Astrofísica, Universitat de València, E-46100 Burjassot, València, Spain

${ }^{104}$ The Chinese University of Hong Kong, Shatin, NT, Hong Kong

${ }^{105}$ Indian Institute of Technology Bombay, Powai, Mumbai 400 076, India

${ }^{106}$ National Tsing Hua University, Hsinchu City, 30013 Taiwan, Republic of China

${ }^{107}$ Charles Sturt University, Wagga Wagga, New South Wales 2678, Australia

${ }^{108}$ OzGrav, Swinburne University of Technology, Hawthorn Victoria 3122, Australia

${ }^{109}$ University of Chicago, Chicago, Illinois 60637, USA

${ }^{110}$ Dipartimento di Ingegneria Industriale (DIIN), Università di Salerno, I-84084 Fisciano, Salerno, Italy

${ }^{111}$ Institut de Physique des 2 Infinis de Lyon, CNRS/IN2P3, Université de Lyon,

Université Claude Bernard Lyon 1, F-69622 Villeurbanne, France

${ }^{112}$ Seoul National University, Seoul 08826, South Korea

${ }^{113}$ Pusan National University, Busan 46241, South Korea

${ }^{114}$ King's College London, University of London, London WC2R 2LS, United Kingdom

${ }^{115}$ INAF, Osservatorio Astronomico di Padova, I-35122 Padova, Italy

${ }^{116}$ University of Arizona, Tucson, Arizona 85721, USA

${ }^{117}$ Rutherford Appleton Laboratory, Didcot OX11 ODE, United Kingdom

${ }^{118}$ OzGrav, University of Melbourne, Parkville, Victoria 3010, Australia

${ }^{119}$ Universitat de les Illes Balears, IAC3-IEEC, E-07122 Palma de Mallorca, Spain

${ }^{120}$ Université Libre de Bruxelles, Brussels 1050, Belgium

${ }^{121}$ Departamento de Matemáticas, Universitat de València, E-46100 Burjassot, València, Spain

${ }^{122}$ Texas Tech University, Lubbock, Texas 79409, USA

${ }^{123}$ The Pennsylvania State University, University Park, Pennsylvania 16802, USA

${ }^{124}$ University of Rhode Island, Kingston, Rhode Island 02881, USA

${ }^{125}$ The University of Texas Rio Grande Valley, Brownsville, Texas 78520, USA

${ }^{126}$ Bellevue College, Bellevue, Washington 98007, USA

${ }^{127}$ Scuola Normale Superiore, Piazza dei Cavalieri, 7-56126 Pisa, Italy

${ }^{128}$ MTA-ELTE Astrophysics Research Group, Institute of Physics, Eötvös University, Budapest 1117, Hungary

${ }^{129}$ University of Szeged, Dóm tér 9, Szeged 6720, Hungary

${ }^{130}$ Maastricht University, 6200 MD, Maastricht, Netherlands

${ }^{131}$ Universität Hamburg, D-22761 Hamburg, Germany 
${ }^{132}$ IGFAE, Campus Sur, Universidade de Santiago de Compostela, 15782 Spain

${ }^{133}$ The University of Sheffield, Sheffield S10 2TN, United Kingdom

${ }^{134}$ Laboratoire des Matériaux Avancés (LMA), Institut de Physique des 2 Infinis de Lyon, CNRS/IN2P3, Université de Lyon, F-69622 Villeurbanne, France

${ }^{135}$ Rochester Institute of Technology, Rochester, New York 14623, USA

${ }^{136}$ Dipartimento di Scienze Matematiche, Fisiche e Informatiche, Università di Parma, I-43124 Parma, Italy

${ }^{137}$ INFN, Sezione di Milano Bicocca, Gruppo Collegato di Parma, I-43124 Parma, Italy

${ }^{138}$ Dipartimento di Ingegneria, Università del Sannio, I-82100 Benevento, Italy

${ }^{139}$ Università di Trento, Dipartimento di Fisica, I-38123 Povo, Trento, Italy

${ }^{140}$ INFN, Trento Institute for Fundamental Physics and Applications, I-38123 Povo, Trento, Italy

${ }^{141}$ University of Portsmouth, Portsmouth, PO1 3FX, United Kingdom

${ }^{142}$ West Virginia University, Morgantown, West Virginia 26506, USA

${ }^{143}$ Stony Brook University, Stony Brook, New York 11794, USA

${ }^{144}$ Colorado State University, Fort Collins, Colorado 80523, USA

${ }^{145}$ Institute for Nuclear Research, Hungarian Academy of Sciences, Bem t'er 18/c, H-4026 Debrecen, Hungary

${ }^{146}$ CNR-SPIN, clo Università di Salerno, I-84084 Fisciano, Salerno, Italy

${ }^{147}$ Scuola di Ingegneria, Università della Basilicata, I-85100 Potenza, Italy

${ }^{148}$ National Astronomical Observatory of Japan, 2-21-1 Osawa, Mitaka, Tokyo 181-8588, Japan

${ }^{149}$ Observatori Astronòmic, Universitat de València, E-46980 Paterna, València, Spain

${ }^{150}$ Department of Physics, University of Texas, Austin, Texas 78712, USA

${ }^{151}$ GRAPPA, Anton Pannekoek Institute for Astronomy and Institute for High-Energy Physics, University of Amsterdam, Science Park 904, 1098 XH Amsterdam, Netherlands

${ }^{152}$ Delta Institute for Theoretical Physics, Science Park 904, 1090 GL Amsterdam, Netherlands

${ }^{153}$ Lorentz, Institute, Leiden University, Niels Bohrweg 2, 2333 CA Leiden, Netherlands

${ }^{154}$ University of British Columbia, Vancouver, British Columbia V6T 1Z4, Canada

${ }^{155}$ Tata Institute of Fundamental Research, Mumbai 400005, India

${ }^{156}$ INAF, Osservatorio Astronomico di Capodimonte, I-80131 Napoli, Italy

${ }^{157}$ Department of Physics, Utrecht University, Princetonplein 1, 3584 CC Utrecht, Netherlands

${ }^{158}$ University of Michigan, Ann Arbor, Michigan 48109, USA

${ }^{159}$ American University, Washington, D.C. 20016, USA

${ }^{160}$ Carleton College, Northfield, Minnesota 55057, USA

${ }^{161}$ University of California, Berkeley, California 94720, USA

${ }^{162}$ Directorate of Construction, Services and Estate Management, Mumbai 400094 India

${ }^{163}$ Universiteit Antwerpen, Prinsstraat 13, 2000 Antwerpen, Belgium

${ }^{164}$ University of Bialystok, 15-424 Bialystok, Poland

${ }^{165}$ University of Southampton, Southampton SO17 1BJ, United Kingdom

${ }^{166}$ University of Washington Bothell, Bothell, Washington 98011, USA

${ }^{167}$ Institute of Applied Physics, Nizhny Novgorod, 603950, Russia

${ }^{168}$ Ewha Womans University, Seoul 03760, South Korea

${ }^{169}$ Inje University Gimhae, South Gyeongsang 50834, South Korea

${ }^{170}$ Korea Astronomy and Space Science Institute, Daejeon 34055, South Korea

${ }^{171}$ National Institute for Mathematical Sciences, Daejeon 34047, South Korea

${ }^{172}$ Ulsan National Institute of Science and Technology, Ulsan 44919, South Korea

${ }^{173}$ Maastricht University, P.O. Box 616, 6200 MD Maastricht, Netherlands

${ }^{174}$ Institute of Mathematics, Polish Academy of Sciences, 00656 Warsaw, Poland

${ }^{175}$ National Center for Nuclear Research, 05-400 Świerk-Otwock, Poland

${ }^{176}$ Cornell University, Ithaca, New York 14850, USA

${ }^{177}$ Université de Montréal/Polytechnique, Montreal, Quebec H3T 1J4, Canada

${ }^{178}$ Laboratoire Lagrange, Université Côte d'Azur, Observatoire Côte d'Azur, CNRS, F-06304 Nice, France

${ }^{179}$ Institute for High-Energy Physics, University of Amsterdam, Science Park 904, 1098 XH Amsterdam, Netherlands

${ }^{180}$ NASA Marshall Space Flight Center, Huntsville, Alabama 35811, USA

${ }^{181}$ University of Washington, Seattle, Washington 98195, USA

${ }^{182}$ Dipartimento di Matematica e Fisica, Università degli Studi Roma Tre, I-00146 Roma, Italy

${ }^{183}$ INFN, Sezione di Roma Tre, I-00146 Roma, Italy

${ }^{184}$ ESPCI, CNRS, F-75005 Paris, France

${ }^{185}$ Università di Camerino, Dipartimento di Fisica, I-62032 Camerino, Italy 
${ }^{186}$ Southern University and A\&M College, Baton Rouge, Louisiana 70813, USA

${ }^{187}$ Dipartimento di Fisica, Università di Trieste, I-34127 Trieste, Italy

${ }^{188}$ Centre Scientifique de Monaco, 8 quai Antoine Ier, MC-98000, Monaco

${ }^{189}$ Indian Institute of Technology Madras, Chennai 600036, India

${ }^{190}$ Institut Pluridisciplinaire Hubert CURIEN, 23 rue du loess, B.P. 28, 67037 Strasbourg cedex 2, France

${ }^{191}$ Museo Storico della Fisica e Centro Studi e Ricerche "Enrico Fermi," I-00184 Roma, Italy

${ }^{192}$ Institut des Hautes Etudes Scientifiques, F-91440 Bures-sur-Yvette, France

${ }^{193}$ Indian Institute of Science Education and Research, Kolkata, Mohanpur, West Bengal 741252, India

${ }^{194}$ Department of Astrophysics/IMAPP, Radboud University Nijmegen,

P.O. Box 9010, 6500 GL Nijmegen, Netherlands

${ }^{195}$ Kenyon College, Gambier, Ohio 43022, USA

${ }^{196}$ Whitman College, 345 Boyer Avenue, Walla Walla, Washington 99362, USA

${ }^{197}$ Consiglio Nazionale delle Ricerche-Istituto dei Sistemi Complessi,

Piazzale Aldo Moro 5, I-00185 Roma, Italy

${ }^{198}$ Hobart and William Smith Colleges, Geneva, New York 14456, USA

${ }^{199}$ Lancaster University, Lancaster LA1 4YW, United Kingdom

${ }^{200}$ Università di Trento, Dipartimento di Matematica, I-38123 Povo, Trento, Italy

${ }^{201}$ Trinity University, San Antonio, Texas 78212, USA

${ }^{202}$ Indian Institute of Science Education and Research, Pune, Maharashtra 411008, India

${ }^{203}$ Dipartimento di Fisica, Università degli Studi di Torino, I-10125 Torino, Italy

${ }^{204}$ Indian Institute of Technology, Palaj, Gandhinagar, Gujarat 382355, India

${ }^{205}$ Centro de Astrofísica e Gravitação (CENTRA), Departamento de Física, Instituto Superior Técnico, Universidade de Lisboa, 1049-001 Lisboa, Portugal

${ }^{206}$ Marquette University, 11420 West Clybourn Street, Milwaukee, Wisconsin 53233, USA

${ }^{207}$ Indian Institute of Technology Hyderabad, Sangareddy, Khandi, Telangana 502285, India

${ }^{208}$ INAF, Osservatorio di Astrofisica e Scienza dello Spazio, I-40129 Bologna, Italy

${ }^{209}$ International Institute of Physics, Universidade Federal do Rio Grande do Norte, Natal RN 59078-970, Brazil

${ }^{210}$ Andrews University, Berrien Springs, Michigan 49104, USA

${ }^{211}$ Van Swinderen Institute for Particle Physics and Gravity, University of Groningen, Nijenborgh 4, 9747 AG Groningen, Netherlands

${ }^{212}$ Concordia University Wisconsin, Mequon, Wisconsin 53097, USA 